Supporting Information for

\title{
a-Amidino Rhodium Carbenes: Key Intermediates for the Preparation of $(E)$-2-Aminomethylene-3-oxoindoles and Pyranoindoles
}

Jing Qian, Guorong Sheng, Kai Huang, Shaojie Liu, Ping Lu*, and Yanguang Wang* Department of Chemistry, Zhejiang University, Hangzhou 310027, P. R. China pinglu@,zju.edu.cn; orgwyg@,zju.edu.cn

\section{Contents}

$\begin{array}{ll}\text { General Information } & \text { S2 }\end{array}$

$\begin{array}{ll}\text { References } & \text { S2 }\end{array}$

Table S1 Optimization of the reaction conditions $\quad$ S3

General Procedure for the Synthesis of $3 \quad$ S4

General Procedure for the Synthesis of $5 \quad$ S4

General Procedure for the Synthesis of $6 \quad$ S4

The ORTEP diagram and Crystal Parameters of 3t S5

The ORTEP diagram and Crystal Parameters of 3o S6

The ORTEP diagram and Crystal Parameters of 5a 4

$\begin{array}{ll}\text { Analysis Data of Products } & \text { S8 }\end{array}$

$\begin{array}{ll}\text { Copies of NMR Spectra } & \text { S22 }\end{array}$ 


\section{General Information}

${ }^{1} \mathrm{H}$ NMR spectra were obtained on 600 or $400 \mathrm{MHz}$ in $\mathrm{CDCl}_{3}$. The chemical shifts were quoted in parts per million (ppm) referenced to $0.0 \mathrm{ppm}$ for tetramethylsilane (TMS) as an internal standard. ${ }^{13} \mathrm{C}$ NMR spectra were recorded on 150 or $100 \mathrm{MHz}$ in $\mathrm{CDCl}_{3}$. The chemical shifts were reported in ppm referenced to the internal solvent signals (77.00 ppm for $\mathrm{CDCl}_{3}$ ). The following abbreviations were used to describe peak patterns where appropriate: $\mathrm{b}=$ broad, $\mathrm{s}=$ singlet, $\mathrm{d}=$ doublet, $\mathrm{t}=$ triplet, $\mathrm{q}=$ quartet, $\mathrm{m}=$ multiplet. Coupling constants $J$ were reported in hertz unit (Hz). Infrared spectra were obtained on an FTIR spectrometer. High-resolution mass spectra (HRMS) data were obtained by using EI, MALDI-TOF, or ESI ionization. Melting points were measured with SGW X-4 micro melting point apparatus. Flash column chromatography was performed employing 300-400 mesh silica gel. Thin layer chromatography (TLC) was performed on silica gel HSGF254.

1,2-Dichloroethane was dried by distillation over $\mathrm{CaH}_{2}$. Toluene was distilled from $\mathrm{Na}$. Chloroform was distilled from phosphorous pentoxide. $\mathrm{Rh}_{2}(\mathrm{Oct})_{4}$, $\mathrm{Rh}_{2}(\mathrm{TFA})_{4}, \mathrm{Rh}_{2}(\mathrm{HFB})_{4}, \mathrm{Rh}_{2}(\mathrm{OAc})_{4}, \mathrm{Cu}(\mathrm{OTf})_{2}, \mathrm{AgOTf}, \mathrm{Rh}_{2}(\mathrm{~S}-\mathrm{DOSP})_{4}, \mathrm{Rh}_{2}(\mathrm{esp})_{2}$, aromatic aldehyde, $\alpha, \beta$-unsaturated aldehyde, $\mathrm{NaH}$ were used as received from the commercial sources. The 3-diazoindolin-2-imines and its analogues were prepared according to the published methods. ${ }^{1,2}$

\section{References}

1. Xing, Y. P.; Sheng, G. R.; Wang, J.; Lu, P.; Wang, Y. G. Org. Lett. 2014, 16, 1244.

2. Sheng, G. R.; Huang, K.; Chi, Z. H.; Ding, H. L.; Xing, Y. P.; Lu, P.; Wang, Y. G. Org. Lett. 2014, 16, 5096. 
Table S1 Optimization of Reaction Conditions

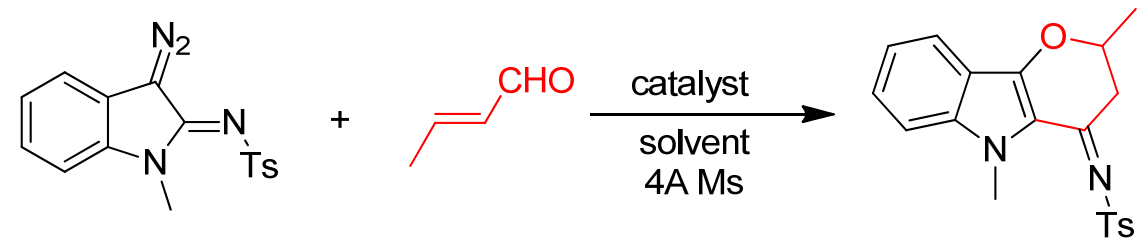

$1 \mathbf{a}$

$4 a$

$5 a$

\begin{tabular}{|c|c|c|c|c|c|}
\hline entry & catalyst & temp $\left({ }^{\circ} \mathrm{C}\right)$ & time (h) & solvent & yields ${ }^{\mathrm{b}}(\%)$ \\
\hline 1 & $\mathrm{Rh}_{2}(\mathrm{TFA})_{4}$ & 60 & 3 & Toluene & 34 \\
\hline 2 & $\mathrm{Rh}_{2}(\mathrm{HFD})_{4}$ & 60 & 3 & Toluene & trace \\
\hline 3 & $\mathrm{Rh}_{2}(\mathrm{~s}-\mathrm{DOSP})_{4}$ & 60 & 3 & Toluene & trace \\
\hline 4 & $\mathrm{Rh}_{2}(\mathrm{OAc})_{4}$ & 60 & 3 & Toluene & 18 \\
\hline 5 & $\mathrm{Rh}_{2}(\mathrm{Oct})_{4}$ & 60 & 3 & Toluene & trace \\
\hline 6 & $\mathrm{Rh}_{2}(\mathrm{esp})_{4}$ & 60 & 3 & Toluene & trace \\
\hline 7 & $\mathrm{Cu}(\mathrm{OTf})_{2}$ & 60 & 3 & Toluene & trace \\
\hline 8 & AgOTf & 60 & 3 & Toluene & trace \\
\hline 9 & $\mathrm{Rh}_{2}(\mathrm{HFB})_{4}$ & $\mathrm{rt}$ & 3 & Toluene & 21 \\
\hline 10 & $\mathrm{Rh}_{2}(\mathrm{HFB})_{4}$ & 40 & 3 & Toluene & 27 \\
\hline 11 & $\mathrm{Rh}_{2}(\mathrm{HFB})_{4}$ & 120 & 3 & Toluene & 31 \\
\hline 12 & $\mathrm{Rh}_{2}(\mathrm{HFB})_{4}$ & 60 & 2 & Toluene & 29 \\
\hline 13 & $\mathrm{Rh}_{2}(\mathrm{HFB})_{4}$ & 60 & 4 & Toluene & 33 \\
\hline 14 & $\mathrm{Rh}_{2}(\mathrm{HFB})_{4}$ & 60 & 3 & DCE & 25 \\
\hline 15 & $\mathrm{Rh}_{2}(\mathrm{HFB})_{4}$ & 60 & 3 & $\mathrm{CHCl}_{3}$ & 22 \\
\hline 16 & $\mathrm{Rh}_{2}(\mathrm{HFB})_{4}$ & 60 & 3 & DCM & 31 \\
\hline 17 & $\mathrm{Rh}_{2}(\mathrm{HFB})_{4}$ & 60 & 3 & $\mathrm{CH}_{3} \mathrm{CN}$ & trace \\
\hline 18 & $\mathrm{Rh}_{2}(\mathrm{HFB})_{4}$ & 60 & 3 & DMSO & trace \\
\hline 19 & $\mathrm{Rh}_{2}(\mathrm{HFB})_{4}$ & 60 & 3 & Toluene & $19^{c}$ \\
\hline 20 & $\mathrm{Rh}_{2}(\mathrm{HFB})_{4}$ & 60 & 3 & Toluene & $30^{\mathrm{d}}$ \\
\hline
\end{tabular}

${ }^{\mathrm{a}}$ Reaction conditions: $1 \mathrm{a}(0.20 \mathrm{mmol}), \mathbf{4 a}(0.30 \mathrm{mmol})$, catalyst $(0.005 \mathrm{mmol})$, solvent $(2 \mathrm{~mL}), 4 \AA \mathrm{MS}(20 \mathrm{mg}) .{ }^{\mathrm{b}}$ Isolated yields. ${ }^{\mathrm{c}}$ Without $4 \AA \mathrm{MS} .{ }^{\mathrm{d}} \mathbf{4 a}(0.60 \mathrm{mmol})$. 


\section{General Procedure for the Synthesis of 3}

To an oven-dried Heavy-wall Pressure Vessel equipped with a magnetic stirring bar were added 1 (0.2 mmol), 2 (0.9 mmol), $\mathrm{Rh}_{2}(\mathrm{HFB})_{4}(0.005 \mathrm{mmol}), 4 \AA \mathrm{MS}(20 \mathrm{mg})$ and dry DCM $(2 \mathrm{~mL})$ under air atmosphere. Then the mixture was stirred at $100{ }^{\circ} \mathrm{C}$ for $4 \mathrm{~h}$ until the reaction was completed (monitored by TLC). After the reaction mixture was cooled to room temperature, the solvent was removed in vacuum and the residue was purified by column chromatography on silica gel (petroleum ether/ethyl acetate $4: 1$ ) to give pure product 3 .

\section{General Procedure for the Synthesis of 5}

To an oven-dried flask equipped with a magnetic stirring bar were added $\mathbf{1}(0.2 \mathrm{mmol})$, $4(0.3 \mathrm{mmol}), \mathrm{Rh}_{2}(\mathrm{TFA})_{4}(0.005 \mathrm{mmol}), 4 \AA \mathrm{MS}(20 \mathrm{mg})$ and dry toluene $(2 \mathrm{~mL})$ under air atmosphere. Then the mixture was stirred at $60{ }^{\circ} \mathrm{C}$ for $3 \mathrm{~h}$. After the reaction completed, the reaction mixture was cooled to room temperature. The solvent was removed in vacuum and the residue was purified by column chromatography on silica gel (petroleum ether/ethyl acetate $5: 1$ ) to give the product 5 .

\section{General Procedure for the Synthesis of 6}

To an oven-dried flask equipped with a magnetic stirring bar were added $\mathbf{1}(0.2 \mathrm{mmol})$, $\mathrm{NaH}(0.6 \mathrm{mmol})$ and dry THF $(2 \mathrm{~mL})$ under air atmosphere. Then the mixture was stirred at reflux temperature for $12 \mathrm{~h}$. After the reaction completed, the reaction mixture was cooled to room temperature. The solvent was removed in vacuum and the residue was purified by column chromatography on silica gel (petroleum ether/ethyl acetate $4: 1$ ) to give the product 6 . 


\section{The ORTEP diagram and Crystal Parameters of $3 t$}

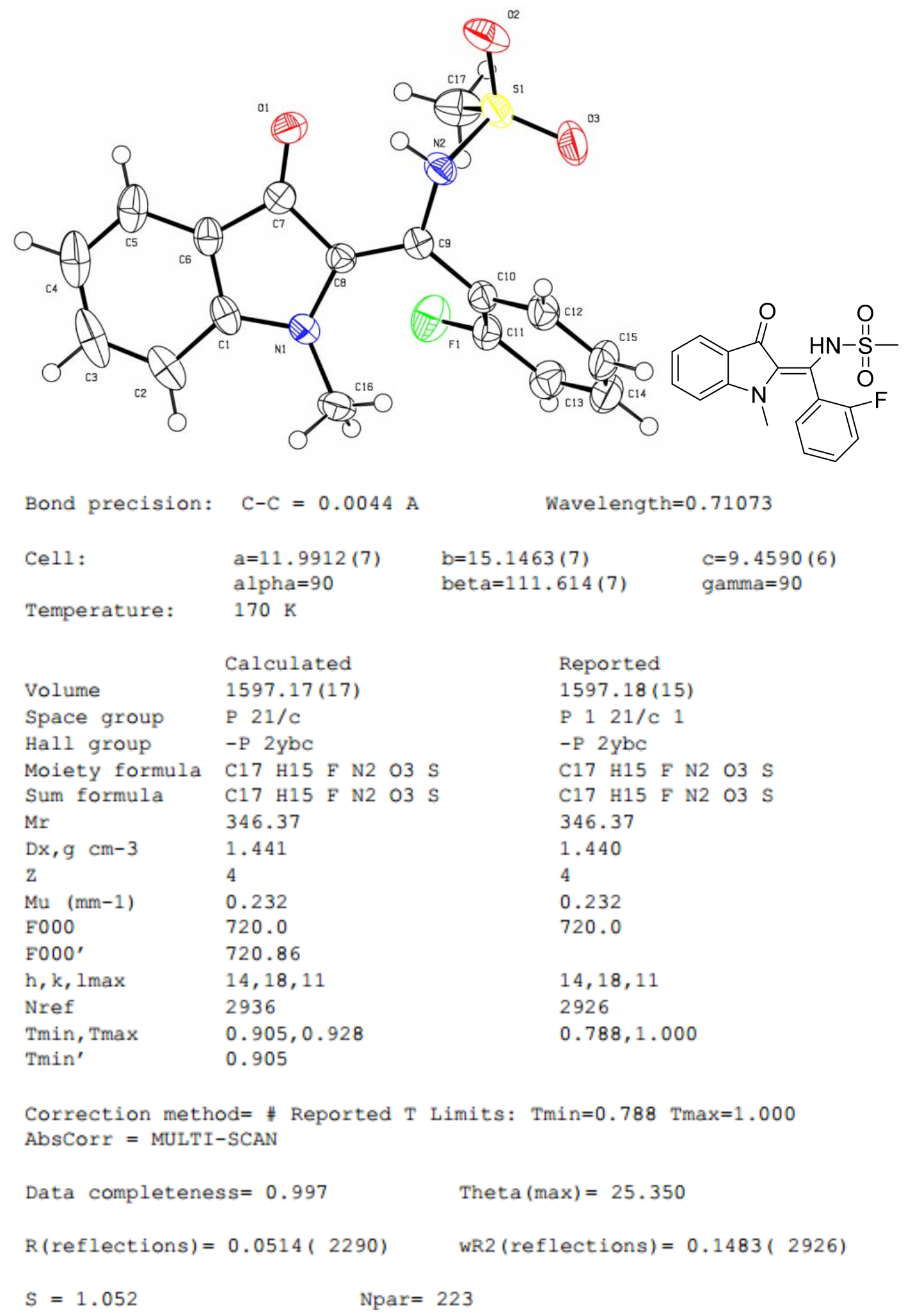




\section{The ORTEP diagram and Crystal Parameters of 30}

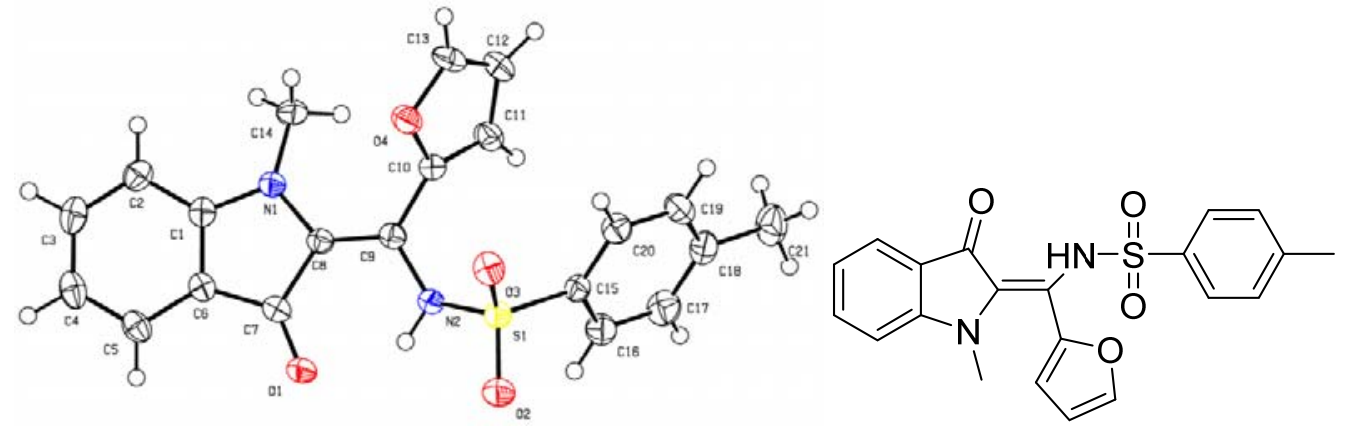

Bond precision: $\quad \mathrm{C}-\mathrm{C}=0.0030 \mathrm{~A}$ Wavelength $=0.71073$
Cell:
$a=7.3603(3)$
$=7.5430(4)$
$C=17.2163(9)$
alpha $=87.384(5)$
Temperature:
$170 \mathrm{~K}$
beta $=86.032(4)$
gamma $=74.912(5)$

\begin{tabular}{|c|c|c|}
\hline & Calculated & Reported \\
\hline Volume & $920.29(8)$ & $920.29(8)$ \\
\hline Space group & P -1 & P -1 \\
\hline Hall group & $-\mathrm{P} 1$ & $-\mathrm{P} 1$ \\
\hline Moiety formula & $\mathrm{C} 21 \mathrm{H} 18 \mathrm{~N} 2 \quad 04 \mathrm{~S}$ & $\mathrm{C} 21$ H18 N2 O4 S \\
\hline Sum formula & $\mathrm{C} 21 \mathrm{H} 18 \mathrm{~N} 204 \mathrm{~S}$ & $\mathrm{C} 21$ H18 N2 O4 $\mathrm{S}$ \\
\hline $\mathrm{Mr}$ & 394.43 & 394.43 \\
\hline $\mathrm{Dx}, \mathrm{g} \mathrm{cm}-3$ & 1.423 & 1.423 \\
\hline 2 & 2 & 2 \\
\hline Mu $(m m-1)$ & 0.207 & 0.207 \\
\hline F000 & 412.0 & 412.0 \\
\hline F000' & 412.44 & \\
\hline $\mathrm{h}, \mathrm{k}, 1 \mathrm{max}$ & $8,9,20$ & $8,9,20$ \\
\hline Nref & 3351 & 3336 \\
\hline $\operatorname{Tmin}, \operatorname{Tmax}$ & $0.917,0.959$ & $0.707,1.000$ \\
\hline Tmin' & 0.917 & \\
\hline \multicolumn{3}{|c|}{$\begin{array}{l}\text { Correction method }=\text { \# Reported T Limits: } \operatorname{Tmin}=0.707 \text { Tmax }=1.000 \\
\text { AbsCorr }=\text { MULTI-SCAN }\end{array}$} \\
\hline \multicolumn{2}{|c|}{ Data completeness $=0.996$} & Theta $(\max )=25.350$ \\
\hline \multicolumn{2}{|c|}{$\mathrm{R}$ (reflections) $=0.0422(2763)$} & WR2 (reflections) $=0.1145(3336)$ \\
\hline$S=1.052$ & Npar $=$ & $=259$ \\
\hline
\end{tabular}




\section{The ORTEP diagram and Crystal Parameters of 5a}
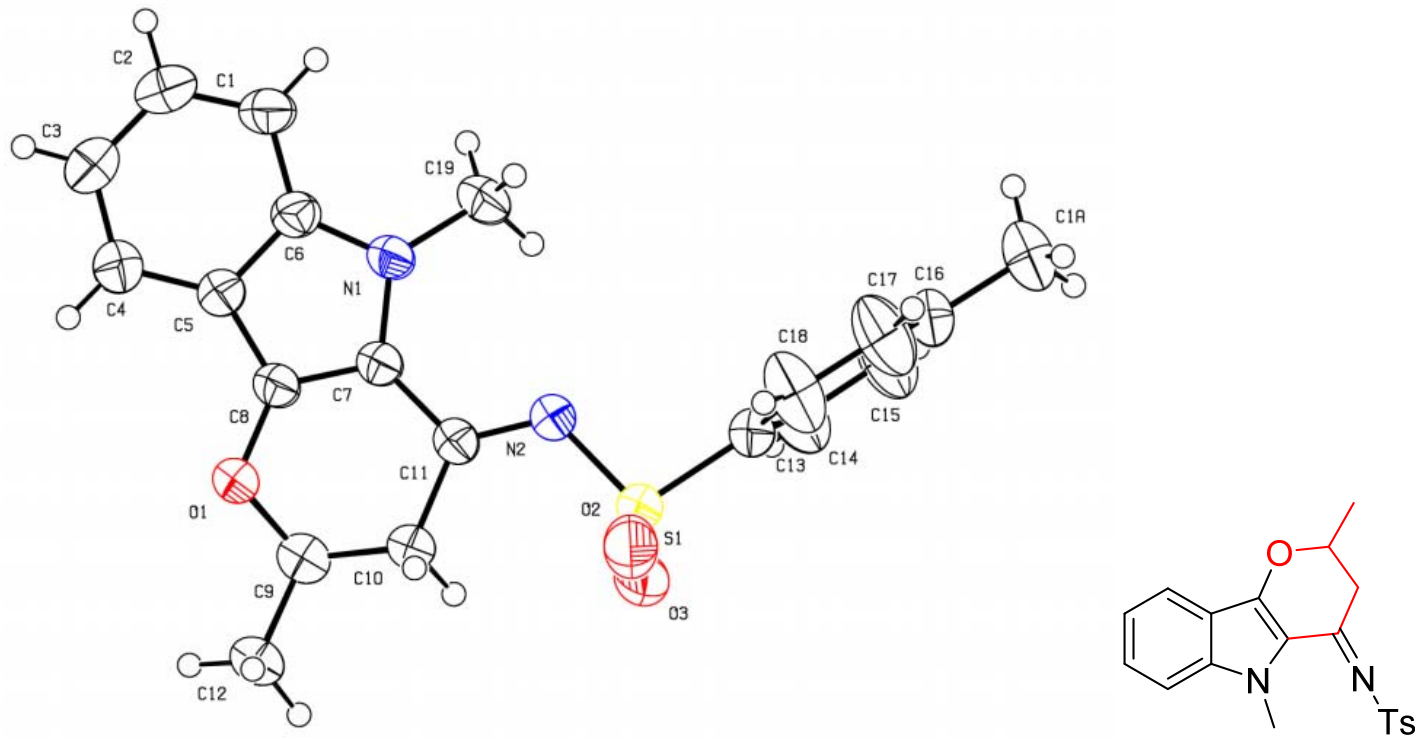

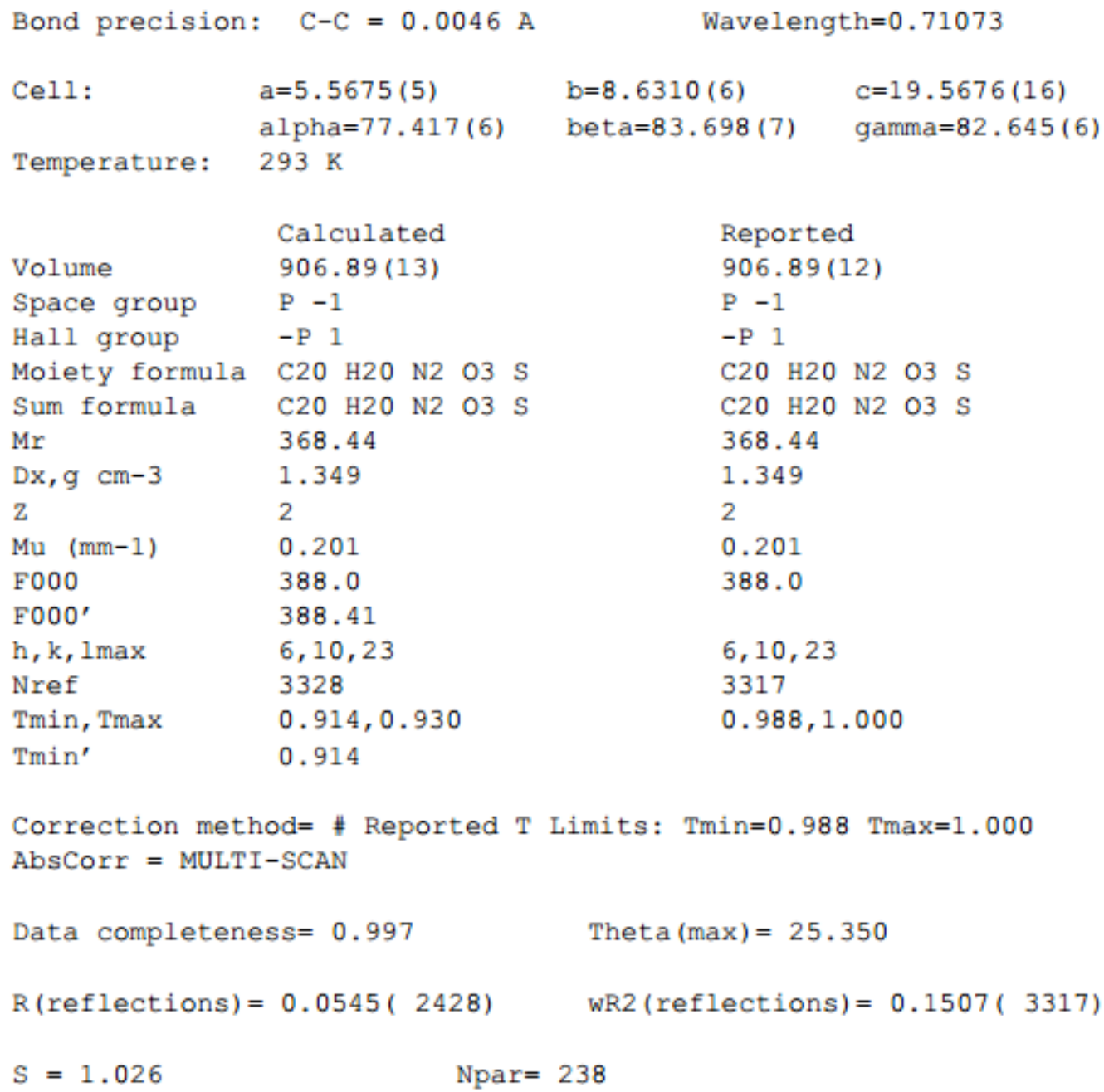




\section{Analysis Data of Products}

(E)- $N$-((2-fluorophenyl)(1-methyl-3-oxoindolin-2-ylidene)methylmethylbenzenesulfonamide (3a):<smiles>Cc1ccc(S(=O)(=O)N/C(=C2/C(=O)c3ccccc3N2C)c2ccccc2F)cc1</smiles>

Red solid (56.5 mg, 67\%); M.p. $164-165{ }^{\circ} \mathrm{C} ;{ }^{1} \mathrm{H}$ NMR (400 MHz, $\mathrm{CDCl}_{3}$ ) $\delta 12.25$ (s, 1H), 7.75 (d, $J$ $=7.7 \mathrm{~Hz}, 1 \mathrm{H}), 7.52-7.42(\mathrm{~m}, 4 \mathrm{H}), 7.29-7.23(\mathrm{~m}, 1 \mathrm{H}), 7.18(\mathrm{dd}, J=11.8,7.8 \mathrm{~Hz}, 3 \mathrm{H}), 7.06(\mathrm{t}, J=8.9$ $\mathrm{Hz}, 1 \mathrm{H}), 6.92(\mathrm{t}, J=7.4 \mathrm{~Hz}, 1 \mathrm{H}), 6.84(\mathrm{~d}, J=8.4 \mathrm{~Hz}, 1 \mathrm{H}), 2.65(\mathrm{~s}, 3 \mathrm{H}), 2.37(\mathrm{~s}, 3 \mathrm{H}),{ }^{13} \mathrm{C}$ NMR $(100$ $\left.\mathrm{MHz}, \mathrm{CDCl}_{3}\right) \delta 184.3,160.3(\mathrm{~d}, J=251.5 \mathrm{~Hz}), 152.9,143.9,136.8,135.7,132.8(\mathrm{~d}, J=2.1 \mathrm{~Hz}), 13$ $2.6(\mathrm{~d}, J=8.2 \mathrm{~Hz}), 129.9,129.4,127.5,125.6,124.6,123.6(\mathrm{~d}, J=3.6 \mathrm{~Hz}), 120.2,119.3$, 118.8 (d, $J=15.2 \mathrm{~Hz}$ ), 115.5 (d, $J=21.1 \mathrm{~Hz}), 109.6$, 32.0 , 21.6 ; IR (film): 3063, 2949, 2924, 2845, 1926, 1 $619,1345,1201,1185,1107 \mathrm{~cm}^{-1}$; HRMS (MALDI-TOF): calcd for $\mathrm{C}_{23} \mathrm{H}_{19} \mathrm{FN}_{2} \mathrm{O}_{3} \mathrm{~S}+\mathrm{H}^{+}\left([\mathrm{M}+\mathrm{H}]^{+}\right)$: 423.1173, Found: 423.1151.

(E)-N-((3-fluorophenyl)(1-methyl-3-oxoindolin-2-ylidene)methyl)-4-methylbenzenesulfonamide (3b):

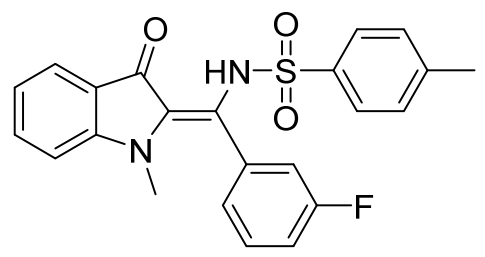

Red solid (52.3 mg, 62\%); M.p. $145-146{ }^{\circ} \mathrm{C} ;{ }^{1} \mathrm{H}$ NMR (400 MHz, $\left.\mathrm{CDCl}_{3}\right) \delta 12.06$ (s, 1H), 7.74 (d, J $=7.6 \mathrm{~Hz}, 1 \mathrm{H}), 7.51-7.32(\mathrm{~m}, 4 \mathrm{H}), 7.16(\mathrm{~d}, J=7.7 \mathrm{~Hz}, 3 \mathrm{H}), 7.09(\mathrm{~d}, J=7.4 \mathrm{~Hz}, 1 \mathrm{H}), 6.98-6.83(\mathrm{~m}$, 3H), $2.62(\mathrm{~s}, 3 \mathrm{H}), 2.37$ (s, 3H); ${ }^{13} \mathrm{C}$ NMR $\left(100 \mathrm{MHz}, \mathrm{CDCl}_{3}\right) \delta 184.5,161.9(\mathrm{~d}, J=248.1 \mathrm{~Hz}), 153.3$, $144.1,136.7,135.7,135.0,132.8(\mathrm{~d}, J=8.1 \mathrm{~Hz}), 129.5,129.5,127.7,127.0(\mathrm{~d}, J=3.1 \mathrm{~Hz}), 125.4$, $124.5,120.5,119.6,118.0(\mathrm{~d}, J=22.8 \mathrm{~Hz}), 117.4(\mathrm{~d}, J=21.0 \mathrm{~Hz}), 110.1,33.6,21.6$; IR (film): 3067, 2921, 1867, 1748, 1620, 1573, 1185, 1086, 899, $716 \mathrm{~cm}^{-1}$; HRMS (MALDI-TOF): calcd for $\mathrm{C}_{23} \mathrm{H}_{19} \mathrm{FN}_{2} \mathrm{O}_{3} \mathrm{~S}+\mathrm{H}^{+}\left([\mathrm{M}+\mathrm{H}]^{+}\right): 423.1173$, Found: 423.1170 .

(E)-N-((4-fluorophenyl)(1-methyl-3-oxoindolin-2-ylidene)methyl)-4-methylbenzenesulfonamide (3c):<smiles>Cc1ccc(S(=O)(=O)NC(=C2C(=O)c3ccccc3N2C)c2ccc(F)cc2)cc1</smiles>

Red solid (51.5 mg, 61\%); M.p. 163-164 ${ }^{0} \mathrm{C} ;{ }^{1} \mathrm{H}$ NMR (400 MHz, $\left.\mathrm{CDCl}_{3}\right) \delta 12.11(\mathrm{~s}, 1 \mathrm{H}), 7.75(\mathrm{~d}, J=$ $7.7 \mathrm{~Hz}, 1 \mathrm{H}), 7.50-7.44(\mathrm{~m}, 1 \mathrm{H}), 7.41(\mathrm{~d}, J=8.0 \mathrm{~Hz}, 2 \mathrm{H}), 7.28-7.22(\mathrm{~m}, 2 \mathrm{H}), 7.15(\mathrm{~d}, J=8.0 \mathrm{~Hz}, 2 \mathrm{H})$, $7.11-7.05$ (m, 2H), 6.94 (t, $J=7.5 \mathrm{~Hz}, 1 \mathrm{H}), 6.87$ (d, $J=8.3 \mathrm{~Hz}, 1 \mathrm{H}), 2.61$ (s, 3H), $2.36(\mathrm{~s}, 3 \mathrm{H}) ;{ }^{13} \mathrm{C}$ 
NMR $\left(100 \mathrm{MHz}, \mathrm{CDCl}_{3}\right) \delta 184.1,163.8(\mathrm{~d}, J=251.9 \mathrm{~Hz}), 153.2,144.0,136.7,136.2,135.5,133.1$ (d, $J=8.6 \mathrm{~Hz}), 129.4,127.6,126.7$ (d, $J=3.4 \mathrm{~Hz}), 126.2$, 124.5 , 120.6 , 119.5 , 115.1 (d, $J=21.9$ Hz), 110.1 , 33.7 , 21.6 ; IR (film): 3065, 2944, 2836, 2795, 1909, 1649, 1505, 1487, 1345, $1085 \mathrm{~cm}^{-1}$; HRMS (ESI): calcd for $\mathrm{C}_{23} \mathrm{H}_{19} \mathrm{FN}_{2} \mathrm{O}_{3} \mathrm{~S}-\mathrm{H}^{+}\left(\mathrm{M}-\mathrm{H}^{+}\right)$: 421.1028, Found: 421.1028 .

(E)-N-((3-bromophenyl)(1-methyl-3-oxoindolin-2-ylidene)methyl)-4-methylbenzenesulfonamide (3d):<smiles>Cc1ccc(S(=O)(=O)N/C(=C2/C(=O)c3ccccc3N2C)c2cccc(Br)c2)cc1</smiles>

Red solid (51.1 mg, 53\%); M.p. $185-186{ }^{0} \mathrm{C} ;{ }^{1} \mathrm{H}$ NMR (400 MHz, $\left.\mathrm{CDCl}_{3}\right) \delta 12.11(\mathrm{~s}, 1 \mathrm{H}), 7.79-7.73(\mathrm{~m}$, 1H), 7.62-7.54 (m, 1H), 7.51-7.45 (m, 1H), $7.42-7.34(\mathrm{~m}, 3 \mathrm{H}), 7.32-7.25(\mathrm{~m}, 1 \mathrm{H}), 7.17(\mathrm{~d}, J=8.1 \mathrm{~Hz}$, 2H), $7.12(\mathrm{t}, J=1.8 \mathrm{~Hz}, 1 \mathrm{H}), 6.94(\mathrm{t}, J=7.4 \mathrm{~Hz}, 1 \mathrm{H}), 6.87(\mathrm{~d}, J=8.4 \mathrm{~Hz}, 1 \mathrm{H}), 2.62(\mathrm{~s}, 3 \mathrm{H}), 2.38(\mathrm{~s}$, $3 \mathrm{H}) ;{ }^{13} \mathrm{C}$ NMR $\left(100 \mathrm{MHz}, \mathrm{CDCl}_{3}\right) \delta 184.6,153.3,144.2,136.7,135.7,134.7,133.4,133.2,132.6$, $129.9,129.5,129.4,127.7,125.3,124.6,121.8,120.5,119.5,110.0,33.7,21.6$; IR (film): 3061, 2942, 2851, 1905, 1649, 1618, 1486, 1342, 1204, $1043 \mathrm{~cm}^{-1}$; HRMS (ESI): calcd for $\mathrm{C}_{23} \mathrm{H}_{19} \mathrm{BrN}_{2} \mathrm{O}_{3} \mathrm{~S}+\mathrm{H}^{+}$ $\left([\mathrm{M}+\mathrm{H}]^{+}\right): 483.0373$, Found: 483.037 .

(E)-N-((3,4-difluorophenyl)(1-methyl-3-oxoindolin-2-ylidene)methyl)-4-methylbenzenesulfonamide (3e):

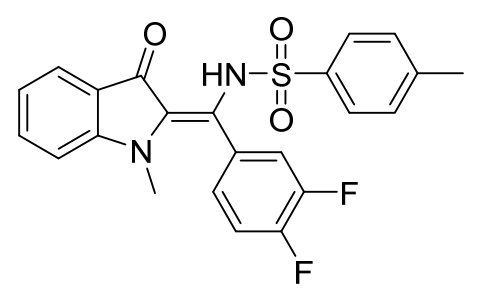

Red solid (48.4 mg, 55\%); M.p. 197-198 ${ }^{\circ} \mathrm{C}$; ${ }^{1} \mathrm{H}$ NMR (400 MHz, $\left.\mathrm{CDCl}_{3}\right) \delta 11.94$ (s, 1H), 7.74 (d, J= $7.7 \mathrm{~Hz}, 1 \mathrm{H}), 7.52-7.42(\mathrm{~m}, 3 \mathrm{H}), 7.24-7.15(\mathrm{~m}, 3 \mathrm{H}), 7.10-7.02(\mathrm{~m}, 2 \mathrm{H}), 6.95(\mathrm{t}, J=7.4 \mathrm{~Hz}, 1 \mathrm{H}), 6.88$ $(\mathrm{d}, J=8.3 \mathrm{~Hz}, 1 \mathrm{H}), 2.65(\mathrm{~s}, 2 \mathrm{H}), 2.37(\mathrm{~s}, 3 \mathrm{H}) ;{ }^{13} \mathrm{C} \mathrm{NMR}\left(100 \mathrm{MHz}, \mathrm{CDCl}_{3}\right) \delta 184.8,153.5,151.4(\mathrm{dd}$, $\left.J_{C-F}=253.0 \mathrm{~Hz}, \mathrm{~J}_{\mathrm{C}-\mathrm{F}}=12.0 \mathrm{~Hz}\right), 149.7\left(\mathrm{dd}, J_{C-F}=250.0 \mathrm{~Hz}, J_{C-F}=13.0 \mathrm{~Hz}\right), 144.3,136.6,135.9,133.6,129.5$ $127.9-127.7$ (m), 127.6, 125.7, 124.6, 120.6, 120.2 (d, $J=18.5 \mathrm{~Hz}), 119.8,117.0(\mathrm{~d}, J=17.7 \mathrm{~Hz})$, 110.1,33.8,21.6; IR(film): 3060, 2930, 2845, 1867, 1747, 1614, 1557, 1486, 1085, $747 \mathrm{~cm}^{-1}$; HRMS (ESI): calcd for $\mathrm{C}_{23} \mathrm{H}_{18} \mathrm{~F}_{2} \mathrm{~N}_{2} \mathrm{O}_{3} \mathrm{~S}+\mathrm{H}^{+}\left([\mathrm{M}+\mathrm{H}]^{+}\right)$: 441.1079 , Found: 441.1083 .

(E)-4-methyl- $N$-((1-methyl-3-oxoindolin-2-ylidene)(2-nitrophenyl)methyl)benzenesulfonamide (3f):<smiles>Cc1ccc(S(=O)(=O)N/C(=C2\C(=O)c3ccccc3N2C)c2ccccc2[N+](=O)[O-])cc1</smiles>

Red solid(26.9 mg, 30\%); M.p. 191-192 ${ }^{0} \mathrm{C} ;{ }^{1} \mathrm{H}$ NMR (400 MHz, $\left.\mathrm{CDCl}_{3}\right) \delta 12.26(\mathrm{~s}, 1 \mathrm{H}), 8.22(\mathrm{~d}, J=$ 
8.1 Hz, 1H), $7.77(\mathrm{~d}, J=7.8 \mathrm{~Hz}, 1 \mathrm{H}), 7.73-7.67(\mathrm{~m}, 1 \mathrm{H}), 7.66-7.59(\mathrm{~m}, 1 \mathrm{H}), 7.50-7.41(\mathrm{~m}, 3 \mathrm{H}), 7.17$ (t, $J=7.7 \mathrm{~Hz}, 3 \mathrm{H}), 6.97(\mathrm{t}, J=7.5 \mathrm{~Hz}, 1 \mathrm{H}), 6.90(\mathrm{~d}, J=8.4 \mathrm{~Hz}, 1 \mathrm{H}), 2.67(\mathrm{~s}, 3 \mathrm{H}), 2.38(\mathrm{~s}, 3 \mathrm{H}) ;{ }^{13} \mathrm{C}$ NMR $\left(100 \mathrm{MHz}, \mathrm{CDCl}_{3}\right) \delta 178.5,150.7,148.3,144.1,139.6,137.2,134.7,133.0,132.6,131.5,129.6$, 127.3, 126.6, 124.7, 124.0, 123.3, 119.7, 119.6, 109.9, 32.4, 21.6; IR (film): 3064, 2924, 1740, 1571, 1530, 1347, 1204, 1202, 883, $789 \mathrm{~cm}^{-1}$; HRMS (ESI): calcd for $\mathrm{C}_{23} \mathrm{H}_{19} \mathrm{~N}_{3} \mathrm{O}_{5} \mathrm{~S}-\mathrm{H}^{+}\left(\mathrm{M}-\mathrm{H}^{+}\right): 448.0973$, Found: 448.0972.

(E)-4-methyl-N-((1-methyl-3-oxoindolin-2-ylidene)(4-nitrophenyl)methyl)benzenesulfonamide (3g):

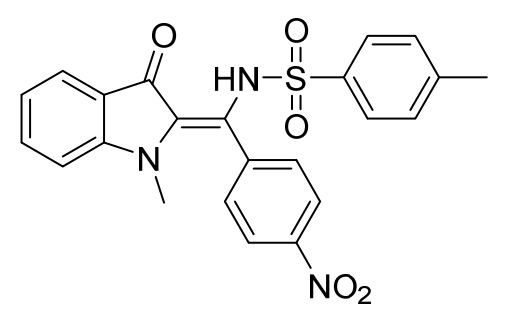

Red solid (44.9 mg, 50\%); M.p. 235-236 ${ }^{\circ} \mathrm{C} ;{ }^{1} \mathrm{H}$ NMR (400 MHz, $\left.\mathrm{CDCl}_{3}\right) \delta 11.79(\mathrm{~s}, 1 \mathrm{H}), 8.26(\mathrm{~d}, J=$ $8.3 \mathrm{~Hz}, 2 \mathrm{H}), 7.74(\mathrm{~d}, J=7.7 \mathrm{~Hz}, 1 \mathrm{H}), 7.56-7.47(\mathrm{~m}, 3 \mathrm{H}), 7.44(\mathrm{~d}, J=8.0 \mathrm{~Hz}, 2 \mathrm{H}), 7.18(\mathrm{~d}, J=8.0 \mathrm{~Hz}$, 2H), $6.97(\mathrm{t}, J=7.5 \mathrm{~Hz}, 1 \mathrm{H}), 6.87(\mathrm{~d}, J=8.3 \mathrm{~Hz}, 1 \mathrm{H}), 2.61(\mathrm{~s}, 3 \mathrm{H}), 2.38(\mathrm{~s}, 3 \mathrm{H}) ;{ }^{13} \mathrm{C}$ NMR $(100 \mathrm{MHz}$, $\left.\mathrm{CDCl}_{3}\right) \delta 185.5,153.8,148.5,144.4,137.9,136.4,136.2,132.1,131.9,129.6,127.6,126.5,124.8$, 122.9, 120.5, 120.1, 110.1, 34.3, 21.6; IR(film): 3104, 3065, 2924, 2857, 1741, 1618, 1520, 1485, 1342 , $1098 \mathrm{~cm}^{-1}$; HRMS (ESI): calcd for $\mathrm{C}_{23} \mathrm{H}_{19} \mathrm{~N}_{3} \mathrm{O}_{5} \mathrm{~S}+\mathrm{H}^{+}\left([\mathrm{M}+\mathrm{H}]^{+}\right)$: 450.1118 , Found: 450.1121.

(E)-4-methyl- $N$-((1-methyl-3-oxoindolin-2-ylidene)(p-tolyl)methyl)benzenesulfonamide (3h):<smiles>Cc1ccc(/C(NS(=O)(=O)c2ccc(C)cc2)=C2\C(=O)c3ccccc3N2C)cc1</smiles>

Red solid (31.8 mg, 38\%); M.p. 206-207 ${ }^{0} \mathrm{C} ;{ }^{1} \mathrm{H}$ NMR (400 MHz, $\left.\mathrm{CDCl}_{3}\right) \delta 12.15$ (s, 1H), $7.79-7.72$ (m, 1H), $7.49-7.39(\mathrm{~m}, 3 \mathrm{H}), 7.22-7.11(\mathrm{~m}, 6 \mathrm{H}), 6.92(\mathrm{t}, J=7.4 \mathrm{~Hz}, 1 \mathrm{H}), 6.86(\mathrm{~d}, J=8.3 \mathrm{~Hz}, 1 \mathrm{H})$, $2.61(\mathrm{~s}, 3 \mathrm{H}), 2.43(\mathrm{~s}, 3 \mathrm{H}), 2.36(\mathrm{~s}, 3 \mathrm{H}) ;{ }^{13} \mathrm{C} \mathrm{NMR}\left(100 \mathrm{MHz}, \mathrm{CDCl}_{3}\right) \delta 183.3,152.8,143.8,140.8$, 138.7, 136.9, 135.07 (s), 130.9, 129.3, 128.5, 127.9, 127.7, 125.1, 124.3, 120.5, 119.2, 110.0, 33.5, 21.7, 21.6; IR (film): 3059, 3024, 2946, 2922, 1913, 1618, 1508, 1436, 1344, $1043 \mathrm{~cm}^{-1}$; HRMS (ESI): calcd for $\mathrm{C}_{24} \mathrm{H}_{22} \mathrm{~N}_{2} \mathrm{O}_{3} \mathrm{~S}-\mathrm{H}^{+}\left(\mathrm{M}-\mathrm{H}^{+}\right)$: 417.1278, Found: 417.1275.

(E)-4-methyl- $N$-((1-methyl-3-oxoindolin-2-ylidene)(phenyl)methyl)benzenesulfonamide (3i):<smiles>Cc1ccc(S(=O)(=O)NC(=C2C(=O)c3ccccc3N2C)c2ccccc2)cc1</smiles> 
Red solid (41.2 mg, 51\%); M.p. 168-169 ${ }^{\circ} \mathrm{C} ;{ }^{1} \mathrm{H}$ NMR (400 MHz, $\left.\mathrm{CDCl}_{3}\right) \delta 12.23(\mathrm{~s}, 1 \mathrm{H}), 7.75(\mathrm{~d}, J=$ $7.7 \mathrm{~Hz}, 1 \mathrm{H}), 7.51-7.43(\mathrm{~m}, 2 \mathrm{H}), 7.42-7.33(\mathrm{~m}, 4 \mathrm{H}), 7.24(\mathrm{~d}, J=7.5 \mathrm{~Hz}, 2 \mathrm{H}), 7.13(\mathrm{~d}, J=7.9 \mathrm{~Hz}, 2 \mathrm{H})$, $6.92(\mathrm{t}, J=7.4 \mathrm{~Hz}, 1 \mathrm{H}), 6.86(\mathrm{~d}, J=8.3 \mathrm{~Hz}, 1 \mathrm{H}), 2.58(\mathrm{~s}, 3 \mathrm{H}), 2.35(\mathrm{~s}, 3 \mathrm{H}) ;{ }^{13} \mathrm{C}$ NMR $(100 \mathrm{MHz}$, $\left.\mathrm{CDCl}_{3}\right) \delta 183.7,152.9,143.8,138.1,136.9,135.3,131.0,130.7,130.4,129.4,127.8,127.7,125.1$, 124.4, 120.5, 119.3, 110.0, 33.4, 21.6; IR (film): 3061, 2949, 2860, 1722, 1617, 1487,1345, 1204, 1099 , $1043 \mathrm{~cm}^{-1}$; HRMS (ESI): calcd for $\mathrm{C}_{23} \mathrm{H}_{20} \mathrm{~N}_{2} \mathrm{O}_{3} \mathrm{~S}-\mathrm{H}^{+}\left(\mathrm{M}-\mathrm{H}^{+}\right)$: 403.1122 , Found: 403.1119 .

(E)-N-((4-methoxyphenyl)(1-methyl-3-oxoindolin-2-ylidene)methyl)-4-methylbenzenesulfonamide (3j):

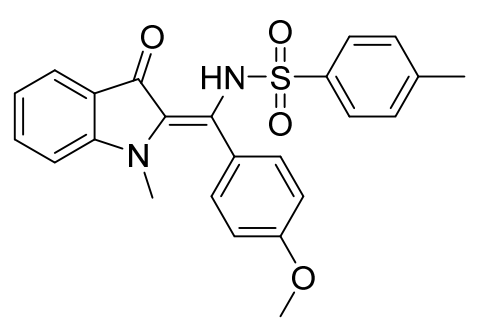

Red solid (24.3 mg, 28\%); M.p. 154-155 ${ }^{\circ} \mathrm{C} ;{ }^{1} \mathrm{H}$ NMR (400 MHz, $\left.\mathrm{CDCl}_{3}\right) \delta 12.09$ (s, $\left.1 \mathrm{H}\right), 7.75$ (d, J= $7.7 \mathrm{~Hz}, 1 \mathrm{H}), 7.48-7.38(\mathrm{~m}, 3 \mathrm{H}), 7.20(\mathrm{~d}, J=8.7 \mathrm{~Hz}, 2 \mathrm{H}), 7.13(\mathrm{~d}, J=8.1 \mathrm{~Hz}, 2 \mathrm{H}), 6.96-6.84(\mathrm{~m}, 4 \mathrm{H})$, 3.89 (s, 3H), $2.64(\mathrm{~s}, 3 \mathrm{H}), 2.35(\mathrm{~s}, 3 \mathrm{H}) ;{ }^{13} \mathrm{C}$ NMR $\left(100 \mathrm{MHz}, \mathrm{CDCl}_{3}\right) \delta 161.3,152.8,143.8,138.7$, 136.8, 135.0, 132.7, 129.3, 127.7, 125.2, 124.3, 122.8, 120.7, 119.3, 113.2, 110.1, 55.4, 33.6, 21.6; IR (film): 3059, 2961, 2830, 2113, 1737, 1603, 1545, 1427, 1210, 1085 $\mathrm{cm}^{-1}$; HRMS (ESI): calcd for $\mathrm{C}_{24} \mathrm{H}_{22} \mathrm{~N}_{2} \mathrm{O}_{4} \mathrm{~S}-\mathrm{H}^{+}\left(\mathrm{M}-\mathrm{H}^{+}\right)$: 433.1228, Found: 433.1223 .

(E)-N-((3,5-bis(trifluoromethyl)phenyl)(1-methyl-3-oxoindolin-2-ylidene)methyl)-4-methylbenzenesulfonamide (3k):<smiles>Cc1ccc(S(=O)(=O)NC(=C2C(=O)c3ccccc3N2C)c2cc(C(F)(F)F)cc(C(F)(F)F)c2)cc1</smiles>

Red solid (56.2 mg, 52\%); M.p. 189-190 ${ }^{\circ} \mathrm{C} ;{ }^{1} \mathrm{H}$ NMR (400 MHz, $\left.\mathrm{CDCl}_{3}\right) \delta 11.87$ (s, 1H), 7.95 (s, 1H), $7.77(\mathrm{~d}, J=7.7 \mathrm{~Hz}, 1 \mathrm{H}), 7.69$ (s, 2H), $7.52(\mathrm{t}, J=1.4 \mathrm{~Hz}, 1 \mathrm{H}), 7.38-7.33(\mathrm{~m}, 2 \mathrm{H}), 7.15(\mathrm{~d}, J=8.0 \mathrm{~Hz}$, 2H), $6.98(\mathrm{t}, J=7.4 \mathrm{~Hz}, 1 \mathrm{H}), 6.87(\mathrm{~d}, J=8.3 \mathrm{~Hz}, 1 \mathrm{H}), 2.57$ (s, 3H), $2.36(\mathrm{~s}, 3 \mathrm{H}) ;{ }^{13} \mathrm{C} \mathrm{NMR}(100 \mathrm{MHz}$, $\left.\mathrm{CDCl}_{3}\right) \delta 185.8,153.8,144.6,136.4,136.3,133.2,131.5-131.0(\mathrm{~m}), 130.3,129.7,127.5,126.4,124.9$, 124.2, $123.71-123.48$ (m), 121.5, 120.5, 120.1, 110.0, 34.1, 21.5; IR (film): 3044, 2967, 2917, 1929 , 1685, 1479, 1281, 1172, 1086, 864cm ${ }^{-1}$; HRMS (ESI): calcd for $\mathrm{C}_{25} \mathrm{H}_{18} \mathrm{~F}_{6} \mathrm{~N}_{2} \mathrm{O}_{3} \mathrm{~S}-\mathrm{H}^{+}\left(\mathrm{M}-\mathrm{H}^{+}\right): 539.0870$, Found: 539.0867.

(E)-N-((4-formylphenyl)(1-methyl-3-oxoindolin-2-ylidene)methyl)-4-methylbenzenesulfonamide (3i):<smiles>Cc1ccc(S(=O)(=O)N/C(=C2/C(=O)c3ccccc3N2C)c2ccc(C=O)cc2)cc1</smiles> 
Red solid (52.7 mg, 61\%); M.p. 170-171 ${ }^{0} \mathrm{C} ;{ }^{1} \mathrm{H}$ NMR (400 MHz, $\left.\mathrm{CDCl}_{3}\right) \delta 11.93$ (s, 1H), $10.10(\mathrm{~s}, 1 \mathrm{H})$, $7.91(\mathrm{~d}, J=8.2 \mathrm{~Hz}, 2 \mathrm{H}), 7.74(\mathrm{~d}, J=7.7 \mathrm{~Hz}, 1 \mathrm{H}), 7.51-7.45(\mathrm{~m}, 3 \mathrm{H}), 7.42(\mathrm{~d}, J=8.3 \mathrm{~Hz}, 2 \mathrm{H}), 7.16$ (d, $J=8.1 \mathrm{~Hz}, 2 \mathrm{H}), 6.95(\mathrm{t}, J=7.4 \mathrm{~Hz}, 1 \mathrm{H}), 6.86(\mathrm{~d}, J=8.3 \mathrm{~Hz}, 1 \mathrm{H}), 2.59$ (s, 3H), $2.36(\mathrm{~s}, 3 \mathrm{H}) ;{ }^{13} \mathrm{C} \mathrm{NMR}$ $\left(100 \mathrm{MHz}, \mathrm{CDCl}_{3}\right) \delta 191.5,185.0,153.5,144.2,137.1,136.6,135.9,134.0,131.8,129.5,128.8,127.6$, 126.0, 124.6, 120.5, 119.8, 110.1, 34.0, 21.6; IR (film): 3387, 2952, 2923, 2851, 2113, 1739, 1612, 1383, 1158, $1085 \mathrm{~cm}^{-1}$; HRMS (ESI): calcd for $\mathrm{C}_{24} \mathrm{H}_{20} \mathrm{~N}_{2} \mathrm{O}_{4} \mathrm{~S}-\mathrm{H}^{+}\left(\mathrm{M}-\mathrm{H}^{+}\right)$: 431.1071, Found: 431.1068 .

(E)-4-methyl-N-((1-methyl-3-oxoindolin-2-ylidene)(naphthalen-2-yl)methyl)benzenesulfonamide (3m):

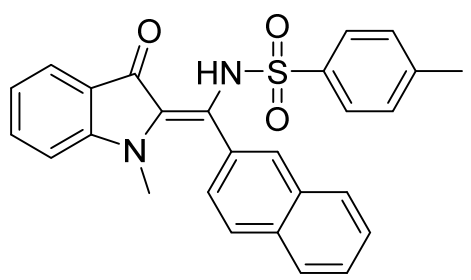

Red solid (43.6 mg, 48\%); M.p. $182-183{ }^{\circ} \mathrm{C}$; ${ }^{1} \mathrm{H}$ NMR (400 MHz, $\left.\mathrm{CDCl}_{3}\right) \delta 12.21$ (s, 1H), 7.94-7.82 (m, 2H), 7.81-7.69 (m, 2H), $7.63-7.52(\mathrm{~m}, 3 \mathrm{H}), 7.50-7.43(\mathrm{~m}, 2 \mathrm{H}), 7.35-7.30(\mathrm{~m}, 2 \mathrm{H}), 7.04(\mathrm{~d}, J=8.0$ $\mathrm{Hz}, 2 \mathrm{H}), 6.94(\mathrm{t}, J=7.5 \mathrm{~Hz}, 1 \mathrm{H}), 6.85(\mathrm{~d}, J=8.3 \mathrm{~Hz}, 1 \mathrm{H}), 2.55(\mathrm{~s}, 3 \mathrm{H}), 2.33(\mathrm{~s}, 3 \mathrm{H}),{ }^{13} \mathrm{C}$ NMR $(100$ $\left.\mathrm{MHz}, \mathrm{CDCl}_{3}\right) \delta 184.1,153.1,143.8,137.4,136.9,135.4,133.8,132.1,131.1,129.3,128.5,128.2$, 128.0, 127.8, 127.7, 127.7, 127.4, 126.8, 125.6, 124.5, 120.6, 119.4, 110.0, 33.7, 21.6;IR (film): 3056, 2936, 1908, 1650, 1617, 1485, 1341, 1184, 1085, $740 \mathrm{~cm}^{-1}$; HRMS (ESI): calcd for $\mathrm{C}_{27} \mathrm{H}_{22} \mathrm{~N}_{2} \mathrm{O}_{3} \mathrm{~S}-\mathrm{H}^{+}$ $\left(\mathrm{M}-\mathrm{H}^{+}\right)$: 453.1278, Found: 453.1280 .

(E)-4-methyl- $N$-((1-methyl-3-oxoindolin-2-ylidene)(pyren-1-yl)methyl)benzenesulfonamide (3n):<smiles>Cc1ccc(S(=O)(=O)N/C(=C2/C(=O)c3ccccc3N2C)c2ccc3ccc4cccc5ccc2c3c45)cc1</smiles>

Red solid (65.5 mg, 62\%); M.p. 139-140 ${ }^{\circ}$; ${ }^{1} \mathrm{H}$ NMR (400 MHz, $\left.\mathrm{CDCl}_{3}\right) \delta 12.82(\mathrm{~s}, 1 \mathrm{H}), 8.27$ (d, $J=$ $7.5 \mathrm{~Hz}, 1 \mathrm{H}), 8.22-8.10(\mathrm{~m}, 4 \mathrm{H}), 8.08-8.01(\mathrm{~m}, 2 \mathrm{H}), 7.86(\mathrm{~d}, J=7.7 \mathrm{~Hz}, 1 \mathrm{H}), 7.79(\mathrm{~d}, J=9.2 \mathrm{~Hz}$, $1 \mathrm{H}), 7.53(\mathrm{~d}, J=9.2 \mathrm{~Hz}, 1 \mathrm{H}), 7.47-7.39(\mathrm{~m}, 1 \mathrm{H}), 7.00(\mathrm{~d}, J=8.2 \mathrm{~Hz}, 2 \mathrm{H}), 6.94(\mathrm{t}, J=7.4 \mathrm{~Hz}, 1 \mathrm{H}), 6.73$ $(\mathrm{d}, J=8.3 \mathrm{~Hz}, 1 \mathrm{H}), 6.56(\mathrm{~d}, J=8.0 \mathrm{~Hz}, 2 \mathrm{H}), 2.16(\mathrm{~s}, 3 \mathrm{H}), 1.89(\mathrm{~s}, 3 \mathrm{H}) ;{ }^{13} \mathrm{C} \mathrm{NMR}\left(100 \mathrm{MHz}, \mathrm{CDCl}_{3}\right) \delta$ 183.6, 152.6, 143.4, 136.4, 136.3, 135.4, 132.7, 131.1, 130.5, 130.1, 129.4, 129.0, 128.8, 128.6, 127.4, 127.3, 126.4, 126.1, 125.9, 125.6, 124.6, 124.2, 124.1, 124.1, 123.9, 123.7, 120.4, 119.2, 109.7, 32.1, 21.0; IR(film): 3047, 2941, 1921, 1742, 1614, 1486, 1323, 1197, 1043, $848 \mathrm{~cm}^{-1}$; HRMS (ESI): calcd for $\mathrm{C}_{33} \mathrm{H}_{24} \mathrm{~N}_{2} \mathrm{O}_{3} \mathrm{~S}+\mathrm{H}^{+}\left([\mathrm{M}+\mathrm{H}]^{+}\right): 529.1580$, Found: 529.1577 . 
<smiles>Cc1ccc(S(=O)(=O)NC(=C2C(=O)c3ccccc3N2C)c2ccco2)cc1</smiles>

Red solid (56.8 mg, 72\%); M.p. 162-163 ${ }^{0} \mathrm{C} ;{ }^{1} \mathrm{H}$ NMR (400 MHz, $\left.\mathrm{CDCl}_{3}\right) \delta 11.42$ (s, $\left.1 \mathrm{H}\right), 7.70$ (d, J= $7.8 \mathrm{~Hz}, 1 \mathrm{H}), 7.59(\mathrm{~s}, 1 \mathrm{H}), 7.55-7.41(\mathrm{~m}, 3 \mathrm{H}), 7.17(\mathrm{~d}, J=7.9 \mathrm{~Hz}, 2 \mathrm{H}), 6.97-6.85(\mathrm{~m}, 2 \mathrm{H}), 6.82(\mathrm{~d}, J=$ $3.5 \mathrm{~Hz}, 1 \mathrm{H}), 6.61(\mathrm{~s}, 1 \mathrm{H}), 2.79(\mathrm{~s}, 3 \mathrm{H}), 2.35(\mathrm{~s}, 3 \mathrm{H}),{ }^{13} \mathrm{C} \mathrm{NMR}\left(150 \mathrm{MHz}, \mathrm{CDCl}_{3}\right) \delta 187.5,155.8,147.4$, 146.6, 145.5, 138.8, 138.4, 132.1, 130.2, 128.7, 127.1, 126.7, 123.1, 122.3, 120.6, 114.9, 112.6, 35.2, 24.3; IR (film): 3063, 2944, 1920, 1619, 1485, 1469, 1202, 1102, 1043, $885 \mathrm{~cm}^{-1}$; HRMS (ESI): calcd for $\mathrm{C}_{21} \mathrm{H}_{18} \mathrm{~N}_{2} \mathrm{O}_{4} \mathrm{~S}-\mathrm{H}^{+}\left(\mathrm{M}-\mathrm{H}^{+}\right)$: 393.0915, Found: 393.0915 .

(E)-4-methyl- $N$-((1-methyl-3-oxoindolin-2-ylidene)(thiophen-2-yl)methyl)benzenesulfonamide (3p):<smiles>Cc1ccc(S(=O)(=O)NC(=C2C(=O)c3ccccc3N2C)c2cccs2)cc1</smiles>

Red solid (45.1 mg, 55\%); M.p. 173-174 ${ }^{0} \mathrm{C} ;{ }^{1} \mathrm{H}$ NMR (400 MHz, $\left.\mathrm{CDCl}_{3}\right) \delta 11.77$ (s, 1H), 7.73 (d, $J=$ $7.7 \mathrm{~Hz}, 1 \mathrm{H}), 7.61-7.56(\mathrm{~m}, 2.1 \mathrm{~Hz}, 1 \mathrm{H}), 7.52-7.44(\mathrm{~m}, 3 \mathrm{H}), 7.16(\mathrm{~d}, J=8.1 \mathrm{~Hz}, 2 \mathrm{H}), 7.10(\mathrm{dd}, J=5.0$, $2.8 \mathrm{~Hz}, 2 \mathrm{H}), 6.97-6.86(\mathrm{~m}, 2 \mathrm{H}), 2.73(\mathrm{~s}, 3 \mathrm{H}), 2.35(\mathrm{~s}, 3 \mathrm{H}) ;{ }^{13} \mathrm{C} \mathrm{NMR}\left(150 \mathrm{MHz}, \mathrm{CDCl}_{3}\right) \delta 186.4$, 155.8, 146.5, 139.1, 138.1, 135.6, 134.1, 133.3, 132.0, 130.4, 129.4, 129.0, 127.1, 123.2, 122.3, 112.8, 35.6, 24.2; IR (film): 3067, 2925, 2253, 1777, 1616, 1485, 1336, 1185, 1085, $884 \mathrm{~cm}^{-1}$; HRMS (ESI): calcd for $\mathrm{C}_{21} \mathrm{H}_{18} \mathrm{~N}_{2} \mathrm{O}_{3} \mathrm{~S}_{2}-\mathrm{H}^{+}\left(\mathrm{M}-\mathrm{H}^{+}\right)$: 409.0686, Found: 409.0681 .

(E)-4-methyl-N-((1-methyl-3-oxoindolin-2-ylidene)(thiophen-3-yl)methyl)benzenesulfonamide (3q):<smiles>Cc1ccc(S(=O)(=O)NC(=C2C(=O)c3ccccc3N2C)c2ccsc2)cc1</smiles>

Red solid (35.3 mg, 43\%); M.p. 167-168 ${ }^{0} \mathrm{C} ;{ }^{1} \mathrm{H}$ NMR (400 MHz, $\left.\mathrm{CDCl}_{3}\right) \delta 12.16$ (s, $\left.1 \mathrm{H}\right), 7.74$ (d, J= $7.8 \mathrm{~Hz}, 1 \mathrm{H}), 7.50-7.43(\mathrm{~m}, 1 \mathrm{H}), 7.41(\mathrm{~d}, J=8.3 \mathrm{~Hz}, 2 \mathrm{H}), 7.35-7.31(\mathrm{~m}, 1 \mathrm{H}), 7.29-7.26(\mathrm{~m}, 1 \mathrm{H})$, $7.14(\mathrm{~d}, J=8.1 \mathrm{~Hz}, 2 \mathrm{H}), 7.08-7.04(\mathrm{~m}, 1 \mathrm{H}), 6.96-6.87(\mathrm{~m}, 2 \mathrm{H}), 2.67(\mathrm{~s}, 3 \mathrm{H}), 2.35(\mathrm{~s}, 3 \mathrm{H}),{ }^{13} \mathrm{C} \mathrm{NMR}$ $\left(100 \mathrm{MHz}, \mathrm{CDCl}_{3}\right) \delta 183.2,152.8,143.8,136.7,135.2,133.7,130.70$ (s), 130.0, 129.8, 129.4, 127.6, 125.4, 125.3, 124.3, 120.5, 119.4, 110.2, 33.1, 21.6; IR (film): 3106, 2924, 2253, 1914, 1644, 1486, 1184, 1099, $909 \mathrm{~cm}^{-1}$; HRMS (ESI): calcd for $\mathrm{C}_{21} \mathrm{H}_{18} \mathrm{~N}_{2} \mathrm{O}_{3} \mathrm{~S}_{2}-\mathrm{H}^{+}\left(\mathrm{M}-\mathrm{H}^{+}\right)$: 409.0686, Found: 409.0689. 
(E)-N-((5-fluoro-1-methyl-3-oxoindolin-2-ylidene)(2-fluorophenyl)methyl)-4-methylbenzenesulfonamide( 3r):<smiles>Cc1ccc(S(=O)(=O)N/C(=C2/C(=O)c3cc(F)ccc3N2C)c2ccccc2F)cc1</smiles>

Red solid (71.3 mg, 81\%); M.p. 180-181 ${ }^{0} \mathrm{C} ;{ }^{1} \mathrm{H}$ NMR (400 MHz, $\left.\mathrm{CDCl}_{3}\right) \delta 12.23(\mathrm{~s}, 1 \mathrm{H}), 7.54-7.46$ (m, 1H), $7.44(\mathrm{~d}, J=8.1 \mathrm{~Hz}, 2 \mathrm{H}), 7.41-7.36(\mathrm{~m}, 1 \mathrm{H}), 7.28-7.13(\mathrm{~m}, 5 \mathrm{H}), 7.07$ (t, $J=8.9 \mathrm{~Hz}, 1 \mathrm{H})$, 6.85-6.79 (m, 1H), $2.65(\mathrm{~s}, 3 \mathrm{H}), 2.37(\mathrm{~s}, 3 \mathrm{H}) ;{ }^{13} \mathrm{C} \mathrm{NMR}\left(100 \mathrm{MHz}, \mathrm{CDCl}_{3}\right) \delta 181.9,160.1(\mathrm{~d}, J=251.5$ $\mathrm{Hz}), 156.8$ (d, $J=239.8 \mathrm{~Hz}), 148.9,144.0,136.9,133.2,132.8(\mathrm{~d}, J=8.2 \mathrm{~Hz}), 132.6(\mathrm{~d}, J=2.0 \mathrm{~Hz})$, 129.5, 127.5, 125.7, 123.7 (d, $J=3.0 \mathrm{~Hz}), 123.5$ (d, $J=25.8 \mathrm{~Hz}), 120.1$ (d, $J=8.2 \mathrm{~Hz}), 118.7(\mathrm{~d}, J=$ $19.4 \mathrm{~Hz}), 115.6(\mathrm{~d}, J=21.0 \mathrm{~Hz}), 110.9$ (d, $J=8.0 \mathrm{~Hz}), 109.2$ (d, $J=23.2 \mathrm{~Hz}), 32.3,21.6$; IR (film): 3062, 2970, 2923, 2244, 1616, 1493, 1165, 1085, 881, $734 \mathrm{~cm}^{-1}$; HRMS (ESI): calcd for $\mathrm{C}_{23} \mathrm{H}_{18} \mathrm{~F}_{2} \mathrm{~N}_{2} \mathrm{O}_{3} \mathrm{~S}-\mathrm{H}^{+}\left(\mathrm{M}-\mathrm{H}^{+}\right)$: 439.0933, Found: 439.0939 .

(E)- $N$-((2-fluorophenyl)(5-methoxy-1-methyl-3-oxoindolin-2-ylidene)methyl)-4methylbenzenesulfonamide (3s):<smiles>COc1ccc2c(c1)C(=O)C(=C(NS(=O)(=O)c1ccc(C)cc1)c1ccccc1F)N2C</smiles>

Red solid ( 59.7 mg, 66\%); M.p. 156-157 ${ }^{0} \mathrm{C}$; ${ }^{1} \mathrm{H}$ NMR (400 MHz, $\mathrm{CDCl}_{3}$ ) $\delta 12.30$ (s, 1H), $7.52-7.42$ $(\mathrm{m}, 3 \mathrm{H}), 7.29-7.03(\mathrm{~m}, 7 \mathrm{H}), 6.80(\mathrm{~d}, J=8.9 \mathrm{~Hz}, 1 \mathrm{H}), 3.80(\mathrm{~s}, 3 \mathrm{H}), 2.62(\mathrm{~s}, 3 \mathrm{H}), 2.36(\mathrm{~s}, 3 \mathrm{H}) ;{ }^{13} \mathrm{C}$ NMR $\left(100 \mathrm{MHz}, \mathrm{CDCl}_{3}\right) \delta 182.6,160.2(\mathrm{~d}, J=251.6 \mathrm{~Hz}), 153.6,148.5,143.9,136.9,132.7,132.6$, 132.6, 132.1, 129.5, 127.5, 126.2 (d, $J=7.8 \mathrm{~Hz}), 123.6$ (d, $J=3.5 \mathrm{~Hz}), 120.0,118.9$ (d, $J=15.2 \mathrm{~Hz})$, 115.5 (d, $J=21.1 \mathrm{~Hz}), 111.2,104.4,55.9,32.4,21.6$; IR (film): 3062, 2945, 2839, 2247, 1644, 1615 , 1495, 1337, 1281, $1165 \mathrm{~cm}^{-1}$; HRMS (ESI): calcd for $\mathrm{C}_{24} \mathrm{H}_{21} \mathrm{FN}_{2} \mathrm{O}_{4} \mathrm{~S}-\mathrm{H}^{+}\left(\mathrm{M}-\mathrm{H}^{+}\right)$: 451.1133, Found: 451.1130 .

(E)-N-((2-fluorophenyl)(1-methyl-3-oxoindolin-2-ylidene)methyl)methanesulfonamide (3t):<smiles>CN1C(=C(NS(C)(=O)=O)c2ccccc2F)C(=O)c2ccccc21</smiles>

Red solid ( $38.1 \mathrm{mg}, 55 \%$ ); M.p. 177-178 ${ }^{0} \mathrm{C} ;{ }^{1} \mathrm{H}$ NMR (400 MHz, $\left.\mathrm{CDCl}_{3}\right) \delta 11.69$ (s, 1H), 7.77 (d, $J=$ $7.7 \mathrm{~Hz}, 1 \mathrm{H}), 7.66-7.43(\mathrm{~m}, 3 \mathrm{H}), 7.37-7.16(\mathrm{~m}, 2 \mathrm{H}), 7.00-6.89(\mathrm{~m}, 2 \mathrm{H}), 2.97(\mathrm{~s}, 3 \mathrm{H}), 2.77(\mathrm{~s}, 3 \mathrm{H})$; ${ }^{13} \mathrm{C}$ NMR $\left(100 \mathrm{MHz}, \mathrm{CDCl}_{3}\right) \delta 184.2,160.1(\mathrm{~d}, J=249.3 \mathrm{~Hz}), 153.1,136.0,132.7(\mathrm{~d}, J=8.3 \mathrm{~Hz}), 132.6$ $(\mathrm{d}, J=1.9 \mathrm{~Hz}), 129.4,126.2,124.6,124.2(\mathrm{~d}, J=3.5 \mathrm{~Hz}), 120.1,119.8(\mathrm{~d}, J=15.2 \mathrm{~Hz}), 119.6,115.8$ (d, $J=21.1 \mathrm{~Hz}$ ), 109.8, 41.9, 32.0; IR (film): 3064, 3006, 2933, 2848, 1735, 1620, 1487, 1344, 1201, $1044 \mathrm{~cm}^{-1}$; HRMS (ESI): calcd for $\mathrm{C}_{17} \mathrm{H}_{15} \mathrm{FN}_{2} \mathrm{O}_{3} \mathrm{~S}-\mathrm{H}^{+}\left(\mathrm{M}-\mathrm{H}^{+}\right)$: 345.0715, Found: 345.0728 . 


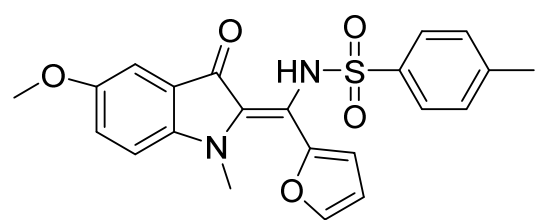

Purple solid (43.3mg, 51\%); M.p. 197-198 ${ }^{0} \mathrm{C}$; ${ }^{1} \mathrm{H}$ NMR (600 MHz, $\left.\mathrm{CDCl}_{3}\right) \delta 11.41$ (s, 1H), 7.60 (s, $1 \mathrm{H}), 7.52(\mathrm{~d}, J=8.1 \mathrm{~Hz}, 2 \mathrm{H}), 7.18(\mathrm{~d}, J=8.0 \mathrm{~Hz}, 2 \mathrm{H}), 7.15-7.10(\mathrm{~m}, 2 \mathrm{H}), 6.92-6.81(\mathrm{~m}, 2 \mathrm{H}), 6.64-$ $6.60(\mathrm{~m}, 1 \mathrm{H}), 3.80(\mathrm{~s}, 3 \mathrm{H}), 2.77(\mathrm{~s}, 3 \mathrm{H}), 2.35(\mathrm{~s}, 3 \mathrm{H}) ;{ }^{13} \mathrm{C} \mathrm{NMR}\left(150 \mathrm{MHz}, \mathrm{CDCl}_{3}\right) \delta 183.2,153.8$, 148.8, 144.9, 143.9, 143.0, 136.2, 129.4, 127.5, 126.7, 125.9, 120.4, 118.3, 112.3, 111.5, 104.4, 55.8, 33.1, 21.6; IR (film): 3405, 2924, 1734, 1646, 1605, 1494, 1336, 1280, 1185, $884 \mathrm{~cm}^{-1}$; HRMS (ESI): calcd for $\mathrm{C}_{22} \mathrm{H}_{20} \mathrm{~N}_{2} \mathrm{O}_{5} \mathrm{~S}-\mathrm{H}^{+}\left(\mathrm{M}-\mathrm{H}^{+}\right)$: 423.1020 , Found: 423.1017 .

(E)- $N$-((5-bromo-1-methyl-3-oxoindolin-2-ylidene)(furan-2-yl)methyl)-4-methylbenzenesulfonamide (3v):<smiles>Cc1ccc(S(=O)(=O)N/C(=C2/C(=O)c3cc(Br)ccc3N2C)c2ccco2)cc1</smiles>

Red solid (69.9 mg, 74\%); M.p. 179-180 ${ }^{0} \mathrm{C} ;{ }^{1} \mathrm{H}$ NMR (400 MHz, $\left.\mathrm{CDCl}_{3}\right) \delta 11.29$ (s, 1H), 7.77 (d, $J=$ $1.9 \mathrm{~Hz}, 1 \mathrm{H}), 7.60(\mathrm{~d}, J=1.1 \mathrm{~Hz}, 1 \mathrm{H}), 7.54-7.46(\mathrm{~m}, 3 \mathrm{H}), 7.18(\mathrm{~d}, J=8.1 \mathrm{~Hz}, 2 \mathrm{H}), 6.88(\mathrm{~d}, J=3.1 \mathrm{~Hz}$, $1 \mathrm{H}), 6.79(\mathrm{~d}, J=8.7 \mathrm{~Hz}, 1 \mathrm{H}), 6.65-6.61(\mathrm{~m}, 1 \mathrm{H}), 2.79(\mathrm{~s}, 3 \mathrm{H}), 2.35(\mathrm{~s}, 3 \mathrm{H}) ;{ }^{13} \mathrm{C}$ NMR $(100 \mathrm{MHz}$, $\left.\mathrm{CDCl}_{3}\right) \delta 151.0,145.2,144.1,142.6,137.7,136.2,129.5,127.6,126.7,125.4,121.8,118.7,112.5$, 112.2, 111.7, 32.6, 21.6; IR (film): 3128, 3036, 2920, 2836, 1867, 1747, 1595, 1471, 1302, $1026 \mathrm{~cm}^{-1}$; HRMS (ESI): calcd for $\mathrm{C}_{21} \mathrm{H}_{17} \mathrm{BrN}_{2} \mathrm{O}_{4} \mathrm{~S}-\mathrm{H}^{+}\left(\mathrm{M}-\mathrm{H}^{+}\right)$: 471.0020 , Found: 471.0017.

(E)-N-((5-fluoro-1-methyl-3-oxoindolin-2-ylidene)(furan-2-yl)methyl)-4-methylbenzenesulfonamide (3w):<smiles>Cc1ccc(S(=O)(=O)N/C(=C2/C(=O)c3cc(F)ccc3N2C)c2ccco2)cc1</smiles>

Red solid (68.4 mg, 83\%); M.p. 164-165 ${ }^{0} \mathrm{C} ;{ }^{1} \mathrm{H}$ NMR (400 MHz, $\left.\mathrm{CDCl}_{3}\right) \delta 11.28$ (s, 1H), 7.60 (d, $J=$ $1.4 \mathrm{~Hz}, 1 \mathrm{H}), 7.51(\mathrm{~d}, J=8.3 \mathrm{~Hz}, 2 \mathrm{H}), 7.35-7.30(\mathrm{~m}, 1 \mathrm{H}), 7.25-7.15(\mathrm{~m}, 3 \mathrm{H}), 6.92-6.84(\mathrm{~m}, 2 \mathrm{H})$, 6.65-6.60 (m, 1H), $2.80(\mathrm{~s}, 3 \mathrm{H}), 2.35$ (s, 3H); ${ }^{13} \mathrm{C} \mathrm{NMR}\left(100 \mathrm{MHz}, \mathrm{CDCl}_{3}\right) \delta 157.0(\mathrm{~d}, J=240.1 \mathrm{~Hz})$, 149.0, 145.2, 144.1, 142.8, 136.3, 129.5, 127.5, 126.3, 126.1, 123.3 (d, $J=25.7 \mathrm{~Hz}), 120.4$ (d, $J=8.1$ Hz), 118.8 (s), 112.5 (s), 111.4 (d, $J=7.9 \mathrm{~Hz}), 109.1$ (d, $J=23.3 \mathrm{~Hz}), 32.9$, 21.6; IR (film): 3131, $3062,2923,2119,1747,1650,1492,1340,1165,1039 \mathrm{~cm}^{-1}$; HRMS (ESI): calcd for $\mathrm{C}_{21} \mathrm{H}_{17} \mathrm{FN}_{2} \mathrm{O}_{4} \mathrm{~S}-\mathrm{H}^{+}$ $\left(\mathrm{M}-\mathrm{H}^{+}\right)$: 411.0820, Found: 411.0813 . 
<smiles>CCN1/C(=C(\NS(=O)(=O)c2ccc(C)cc2)c2ccco2)C(=O)c2ccccc21</smiles>

Red solid (58.8 mg, 72\%); M.p. $148-149{ }^{0} \mathrm{C} ;{ }^{1} \mathrm{H}$ NMR $\left(400 \mathrm{MHz}, \mathrm{CDCl}_{3}\right) \delta 11.68$ (s, $\left.1 \mathrm{H}\right), 7.70$ (d, J= $7.7 \mathrm{~Hz}, 1 \mathrm{H}), 7.58(\mathrm{~s}, 1 \mathrm{H}), 7.52(\mathrm{~d}, J=8.0 \mathrm{~Hz}, 2 \mathrm{H}), 7.46(\mathrm{~d}, J=1.0 \mathrm{~Hz}, 1 \mathrm{H}), 7.18(\mathrm{~d}, J=8.1 \mathrm{~Hz}, 2 \mathrm{H})$, $6.98-6.88(\mathrm{~m}, 2 \mathrm{H}), 6.81(\mathrm{~d}, J=3.4 \mathrm{~Hz}, 1 \mathrm{H}), 6.62-6.58(\mathrm{~m}, 1 \mathrm{H}), 3.17$ (q, $J=7.0 \mathrm{~Hz}, 2 \mathrm{H}), 2.35(\mathrm{~s}, 3 \mathrm{H})$, $0.83(\mathrm{t}, J=7.0 \mathrm{~Hz}, 3 \mathrm{H}) ;{ }^{13} \mathrm{C} \mathrm{NMR}\left(100 \mathrm{MHz}, \mathrm{CDCl}_{3}\right) \delta 185.3,152.5,144.7,143.9,142.8,136.3,135.7$, 129.5, 127.6, 124.9, 124.6, 124.5, 121.3, 119.8, 117.6, 112.2, 110.7, 39.4, 21.6, 12.4; IR (film): 2959, 2925, 2866, 2112, 1736, 1611, 1482, 1346, 1162, $1045 \mathrm{~cm}^{-1}$; HRMS (ESI):calcd for $\mathrm{C}_{22} \mathrm{H}_{20} \mathrm{~N}_{2} \mathrm{O}_{4} \mathrm{~S}-\mathrm{H}^{+}$ $\left(\mathrm{M}-\mathrm{H}^{+}\right)$: 407.1071, Found: 407.1061.

(E)-N-(furan-2-yl(3-oxo-1-phenylindolin-2-ylidene)methyl)-4-methylbenzenesulfonamide (3y):<smiles>Cc1ccc(S(=O)(=O)N/C(=C2/C(=O)c3ccccc3N2c2ccccc2)c2ccco2)cc1</smiles>

Red solid (42.0 mg, 46\%); M.p. 136-137 ${ }^{0} \mathrm{C} ;{ }^{1} \mathrm{H}$ NMR (400 MHz, $\left.\mathrm{CDCl}_{3}\right) \delta 11.67$ (s, 1H), 7.79 (d, $J=$ $7.7 \mathrm{~Hz}, 1 \mathrm{H}), 7.49(\mathrm{~d}, J=8.3 \mathrm{~Hz}, 2 \mathrm{H}), 7.42-7.34(\mathrm{~m}, 1 \mathrm{H}), 7.17(\mathrm{t}, J=7.2 \mathrm{~Hz}, 4 \mathrm{H}), 7.13-7.07(\mathrm{~m}, 1 \mathrm{H})$, $7.03-6.90(\mathrm{~m}, 5 \mathrm{H}), 6.52(\mathrm{~d}, J=3.5 \mathrm{~Hz}, 1 \mathrm{H}), 6.13-6.08(\mathrm{~m}, 1 \mathrm{H}), 2.35(\mathrm{~s}, 3 \mathrm{H}) ;{ }^{13} \mathrm{C}$ NMR $(100 \mathrm{MHz}$, $\left.\mathrm{CDCl}_{3}\right) \delta 184.5,152.1,144.9,143.9,1412.0,138.1,136.2,135.4,129.4,128.9,127.6,126.6,126.5$, 126.0, 124.4, 123.9, 120.9, 120.7, 118.6, 111.5, 110.9, 21.6; IR (film): 3135, 3036, 2917, 2102, 1899, 1614, 1497, 1365, 1198, $1183 \mathrm{~cm}^{-1}$; HRMS (ESI): calcd for $\mathrm{C}_{26} \mathrm{H}_{20} \mathrm{~N}_{2} \mathrm{O}_{4} \mathrm{~S}-\mathrm{H}^{+}\left(\mathrm{M}-\mathrm{H}^{+}\right)$: 455.1071 , Found: 455.1070.

(E)-N-((1-benzyl-3-oxoindolin-2-ylidene)(furan-2-yl)methyl)-4-methylbenzenesulfonamide (3z):<smiles>Cc1ccc(S(=O)(=O)N/C(=C2/C(=O)c3ccccc3N2Cc2ccccc2)c2ccco2)cc1</smiles>

Red solid (69.6 mg, 74\%); M.p. 158-159 ${ }^{0} \mathrm{C} ;{ }^{1} \mathrm{H}$ NMR (400 MHz, $\left.\mathrm{CDCl}_{3}\right) \delta 11.59$ (s, 1H), 7.74 (d, $J=$ $7.7 \mathrm{~Hz}, 1 \mathrm{H}), 7.50(\mathrm{~d}, J=8.3 \mathrm{~Hz}, 2 \mathrm{H}), 7.44-7.37(\mathrm{~m}, 1 \mathrm{H}), 7.32(\mathrm{~d}, J=1.1 \mathrm{~Hz}, 1 \mathrm{H}), 7.22-7.15(\mathrm{~m}$, $5 \mathrm{H}), 6.95(\mathrm{t}, J=7.4 \mathrm{~Hz}, 1 \mathrm{H}), 6.85-6.75(\mathrm{~m}, 3 \mathrm{H}), 6.65(\mathrm{~d}, J=3.4 \mathrm{~Hz}, 1 \mathrm{H}), 6.48-6.43(\mathrm{~m}, 1 \mathrm{H}), 4.37(\mathrm{~s}$, 2H), $2.36(\mathrm{~s}, 3 \mathrm{H}) ;{ }^{13} \mathrm{C} \mathrm{NMR}\left(100 \mathrm{MHz}, \mathrm{CDCl}_{3}\right) \delta 184.0,152.0,143.8,142.9,141.5,135.4,135.2$, 
134.7, 128.4, 127.4, 126.6, 126.3, 125.0, 124.1, 124.0, 123.5, 120.0, 119.1, 117.0, 111.1, 110.1, 47.7, 20.6; IR (film): 3030, 2956, 2924, 2855, 2114, 1737, 1614, 1482, 1349, $1104 \mathrm{~cm}^{-1}$; HRMS (ESI): calcd for $\mathrm{C}_{27} \mathrm{H}_{22} \mathrm{~N}_{2} \mathrm{O}_{4} \mathrm{~S}-\mathrm{H}^{+}\left(\mathrm{M}-\mathrm{H}^{+}\right)$: 469.1228, Found: 469.1237 .

(E)-4-fluoro-N-(furan-2-yl(1-methyl-3-oxoindolin-2-ylidene)methyl)benzenesulfonamide (3A):<smiles>CN1/C(=C(/NS(=O)(=O)c2ccc(F)cc2)c2ccco2)C(=O)c2ccccc21</smiles>

Purple solid (62.1 mg, 78\%); M.p. 168-169 ${ }^{0} \mathrm{C} ;{ }^{1} \mathrm{H}$ NMR (400 MHz, $\left.\mathrm{CDCl}_{3}\right) \delta 11.36(\mathrm{~s}, 1 \mathrm{H}), 7.70-$ $7.62(\mathrm{~m}, 3 \mathrm{H}), 7.59$ (d, $J=1.1 \mathrm{~Hz}, 1 \mathrm{H}), 7.51-7.46(\mathrm{~m}, 1 \mathrm{H}), 7.09-7.03(\mathrm{~m}, 2 \mathrm{H}), 6.93-6.88(\mathrm{~m}, 2 \mathrm{H})$, 6.85-6.80 (m, 1H), 6.64-6.59(m, 1H), $2.81(\mathrm{~s}, 3 \mathrm{H}) ;{ }^{13} \mathrm{C} \mathrm{NMR}\left(100 \mathrm{MHz}, \mathrm{CDCl}_{3}\right) \delta 184.9,165.2(\mathrm{~d}, J=$ 255.4 Hz), 153.3, 144.9, 142.9, 136.0, 135.1, 130.5 (d, $J=9.5 \mathrm{~Hz}), 126.3$, 124.5, 123.4, 120.3, 119.8, 118.0, 116.0 (d, $J=22.7 \mathrm{~Hz}$ ), 112.4, 110.0, 32.5; IR (film): 3105, 3070, 2942, 2857, 1896, 1651, 1485, 1345, 1203, $1101 \mathrm{~cm}^{-1}$; HRMS (ESI): calcd for $\mathrm{C}_{20} \mathrm{H}_{15} \mathrm{FN}_{2} \mathrm{O}_{4} \mathrm{~S}-\mathrm{H}^{+}\left(\mathrm{M}-\mathrm{H}^{+}\right): 397.0664$, Found: 397.0660 .

(E)-4-chloro-N-(furan-2-yl(1-methyl-3-oxoindolin-2-ylidene)methyl)benzenesulfonamide (3B):<smiles>CN1/C(=C(/NS(=O)(=O)c2ccc(Cl)cc2)c2ccco2)C(=O)c2ccccc21</smiles>

Red solid (62.9 mg, 76\%); M.p. 130-131 ${ }^{0} \mathrm{C} ;{ }^{1} \mathrm{H}$ NMR (400 MHz, $\left.\mathrm{CDCl}_{3}\right) \delta 11.39(\mathrm{~s}, 1 \mathrm{H}), 7.69(\mathrm{~d}, J=$ $7.7 \mathrm{~Hz}, 1 \mathrm{H}), 7.61-7.55(\mathrm{~m}, 3 \mathrm{H}), 7.49$ (t, $J=7.4 \mathrm{~Hz}, 1 \mathrm{H}), 7.36(\mathrm{~d}, J=8.3 \mathrm{~Hz}, 2 \mathrm{H}), 6.98-6.87(\mathrm{~m}, 2 \mathrm{H})$, $6.82(\mathrm{~d}, J=3.4 \mathrm{~Hz}, 1 \mathrm{H}), 6.65-6.59(\mathrm{~m}, 1 \mathrm{H}), 2.81(\mathrm{~s}, 3 \mathrm{H}) ;{ }^{13} \mathrm{C} \mathrm{NMR}\left(100 \mathrm{MHz}, \mathrm{CDCl}_{3}\right) \delta 184.8,153.3$, 144.9, 142.8, 139.6, 137.6, 136.0, 129.1, 129.1, 126.3, 124.6, 123.4, 120.3, 119.8, 118.0, 112.4, 110.0, 32.5; IR (film): 3121, 3092, 1922, 1867, 1698, 1584, 1484, 1294, 1088, $827 \mathrm{~cm}^{-1}$; HRMS (MALDI-TOF): calcd for $\mathrm{C}_{20} \mathrm{H}_{15} \mathrm{ClN}_{2} \mathrm{O}_{4} \mathrm{~S}+\mathrm{H}^{+}\left([\mathrm{M}+\mathrm{H}]^{+}\right)$: 415.0514 , Found: 415.0540 .

(E)-N-(furan-2-yl(1-methyl-3-oxoindolin-2-ylidene)methyl)benzenesulfonamide (3C):<smiles>CN1/C(=C(/NS(=O)(=O)c2ccccc2)c2ccco2)C(=O)c2ccccc21</smiles>

Red solid (54.0 mg, 71\%); M.p. 157-158 ${ }^{0} \mathrm{C} ;{ }^{1} \mathrm{H}$ NMR (400 MHz, $\left.\mathrm{CDCl}_{3}\right) \delta 11.45(\mathrm{~s}, 1 \mathrm{H}), 7.71-7.62(\mathrm{~m}$, $3 \mathrm{H}), 7.59-7.55(\mathrm{~m}, 1 \mathrm{H}), 7.48$ (q, $J=7.3 \mathrm{~Hz}, 2 \mathrm{H}), 7.39$ (t, $J=7.7 \mathrm{~Hz}, 2 \mathrm{H}), 6.96-6.86(\mathrm{~m}, 2 \mathrm{H}), 6.80$ $(\mathrm{d}, J=3.4 \mathrm{~Hz}, 1 \mathrm{H}), 6.63-6.58(\mathrm{~m}, 1 \mathrm{H}), 2.79(\mathrm{~s}, 3 \mathrm{H}) ;{ }^{13} \mathrm{C} \mathrm{NMR}\left(100 \mathrm{MHz}, \mathrm{CDCl}_{3}\right) \delta 184.7,153.2$, $144.8,142.9$, 139.1, 135.8, 133.1, 128.8, 127.6, 126.1, 124.5, 124.0, 120.4, 119.7, 118.0, 112.3, 110.0, 
32.5; IR (film): 3143, 3064, 2940, 2842, 2110, 1619, 1484, 1343, 1202, $1086 \mathrm{~cm}^{-1}$; HRMS (ESI): calcd for $\mathrm{C}_{20} \mathrm{H}_{16} \mathrm{~N}_{2} \mathrm{O}_{4} \mathrm{~S}-\mathrm{H}^{+}\left(\mathrm{M}-\mathrm{H}^{+}\right)$: 379.0758 , Found: 379.0747 .

(E)-N-(furan-2-yl(1-methyl-3-oxoindolin-2-ylidene)methyl)methanesulfonamide (3D):<smiles>CN1/C(=C(\NS(C)(=O)=O)c2ccco2)C(=O)c2ccccc21</smiles>

Red solid (43.3 mg, 68\%); M.p. 158-159 ${ }^{0} \mathrm{C} ;{ }^{1} \mathrm{H}$ NMR (400 MHz, $\left.\mathrm{CDCl}_{3}\right) \delta 10.82(\mathrm{~s}, 1 \mathrm{H}), 7.76-7.72(\mathrm{~m}$, 1H), $7.71-7.68(\mathrm{~m}, 1 \mathrm{H}), 7.56-7.50(\mathrm{~m}, 1 \mathrm{H}), 7.00-6.95(\mathrm{~m}, 2 \mathrm{H}), 6.91(\mathrm{~d}, J=3.5 \mathrm{~Hz}, 1 \mathrm{H}), 6.92-6.87$ (m, 1H), $3.05(\mathrm{~s}, 3 \mathrm{H}), 2.95(\mathrm{~s}, 3 \mathrm{H}) ;{ }^{13} \mathrm{C} \mathrm{NMR}\left(100 \mathrm{MHz}, \mathrm{CDCl}_{3}\right) \delta 153.4,145.1,143.9,135.9,126.3$, 124.5, 123.9,120.6, 120.0, 117.7, 112.5, 110.2, 41.5, 33.1; IR (film): 3139, 3122, 3003, 2930, 1868, 1649, 1487, 1298, 1187, $1042 \mathrm{~cm}^{-1}$; HRMS (ESI): calcd for $\mathrm{C}_{15} \mathrm{H}_{14} \mathrm{~N}_{2} \mathrm{O}_{4} \mathrm{~S}-\mathrm{H}^{+}\left(\mathrm{M}-\mathrm{H}^{+}\right)$: 317.0602 , Found: 317.0594 .

$N$-(2,5-dimethyl-2,3-dihydropyrano[3,2-b]indol-4(5H)-ylidene)-4methylbenzenesulfonamide(5a):<smiles>CC1CC(=N[As])c2c(c3ccccc3n2C)O1</smiles>

Yellow solid (25.0 mg, 34\%); M.p. 190-191 ${ }^{0} \mathrm{C} ;{ }^{1} \mathrm{H}$ NMR (400 MHz, $\left.\mathrm{CDCl}_{3}\right) \delta 7.90(\mathrm{~d}, J=8.3 \mathrm{~Hz}, 2 \mathrm{H})$, $7.64(\mathrm{~d}, J=8.1 \mathrm{~Hz}, 1 \mathrm{H}), 7.42-7.36(\mathrm{~m}, 1 \mathrm{H}), 7.32(\mathrm{~d}, J=8.0 \mathrm{~Hz}, 2 \mathrm{H}), 7.19$ (d, $J=8.6 \mathrm{~Hz}, 1 \mathrm{H}), 7.04(\mathrm{t}, J$ $=7.5 \mathrm{~Hz}, 1 \mathrm{H}), 4.68-4.55(\mathrm{~m}, 1 \mathrm{H}), 3.83(\mathrm{~s}, 3 \mathrm{H}), 3.76(\mathrm{dd}, J=17.9,3.2 \mathrm{~Hz}, 1 \mathrm{H}), 3.10-3.00(\mathrm{~m}, 1 \mathrm{H}), 2.44$ (s, 3H), $1.60(\mathrm{~d}, J=6.3 \mathrm{~Hz}, 3 \mathrm{H}) ;{ }^{13} \mathrm{C}$ NMR $\left(100 \mathrm{MHz}, \mathrm{CDCl}_{3}\right) \delta 164.6,152.7,143.0,141.0,139.6$, 129.4, 129.3, 126.7, 120.5, 119.7, 117.2, 115.3, 110.0, 76.1, 37.7, 32.1, 21.6, 20.4; IR (film): 3041, 2976, 2899, 1614, 1556, 1404, 1379, 1297, 1085, $931 \mathrm{~cm}^{-1}$; HRMS (EI): calcd for $\mathrm{C}_{20} \mathrm{H}_{20} \mathrm{~N}_{2} \mathrm{O}_{3} \mathrm{~S}$ ([M] ${ }^{+}$): 368.1195, Found: 368.1195 .

$N$-(8-fluoro-2,5-dimethyl-2,3-dihydropyrano[3,2-b]indol-4(5H)-ylidene)-4-methylbenzenesulfonamide (5b):<smiles>CC1CC(=N[As])c2c(c3cc(F)ccc3n2C)O1</smiles>

Yellow solid (18.5 mg, 24\%); M.p. 200-201 ${ }^{0} \mathrm{C} ;{ }^{1} \mathrm{H}$ NMR (400 MHz, $\left.\mathrm{CDCl}_{3}\right) \delta 7.89$ (d, J=8.2 Hz, 2H), $7.33(\mathrm{~d}, J=8.2 \mathrm{~Hz}, 2 \mathrm{H}), 7.29-7.24(\mathrm{~m}, 1 \mathrm{H}), 7.19-7.11(\mathrm{~m}, 2 \mathrm{H}), 4.65-4.55(\mathrm{~m}, 1 \mathrm{H}), 3.81(\mathrm{~s}, 3 \mathrm{H}), 3.76$ (dd, $J=17.9,3.1 \mathrm{~Hz}, 1 \mathrm{H}), 3.11-2.99(\mathrm{~m}, 1 \mathrm{H}), 2.44(\mathrm{~s}, 3 \mathrm{H}), 1.60(\mathrm{~d}, J=6.3 \mathrm{~Hz}, 3 \mathrm{H}) ;{ }^{13} \mathrm{C}$ NMR $(100$ $\left.\mathrm{MHz}, \mathrm{CDCl}_{3}\right) \delta 164.8,157.1(\mathrm{~d}, J=238.4 \mathrm{~Hz}), 151.9(\mathrm{~d}, J=5.9 \mathrm{~Hz}), 143.2,139.4,137.5,129.5,126.7$, 118.4(d, $J=27.0 \mathrm{~Hz}), 118.4,114.9$ (d, $J=10.0 \mathrm{~Hz}), 111.2(\mathrm{~d}, J=9.0 \mathrm{~Hz}), 104.6(\mathrm{~d}, J=24.1 \mathrm{~Hz}), 76.1$, 
37.7, 32.3, 21.6, 20.4; IR (film): 3012, 2921, 2892, 1741, 1688, 1537, 1296, 1183, 1150, $1084 \mathrm{~cm}^{-1}$; HRMS (EI): calcd for $\mathrm{C}_{20} \mathrm{H}_{19} \mathrm{FN}_{2} \mathrm{O}_{3} \mathrm{~S}\left([\mathrm{M}]^{+}\right)$: 386.1100 , Found: 386.1100 .

$N$-(8-methoxy-2,5-dimethyl-2,3-dihydropyrano[3,2-b]indol-4(5H)-ylidene)-4-methylbenzenesulfonamide (5c):<smiles>COc1ccc2c(c1)c1c(n2C)C(=N[As])CC(C)O1</smiles>

Yellow solid (28.7 mg, 36\%); M.p. 197-198 C; ${ }^{1} \mathrm{H}$ NMR (400 MHz, $\left.\mathrm{CDCl}_{3}\right) \delta 7.90$ (d, J=8.3 Hz, 2H), $7.32(\mathrm{~d}, J=8.0 \mathrm{~Hz}, 2 \mathrm{H}), 7.12-7.04(\mathrm{~m}, 2 \mathrm{H}), 6.96(\mathrm{~d}, J=1.9 \mathrm{~Hz}, 1 \mathrm{H}), 4.67-4.54(\mathrm{~m}, 1 \mathrm{H}), 3.82(\mathrm{~s}, 3 \mathrm{H})$, 3.79 (s, 3H), $3.75(\mathrm{dd}, J=17.9,3.1 \mathrm{~Hz}, 1 \mathrm{H}), 3.12-2.98(\mathrm{~m}, 1 \mathrm{H}), 2.44(\mathrm{~s}, 3 \mathrm{H}), 1.60(\mathrm{~d}, J=6.3 \mathrm{~Hz}, 3 \mathrm{H})$; ${ }^{13} \mathrm{C}$ NMR $\left(100 \mathrm{MHz}, \mathrm{CDCl}_{3}\right) \delta 164.5,153.8,151.9,143.0,139.6,137.0,129.4,126.6,121.8,117.8$, 114.9, 111.2, 99.2, 76.0, 55.7, 37.8, 32.2, 21.6, 20.4; IR (film): 3027, 2929, 2941, 2893, 1893, 1530, 1301, 1180, 1086, $893 \mathrm{~cm}^{-1}$; HRMS (EI): calcd for $\mathrm{C}_{21} \mathrm{H}_{22} \mathrm{~N}_{2} \mathrm{O}_{4} \mathrm{~S}$ ([M] $]^{+}$): 398.1300, Found: 398.1306.

4-methyl-N-(5-methyl-2-phenyl-2,3-dihydropyrano[3,2-b]indol-4(5H)-ylidene)benzenesulfonamide (5d):<smiles>Cn1c2c(c3ccccc31)OC(c1ccccc1)C/C2=N/[As]</smiles>

Yellow solid (19.8 mg, 23\%); M.p. $195-196{ }^{0} \mathrm{C} ;{ }^{1} \mathrm{H}$ NMR (400 MHz, $\left.\mathrm{CDCl}_{3}\right) \delta 7.90$ (d, J= 8.2 Hz, 2H), $7.66(\mathrm{~d}, J=8.1 \mathrm{~Hz}, 1 \mathrm{H}), 7.54$ (d, $J=6.8 \mathrm{~Hz}, 2 \mathrm{H}), 7.48-7.37(\mathrm{~m}, 4 \mathrm{H}), 7.33$ (d, $J=8.1 \mathrm{~Hz}, 2 \mathrm{H}), 7.22(\mathrm{~d}$, $J=8.6 \mathrm{~Hz}, 1 \mathrm{H}), 7.05(\mathrm{t}, J=7.5 \mathrm{~Hz}, 1 \mathrm{H}), 5.51(\mathrm{dd}, J=13.4,3.3 \mathrm{~Hz}, 1 \mathrm{H}), 3.97(\mathrm{dd}, J=18.0,3.4 \mathrm{~Hz}$, 1H), 3.87 (s, 3H), 3.53-3.40 (m, 1H), $2.44(\mathrm{~s}, 3 \mathrm{H}) ;{ }^{13} \mathrm{C} \mathrm{NMR}\left(100 \mathrm{MHz}, \mathrm{CDCl}_{3}\right) \delta 163.9,152.3,143.1$, 141.0, 139.5, 138.0, 129.5, 129.4, 129.0, 128.8, 126.7, 126.6, 120.6, 119.9, 117.3, 115.4, 110.0, 81.2, 37.7, 32.2, 21.6; IR (film): 3065, 3027, 2908, 1610, 1551, 1298, 1243, 1148, 1086, $955 \mathrm{~cm}^{-1}$; HRMS (EI): calcd for $\mathrm{C}_{25} \mathrm{H}_{22} \mathrm{~N}_{2} \mathrm{O}_{3} \mathrm{~S}\left([\mathrm{M}]^{+}\right)$: 430.1351, Found: 430.1348 .

4-methyl- $N$-(5-methyl-2-(4-nitrophenyl)-2,3-dihydropyrano[3,2-b]indol-4(5H)-ylidene)benzenesulfonamide (5e):<smiles>Cn1c2c(c3ccccc31)OC(c1ccc([N+](=O)[O-])cc1)C/C2=N/[As]</smiles>

Yellow solid (20.9 mg, 22\%); M.p. 193-194 ${ }^{0} \mathrm{C} ;{ }^{1} \mathrm{H}$ NMR (400 MHz, $\left.\mathrm{CDCl}_{3}\right) \delta 8.02(\mathrm{~d}, J=8.7 \mathrm{~Hz}, 2 \mathrm{H})$, $7.67(\mathrm{~d}, J=7.6 \mathrm{~Hz}, 1 \mathrm{H}), 7.62(\mathrm{~d}, J=8.2 \mathrm{~Hz}, 2 \mathrm{H}), 7.43(\mathrm{t}, J=7.7 \mathrm{~Hz}, 1 \mathrm{H}), 7.30(\mathrm{~d}, J=8.1 \mathrm{~Hz}, 2 \mathrm{H})$, $7.15(\mathrm{~d}, J=8.6 \mathrm{~Hz}, 2 \mathrm{H}), 6.89-6.77(\mathrm{~m}, 2 \mathrm{H}), 6.26(\mathrm{~d}, J=8.2 \mathrm{~Hz}, 1 \mathrm{H}), 5.48-5.43(\mathrm{~m}, 1 \mathrm{H}), 4.77(\mathrm{~s}, 1 \mathrm{H})$, $2.44(\mathrm{~s}, 3 \mathrm{H}), 2.25$ (s, 3H); ${ }^{13} \mathrm{CNMR}\left(100 \mathrm{MHz}, \mathrm{CDCl}_{3}\right) \delta$ 197.7, 159.8, 147.4, 144.4, 143.1, 139.0, 
136.7, 131.6, 129.7, 128.7, 127.4, 124.9, 123.3, 118.9, 118.5, 107.6, 106.2, 87.7, 58.5, 29.0, 21.7; IR (film): 3104, 2925, 2833, 2256, 1714, 1614, 1520, 1346, 1164, $998 \mathrm{~cm}^{-1}$; HRMS (EI): calcd for $\mathrm{C}_{25} \mathrm{H}_{21} \mathrm{~N}_{3} \mathrm{O}_{5} \mathrm{~S}\left([\mathrm{M}]^{+}\right): 475.1202$, Found: 475.1206 .

$N$-(2-(4-methoxyphenyl)-5-methyl-2,3-dihydropyrano[3,2-b]indol-4(5H)-ylidene)-4methylbenzenesulfonamide (5f):

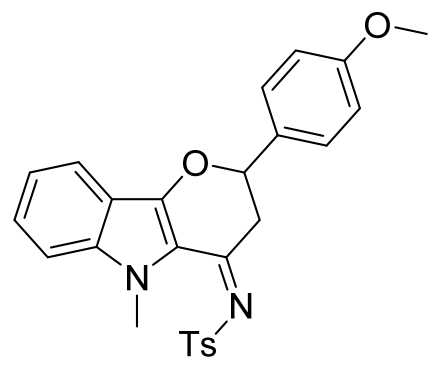

Yellow solid (24.8 mg, 27\%); M.p. 227-228 ${ }^{0} \mathrm{C}$; ${ }^{1} \mathrm{H}$ NMR (400 MHz, $\left.\mathrm{CDCl}_{3}\right) \delta$ 7.67-7.57 (m, 3H), 7.44 $-7.34(\mathrm{~m}, 1 \mathrm{H}), 7.32-7.23(\mathrm{~m}, 2 \mathrm{H}), 6.86(\mathrm{~d}, J=8.6 \mathrm{~Hz}, 2 \mathrm{H}), 6.79-6.72(\mathrm{~m}, 2 \mathrm{H}), 6.69-6.63(\mathrm{~m}, 2 \mathrm{H})$, $6.23(\mathrm{~d}, J=8.3 \mathrm{~Hz}, 1 \mathrm{H}), 5.43(\mathrm{dd}, J=4.5,2.1 \mathrm{~Hz}, 1 \mathrm{H}), 4.66(\mathrm{t}, J=2.4 \mathrm{~Hz}, 1 \mathrm{H}), 3.71(\mathrm{~s}, 3 \mathrm{H}), 2.42(\mathrm{~s}$, $3 \mathrm{H}), 2.14(\mathrm{~s}, 3 \mathrm{H}) ;{ }^{13} \mathrm{C} \mathrm{NMR}\left(100 \mathrm{MHz}, \mathrm{CDCl}_{3}\right) \delta 198.5,159.8,159.0,144.0,138.5,137.0,130.2$, 129.6, 129.0, 127.4, 127.2, 124.8, 118.8, 117.7, 113.4, 108.1, 107.3, 87.7, 58.2, 55.2, 28.9, 21.6; IR (film): 2964, 2928, 2839, 2259, 1869, 1714, 1613, 1514, 1487, $1040 \mathrm{~cm}^{-1}$; HRMS (EI): calcd for $\mathrm{C}_{26} \mathrm{H}_{24} \mathrm{~N}_{2} \mathrm{O}_{4} \mathrm{~S}\left([\mathrm{M}]^{+}\right): 460.1457$, Found: 460.1459 .

10-methylchromeno[3,2-b]indol-11(10H)-one (6a):

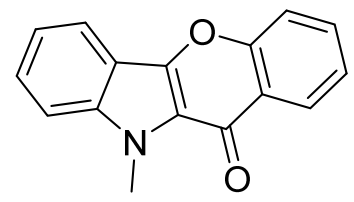

White solid (46.8 mg, 94\%); M.p. $192-193{ }^{\circ} \mathrm{C} ;{ }^{1} \mathrm{H}$ NMR (400 MHz, $\left.\mathrm{CDCl}_{3}\right) \delta 8.34$ (d, $\left.J=8.0 \mathrm{~Hz}, 1 \mathrm{H}\right)$, $7.90(\mathrm{dd}, J=8.1,0.7 \mathrm{~Hz}, 1 \mathrm{H}), 7.66-7.50(\mathrm{~m}, 2 \mathrm{H}), 7.46-7.40(\mathrm{~m}, 1 \mathrm{H}), 7.37-7.29(\mathrm{~m}, 2 \mathrm{H}), 7.19-7.12$ (m, 1H), $4.18(\mathrm{~d}, J=0.9 \mathrm{~Hz}, 3 \mathrm{H}) ;{ }^{13} \mathrm{C}$ NMR $\left(100 \mathrm{MHz}, \mathrm{CDCl}_{3}\right) \delta 170.5,155.4,144.7,138.4,132.6$, 128.1, 126.0, 124.3, 123.7, 120.5, 120.2, 120.0, 118.0, 114.8, 110.1, 31.3; IR (film): 1642, 1606, 1503, 1458, 1378, 1299, 1252, 956, 920, $754 \mathrm{~cm}^{-1}$; $\mathrm{HRMS}(\mathrm{ESI})$ : calcd for $\mathrm{C}_{16} \mathrm{H}_{11} \mathrm{NO}_{2}+\mathrm{H}^{+}\left([\mathrm{M}+\mathrm{H}]^{+}\right)$: 250.0863, Found: 250.0858 .

7-methoxy-10-methylchromeno[3,2-b]indol-11(10H)-one (6b):<smiles>COc1ccc2c(c1)c1oc3ccccc3c(=O)c1n2C</smiles>

White solid (50.8 mg, 91\%); M.p. $190-191{ }^{0} \mathrm{C} ;{ }^{1} \mathrm{H}$ NMR (400 MHz, $\left.\mathrm{CDCl}_{3}\right) \delta 8.40(\mathrm{dd}, J=8.0,1.6 \mathrm{~Hz}$, $1 \mathrm{H}), 7.70-7.63(\mathrm{~m}, 1 \mathrm{H}), 7.62-7.57(\mathrm{~m}, 1 \mathrm{H}), 7.43-7.37(\mathrm{~m}, 1 \mathrm{H}), 7.32-7.28(\mathrm{~m}, 2 \mathrm{H}), 7.18-7.13(\mathrm{~m}, 1 \mathrm{H})$, $4.22(\mathrm{~s}, 3 \mathrm{H}), 3.91(\mathrm{~s}, 3 \mathrm{H}) ;{ }^{13} \mathrm{C} \mathrm{NMR}\left(100 \mathrm{MHz}, \mathrm{CDCl}_{3}\right) \delta 170.4,155.3,154.2,144.1,134.1,132.5$, 126.1, 124.1, 123.6, 120.8, 120.0, 117.9, 114.4, 111.2, 99.4, 55.8, 31.3; IR (film): 1866, 1747, 1646, 
1556, 1516, 1417, 1309, 1259, 1205, $1052 \mathrm{~cm}^{-1}$; $\mathrm{HRMS}(\mathrm{ESI})$ : calcd for $\mathrm{C}_{17} \mathrm{H}_{13} \mathrm{NO}_{3}+\mathrm{H}^{+}\left([\mathrm{M}+\mathrm{H}]^{+}\right)$: 280.0968 , Found: 280.0965 .

7-fluoro-10-methylchromeno[3,2-b]indol-11(10H)-one (6c):<smiles>Cn1c2ccc(F)cc2c2oc3ccccc3c(=O)c21</smiles>

White solid (47.0 mg, 88\%); M.p. 224-225 ${ }^{\circ} \mathrm{C}$; ${ }^{1} \mathrm{H}$ NMR (400 MHz, $\left.\mathrm{CDCl}_{3}\right) \delta 8.40(\mathrm{dd}, J=8.0,1.6 \mathrm{~Hz}$, $1 \mathrm{H}), 7.73-7.66(\mathrm{~m}, 1 \mathrm{H}), 7.65-7.56(\mathrm{~m}, 2 \mathrm{H}), 7.46-7.33(\mathrm{~m}, 2 \mathrm{H}), 7.30-7.21(\mathrm{~m}, 1 \mathrm{H}), 4.25(\mathrm{~s}, 3 \mathrm{H}),{ }^{13} \mathrm{C}$ NMR $\left(100 \mathrm{MHz}, \mathrm{CDCl}_{3}\right) \delta 170.6,157.5(\mathrm{~d}, J=238.4 \mathrm{~Hz}), 155.4,144.2,135.0,132.9,126.1,124.1$, 123.8, 121.6, 118.1, 117.2 (d, $J=27.0 \mathrm{~Hz}), 114.5$ (d, $J=10.3 \mathrm{~Hz}), 111.4$ (d, $J=9.2 \mathrm{~Hz}), 104.5$ (d, $J=$ $24.7 \mathrm{~Hz}$ ), 31.5; IR (film): 1643, 1503, 1459, 1378, 1300, 1134, 1109, 1025, 953, $738 \mathrm{~cm}^{-1}$; HRMS (ESI): calcd for $\mathrm{C}_{16} \mathrm{H}_{10} \mathrm{FNO}_{2}+\mathrm{H}^{+}\left([\mathrm{M}+\mathrm{H}]^{+}\right)$: 268.0768, Found: 268.0774 . 


\section{Copies of ${ }^{1} \mathbf{H} \&{ }^{13} \mathrm{C}$ NMR Spectra}

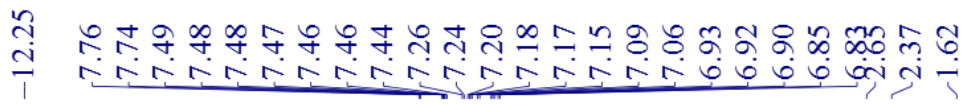
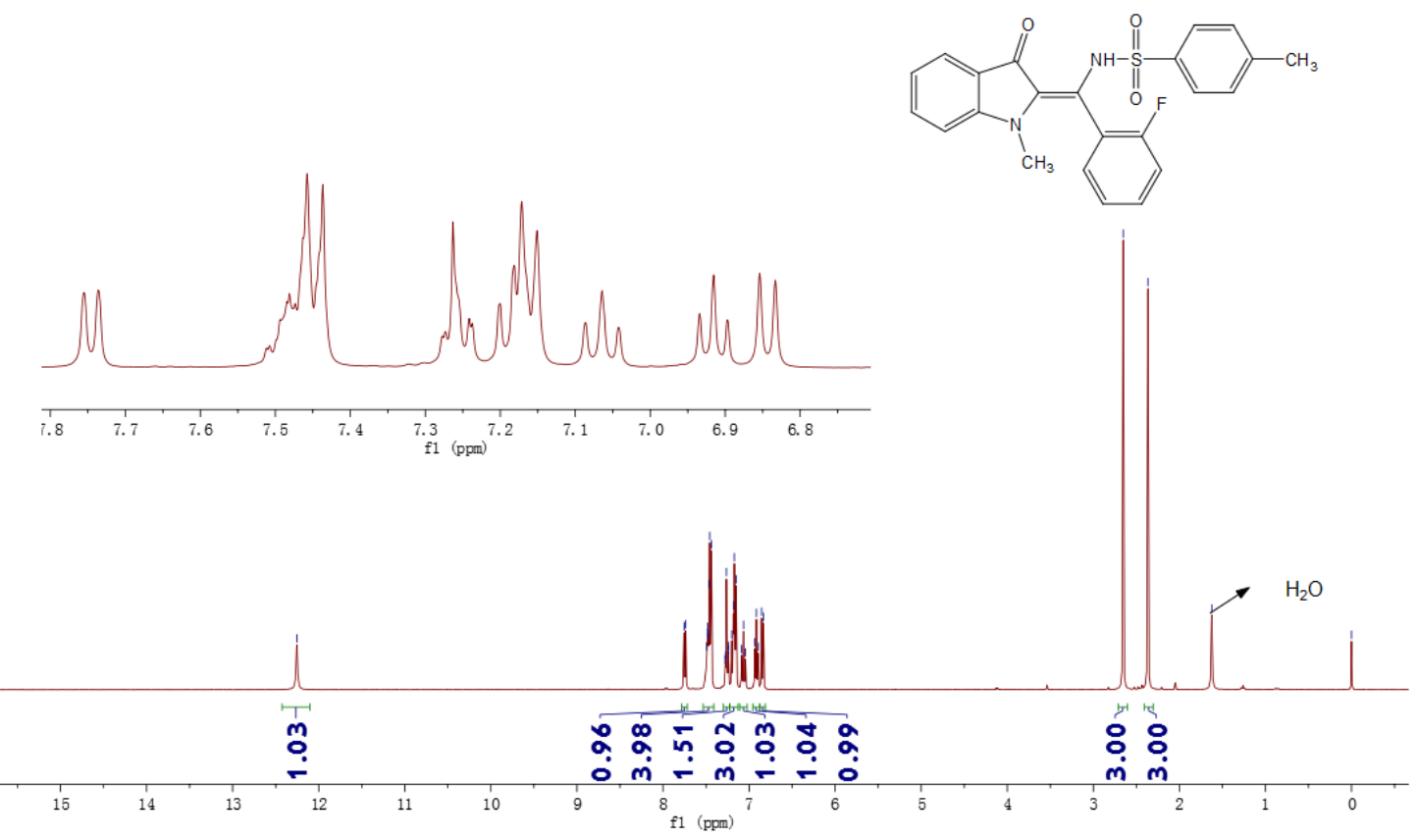

${ }^{1} \mathrm{H}$ NMR Spectrum for $\mathbf{3 a}\left(\mathrm{CDCl}_{3}, 400 \mathrm{MHz}\right)$

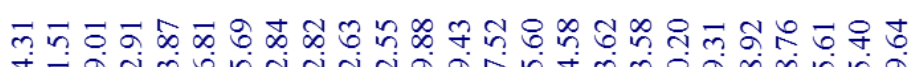
চ
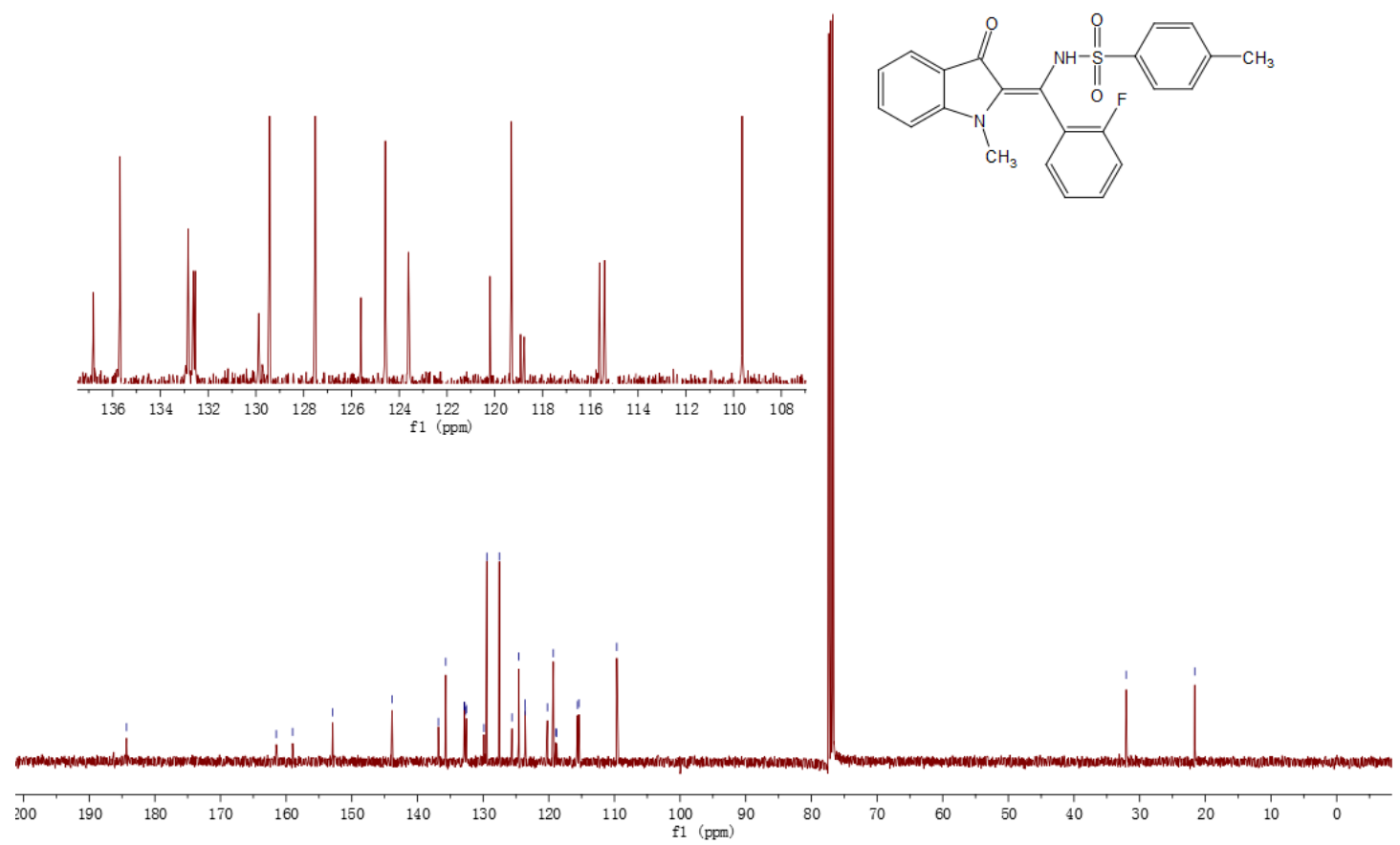

13

${ }^{13} \mathrm{CNMR}$ Spectrum for $3 \mathbf{a}\left(\mathrm{CDCl}_{3}, 100 \mathrm{MHz}\right)$ 

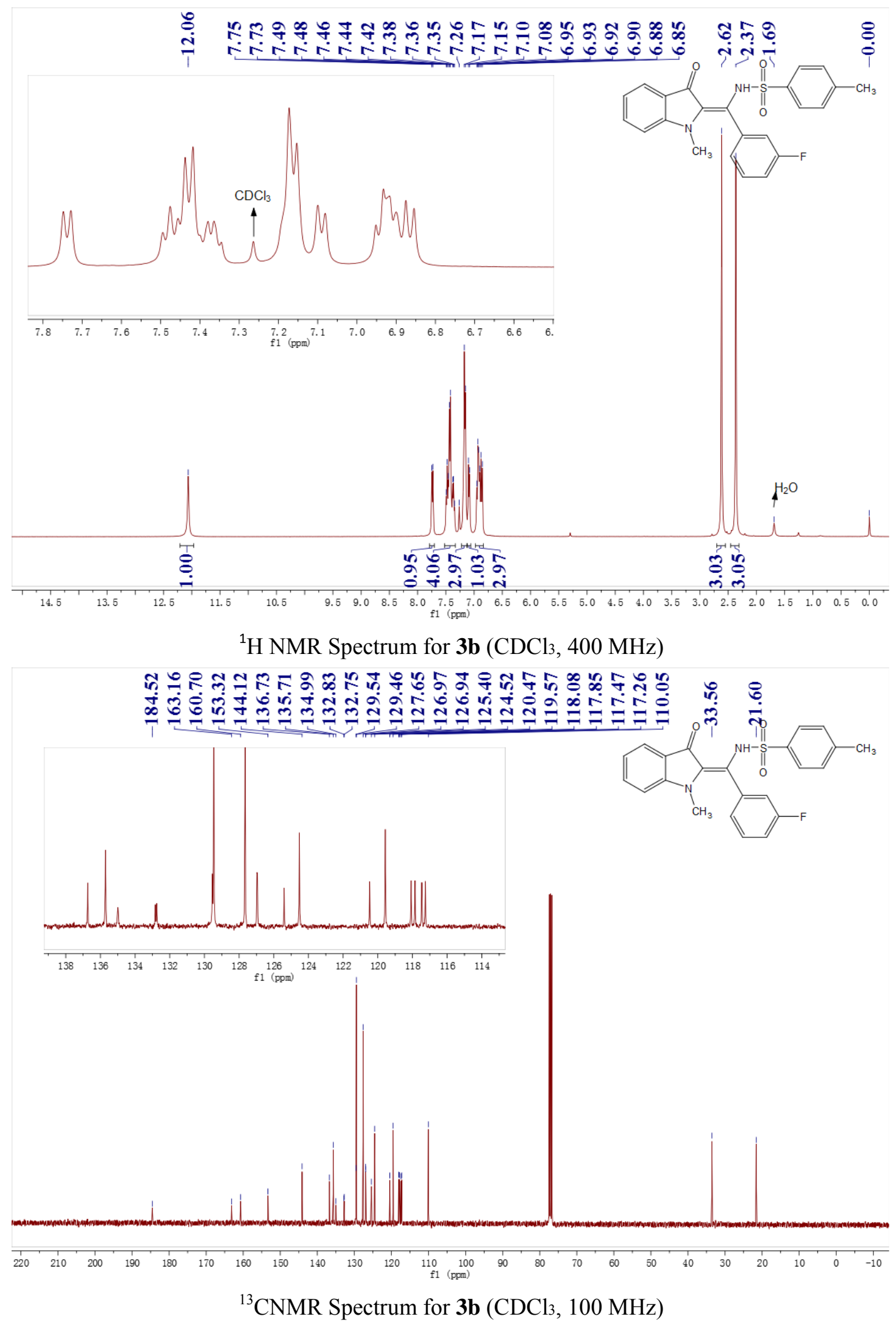


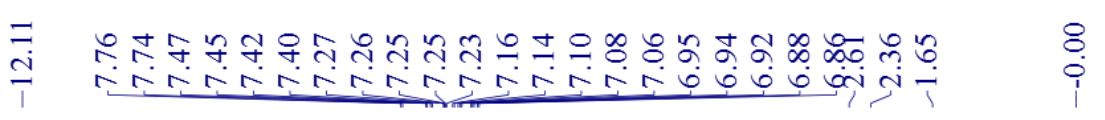
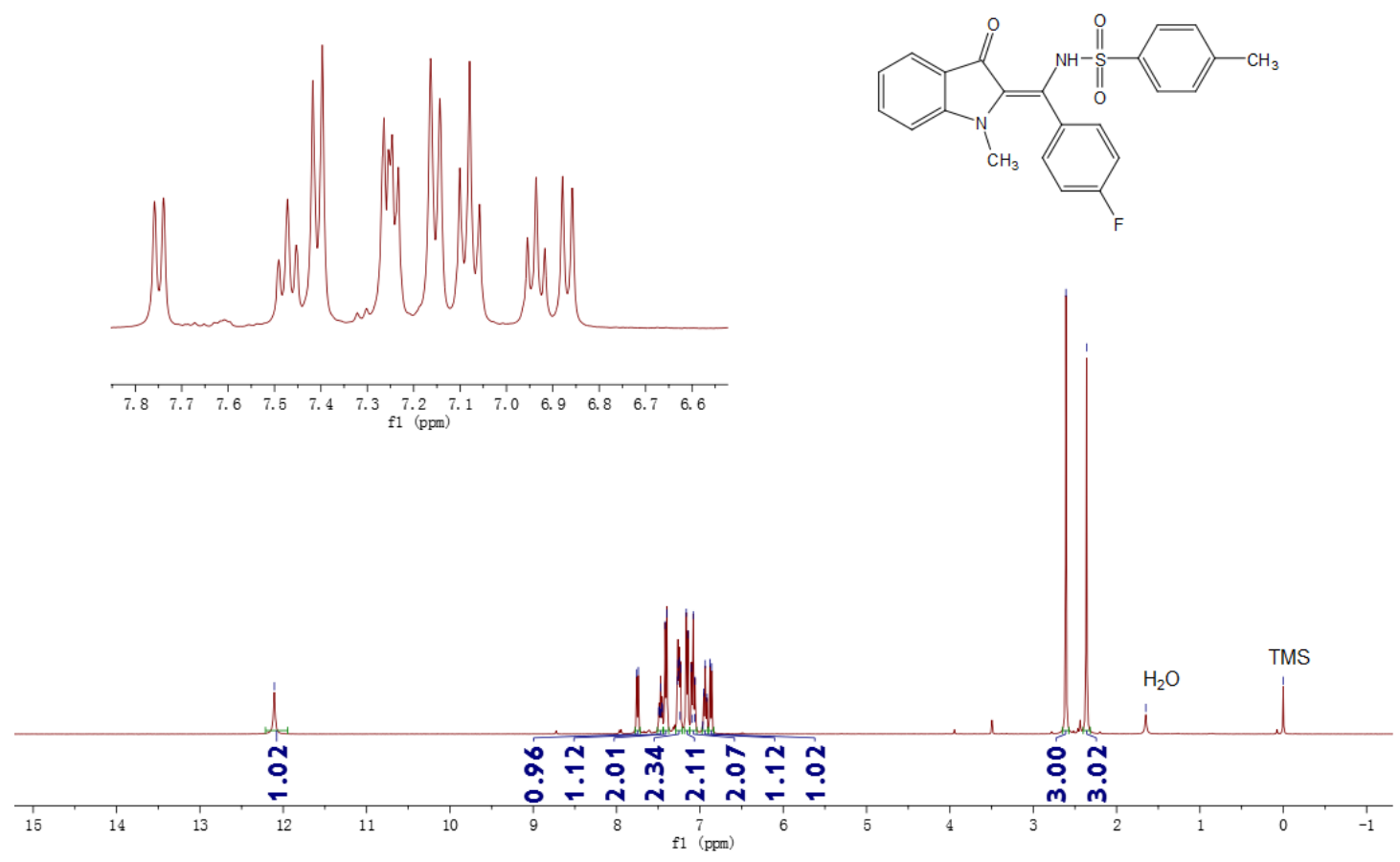

${ }^{1} \mathrm{H}$ NMR Spectrum for $3 \mathrm{c}\left(\mathrm{CDCl}_{3}, 400 \mathrm{MHz}\right)$

=

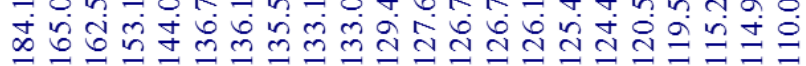

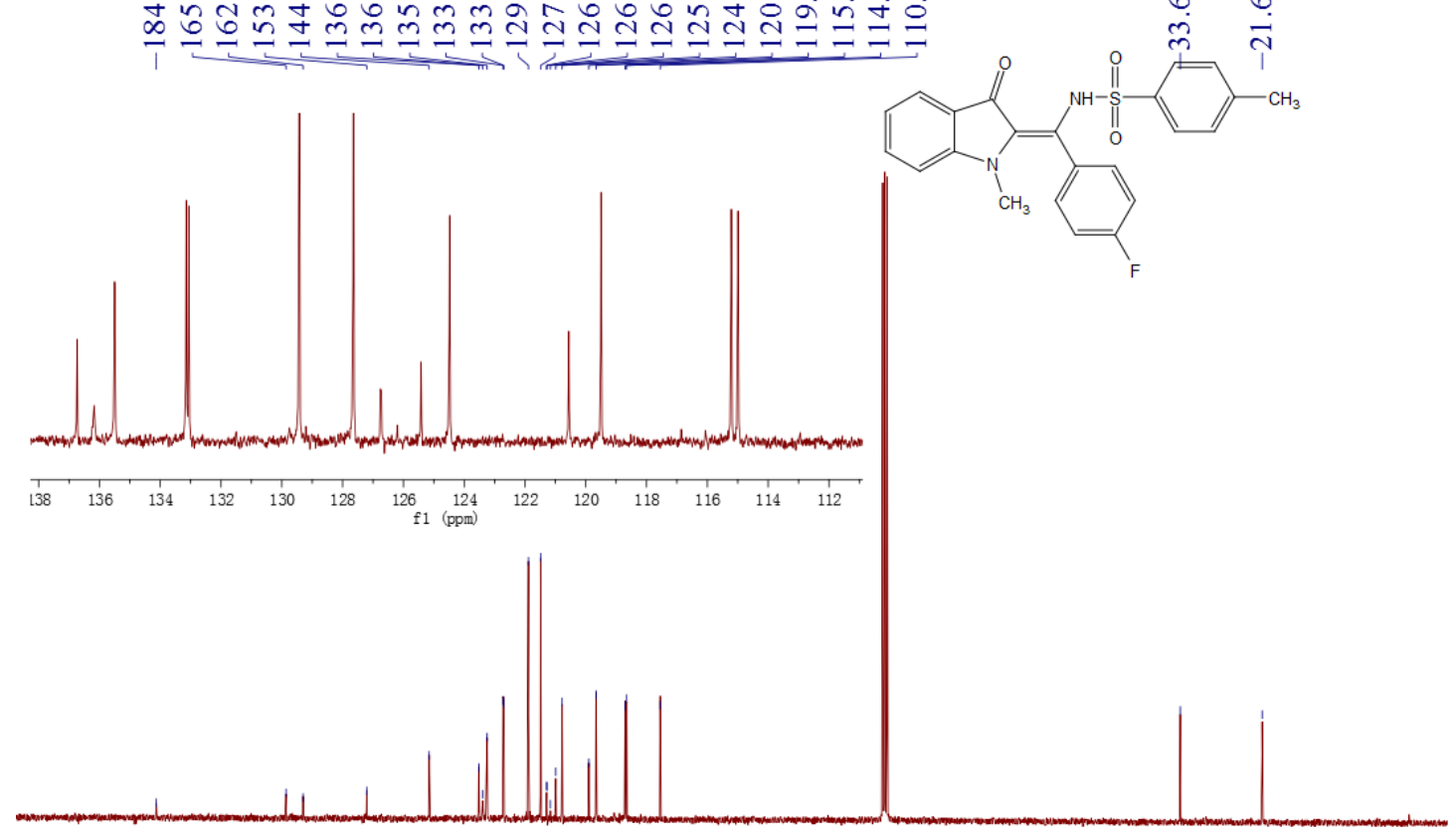

$200 \quad 190$

$180 \quad 170 \quad 160$

$150 \quad 140 \quad 130$

f1 100

${ }^{13} \mathrm{CNMR}$ Spectrum for $3 \mathrm{c}\left(\mathrm{CDCl}_{3}, 100 \mathrm{MHz}\right)$ 

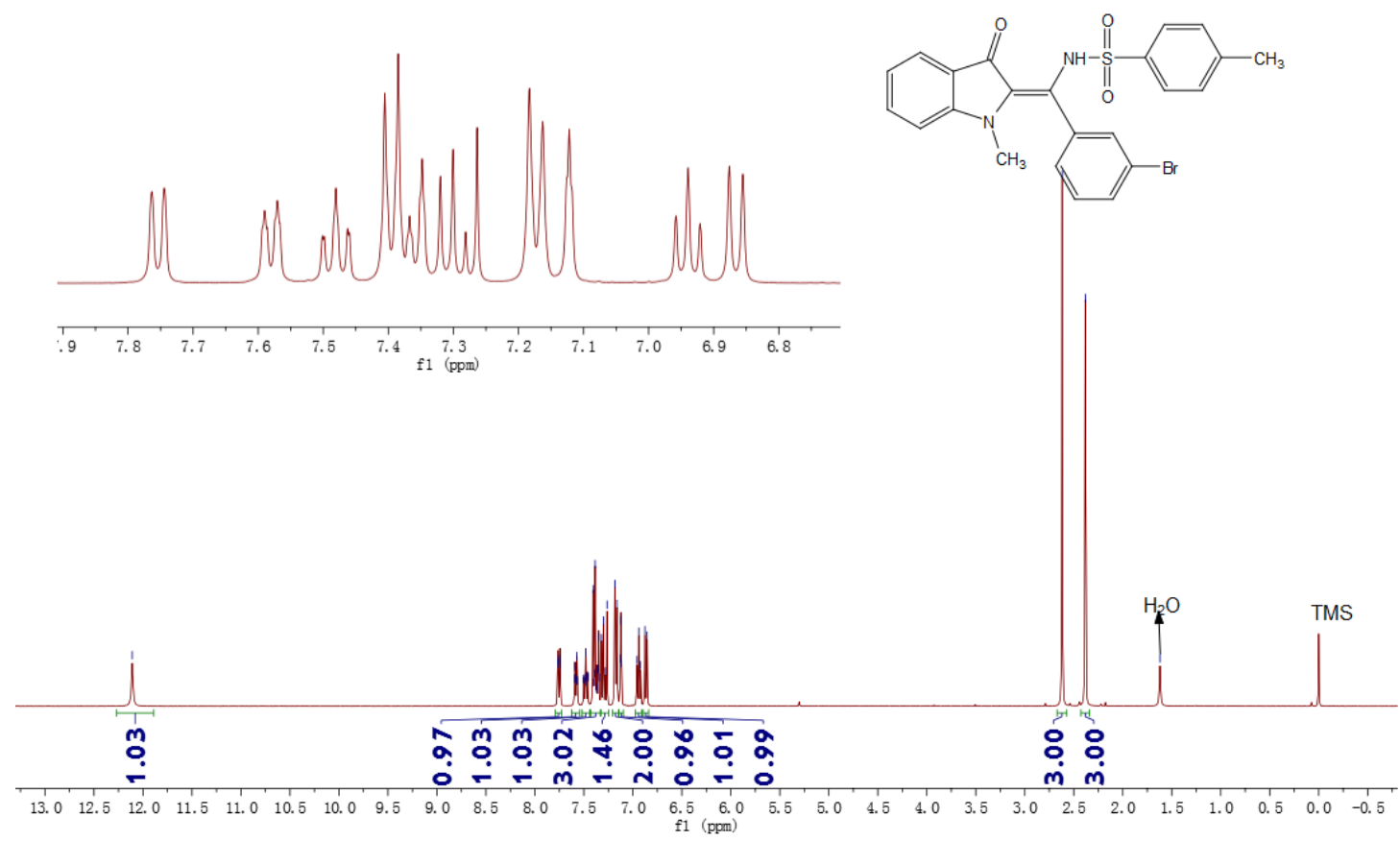

${ }^{1} \mathrm{H}$ NMR Spectrum for $\mathbf{3 d}\left(\mathrm{CDCl}_{3}, 400 \mathrm{MHz}\right)$

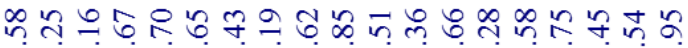

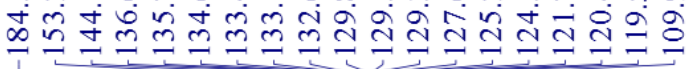
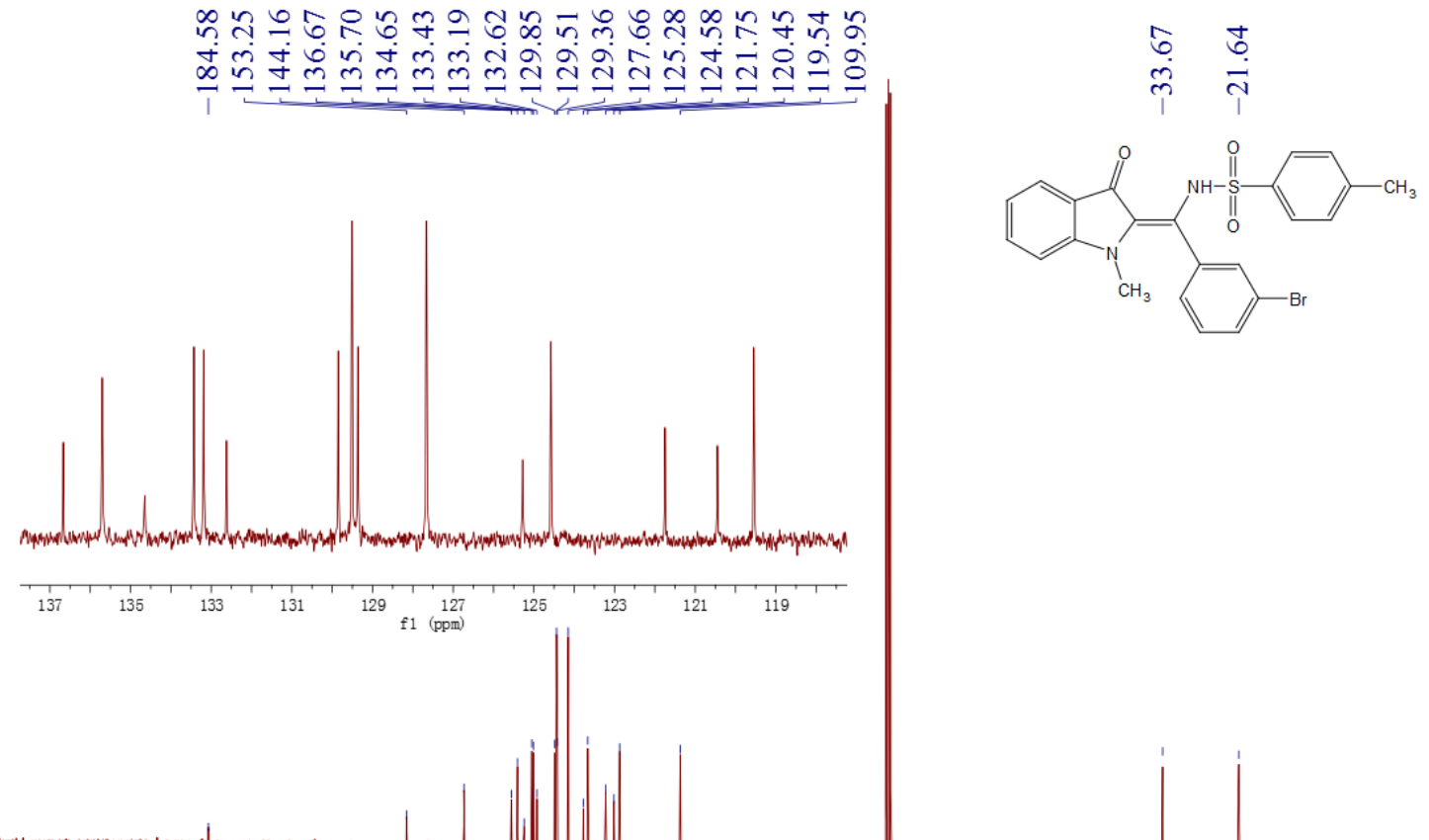

${ }^{13} \mathrm{CNMR}$ Spectrum for $3 \mathbf{d}\left(\mathrm{CDCl}_{3}, 100 \mathrm{MHz}\right)$ 


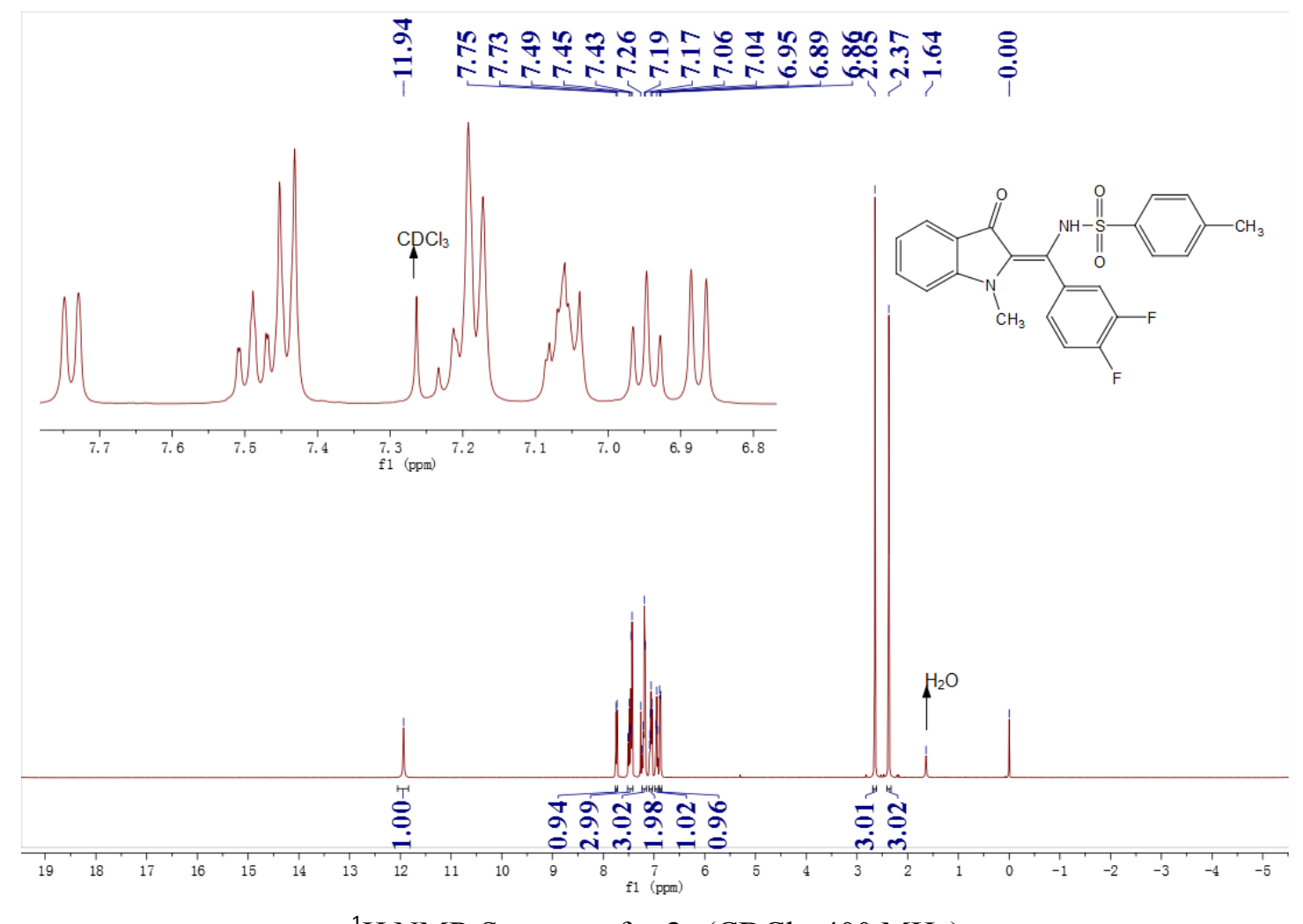

${ }^{1} \mathrm{H}$ NMR Spectrum for $3 \mathbf{e}\left(\mathrm{CDCl}_{3}, 400 \mathrm{MHz}\right)$
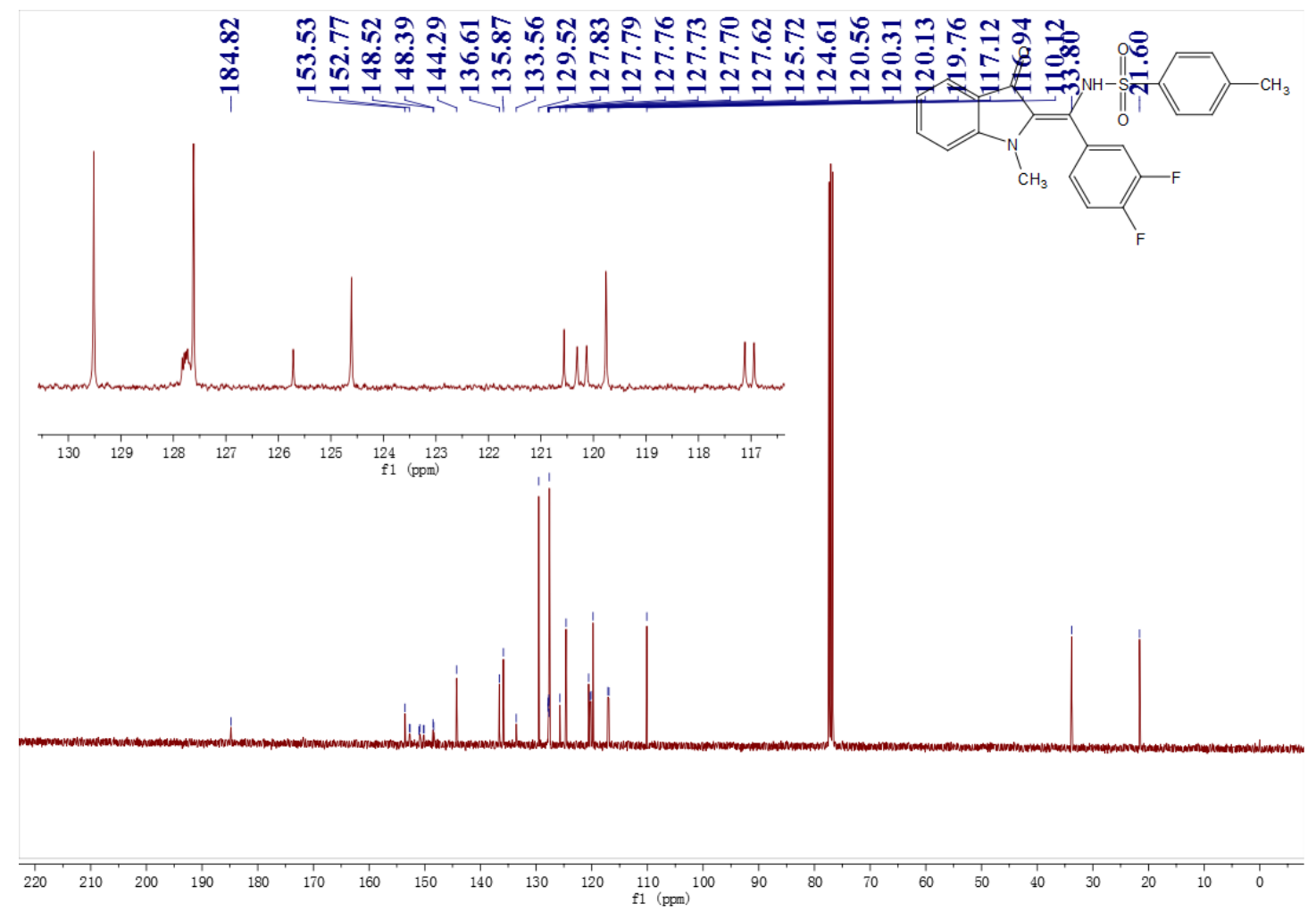

${ }^{13} \mathrm{CNMR}$ Spectrum for $3 \mathbf{e}\left(\mathrm{CDCl}_{3}, 100 \mathrm{MHz}\right)$ 


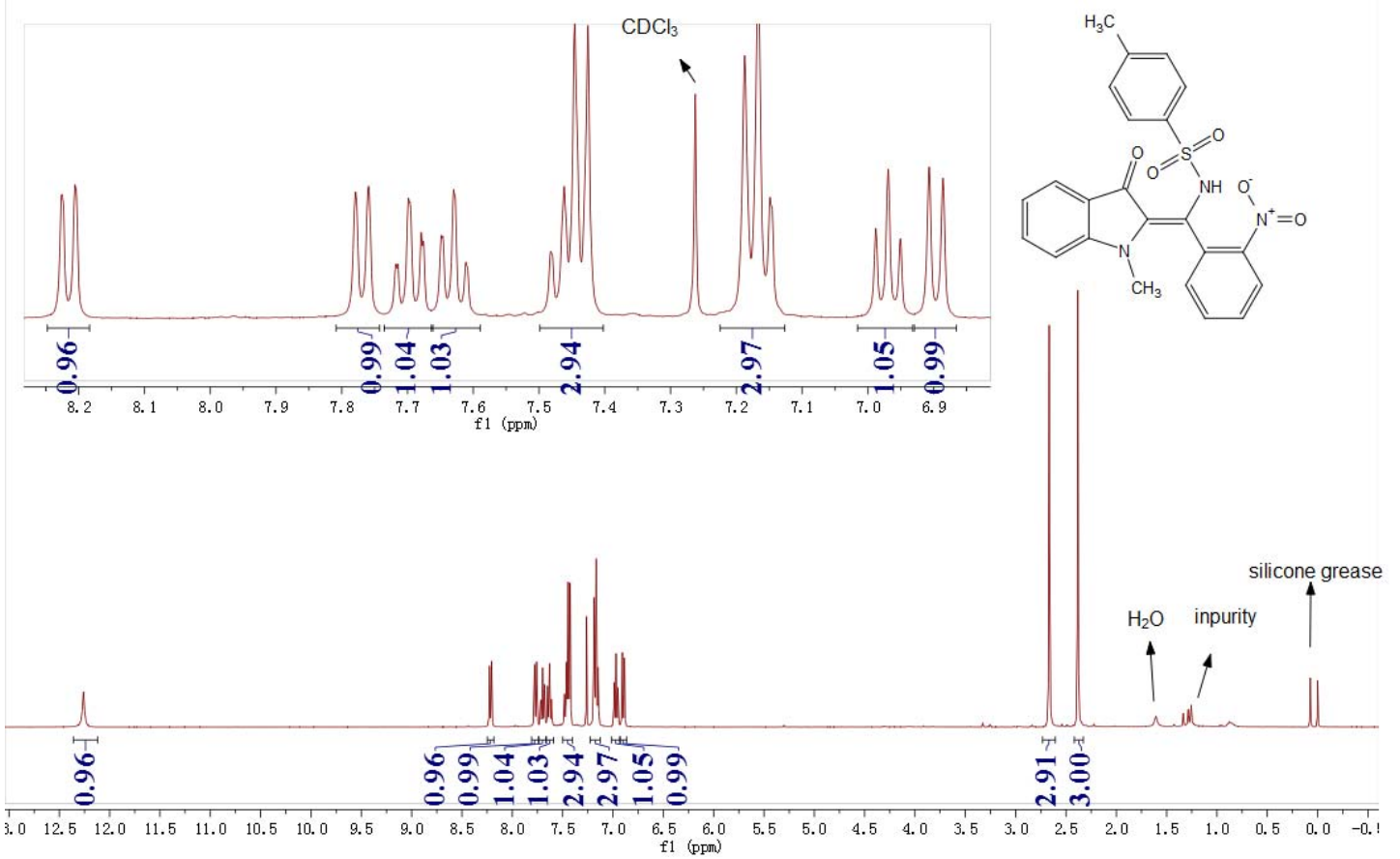

${ }^{1} \mathrm{H}$ NMR Spectrum for $\mathbf{3 f}\left(\mathrm{CDCl}_{3}, 400 \mathrm{MHz}\right)$

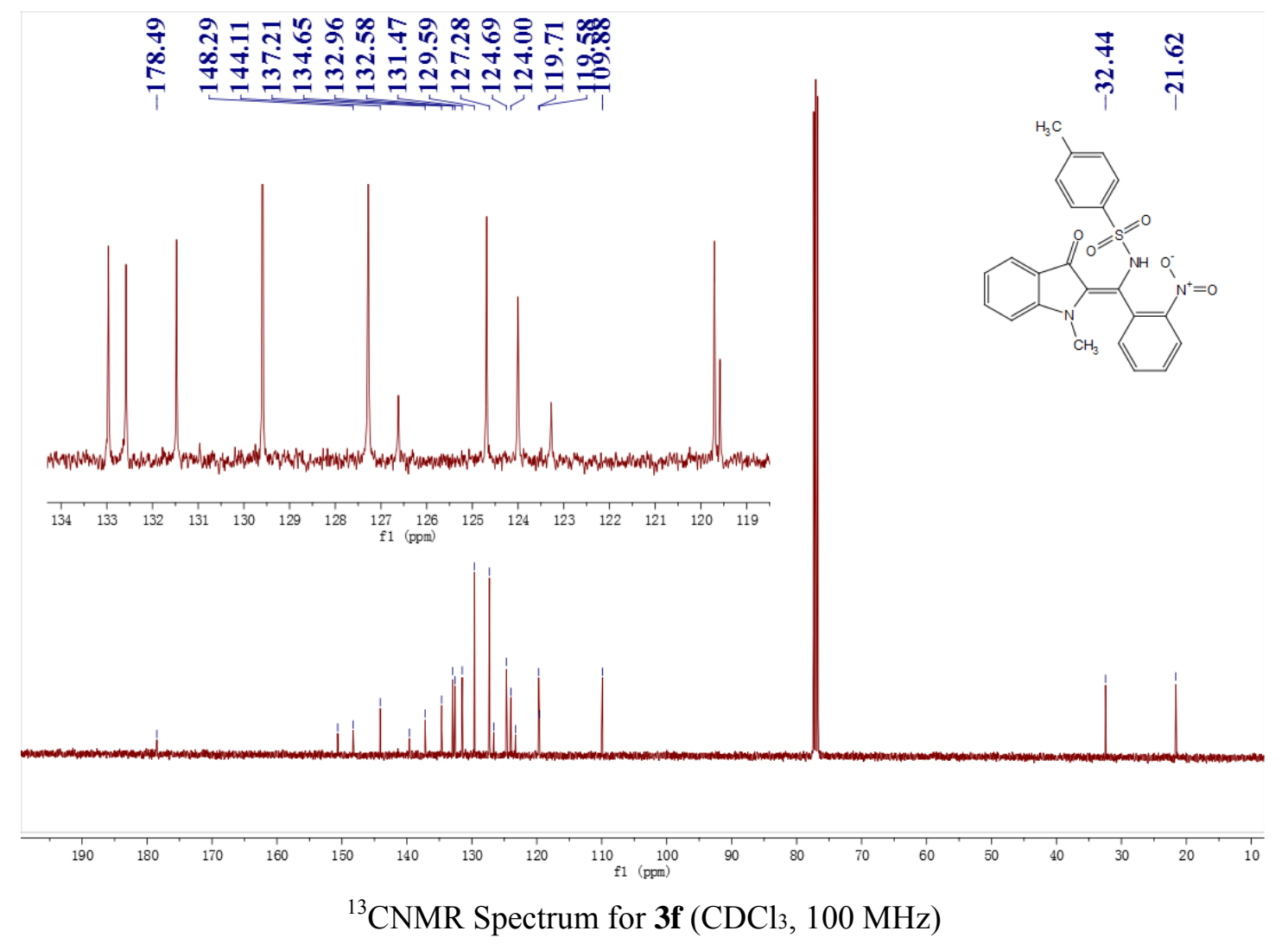




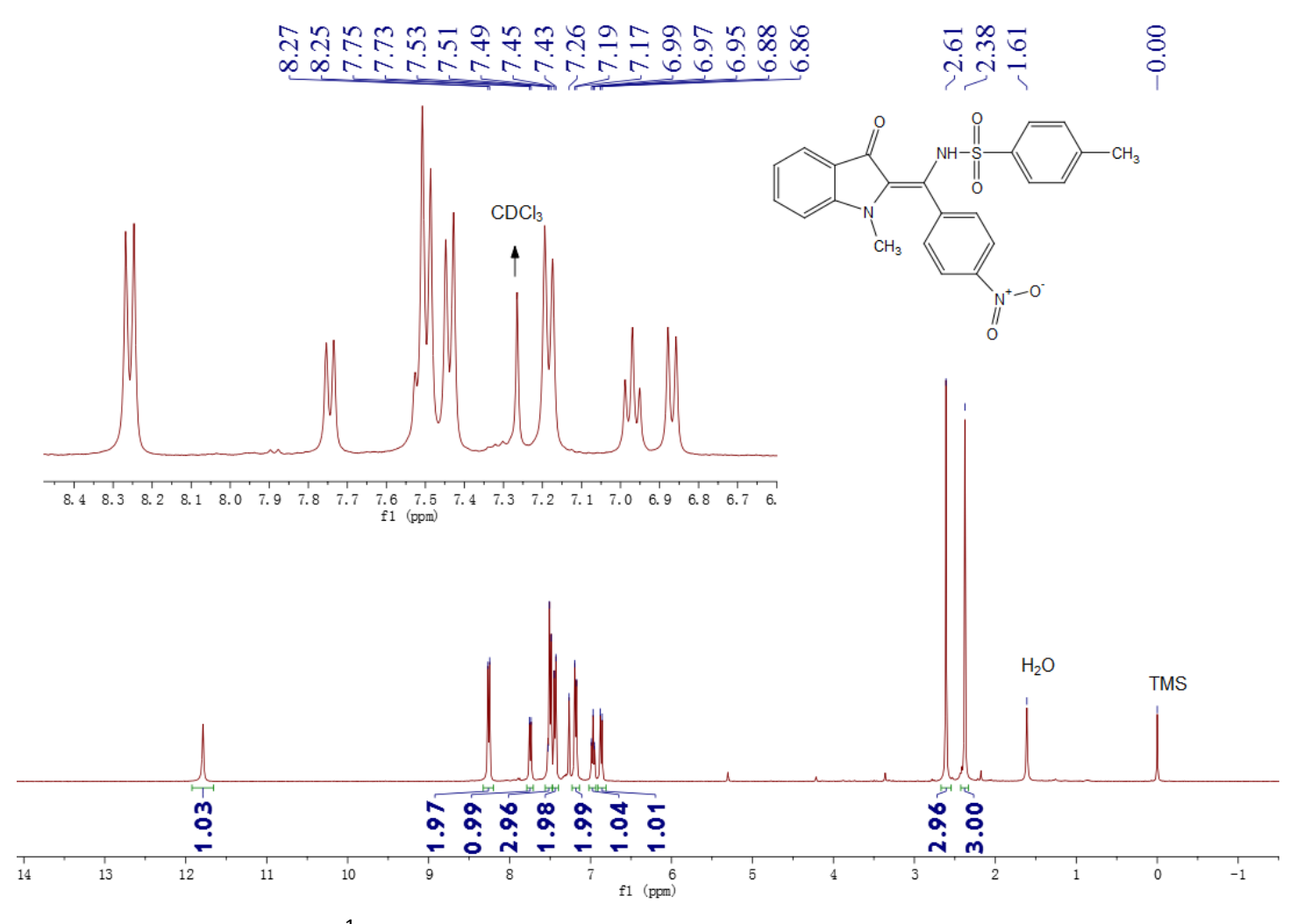

${ }^{1} \mathrm{H}$ NMR Spectrum for $\mathbf{3 g}\left(\mathrm{CDCl}_{3}, 400 \mathrm{MHz}\right)$

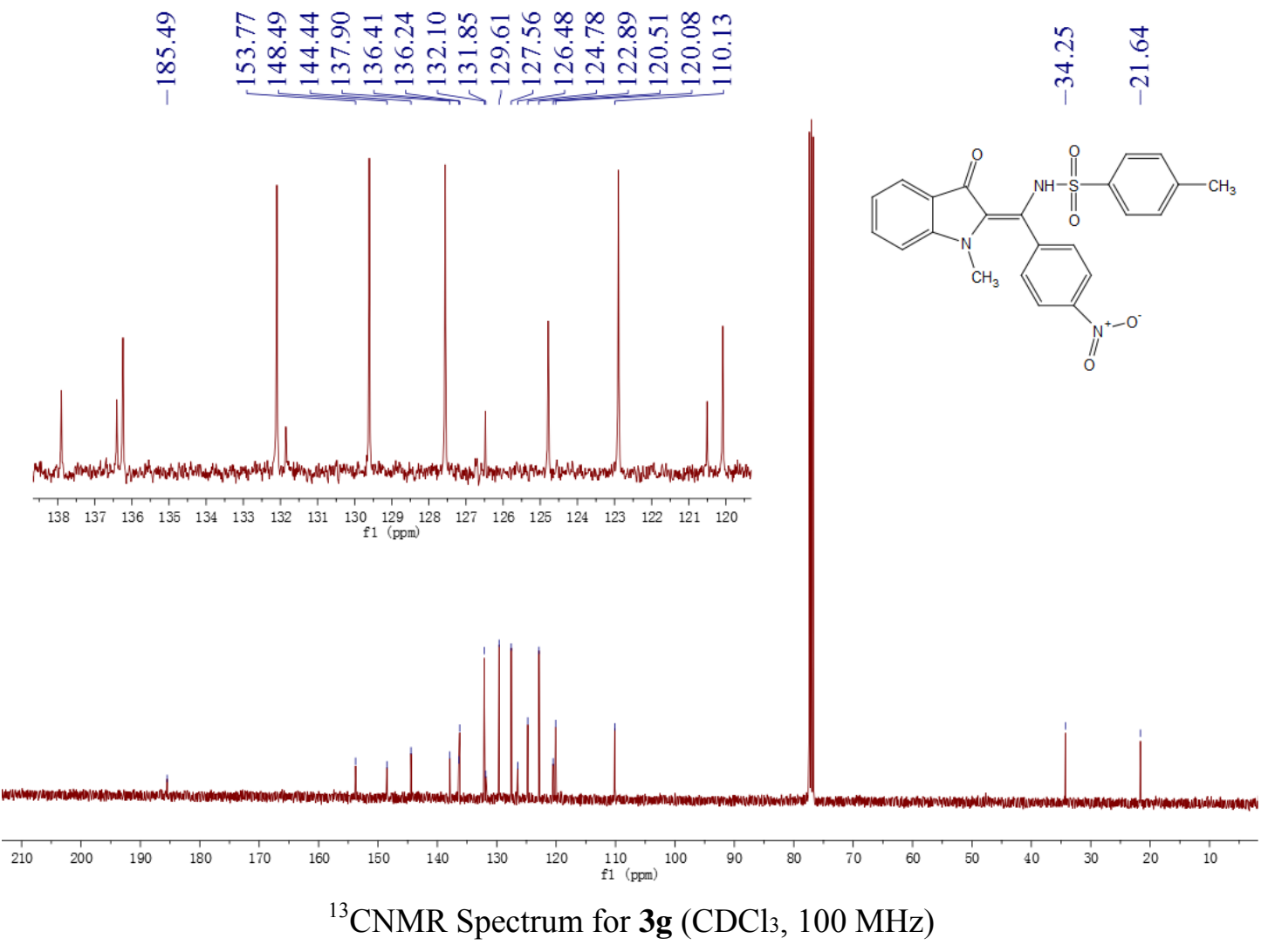




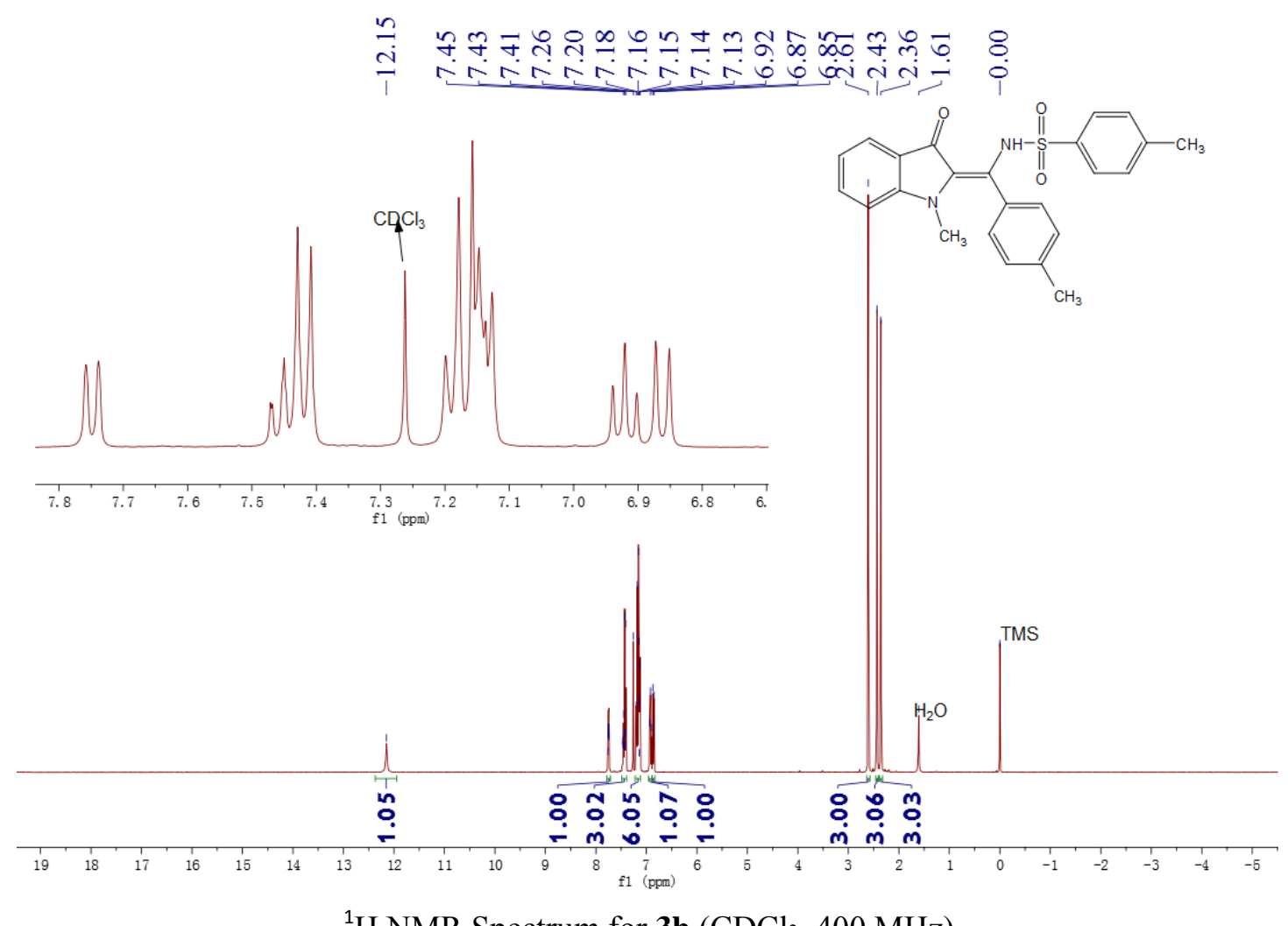

${ }^{1} \mathrm{H}$ NMR Spectrum for $\mathbf{3 h}\left(\mathrm{CDCl}_{3}, 400 \mathrm{MHz}\right)$

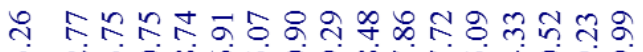

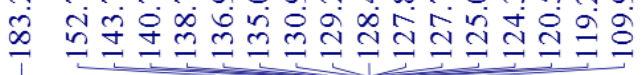

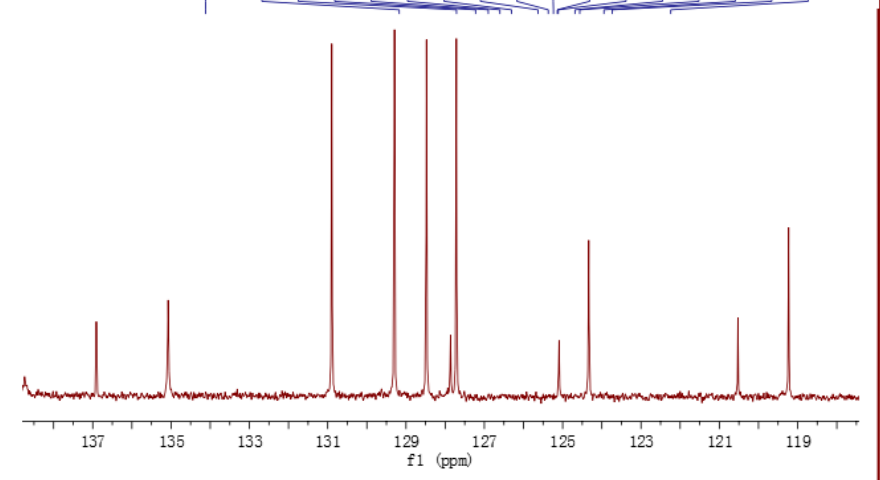

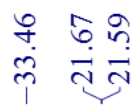
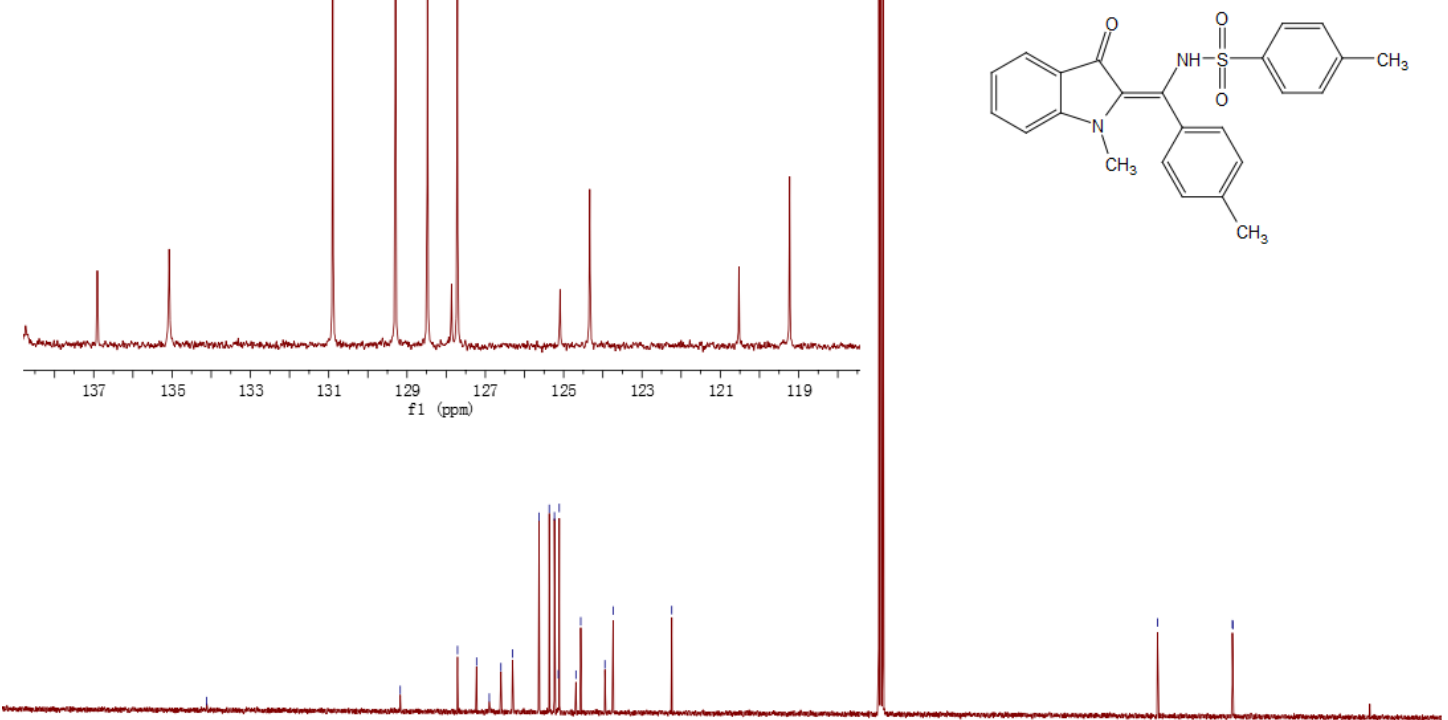

$\begin{array}{llllll}110 & 200 & 190 & 180 & 170 & 160\end{array}$

140

$110 \begin{array}{r}100 \\ \mathrm{f} 1(\mathrm{ppm})\end{array}$

${ }^{13} \mathrm{CNMR}$ Spectrum for $3 \mathbf{h}\left(\mathrm{CDCl}_{3}, 100 \mathrm{MHz}\right)$ 


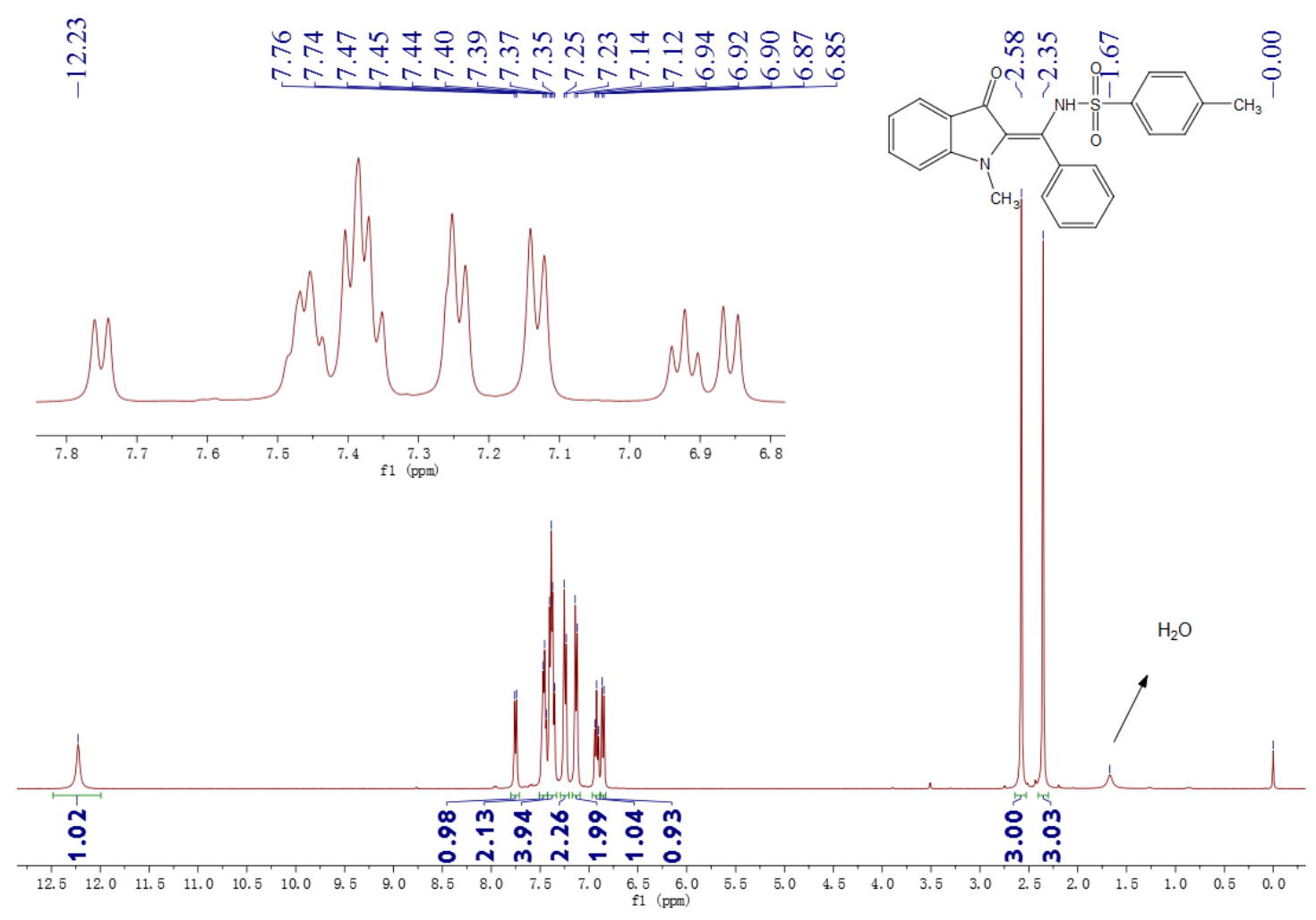

${ }^{1} \mathrm{H}$ NMR Spectrum for $\mathbf{3 i}\left(\mathrm{CDCl}_{3}, 400 \mathrm{MHz}\right)$
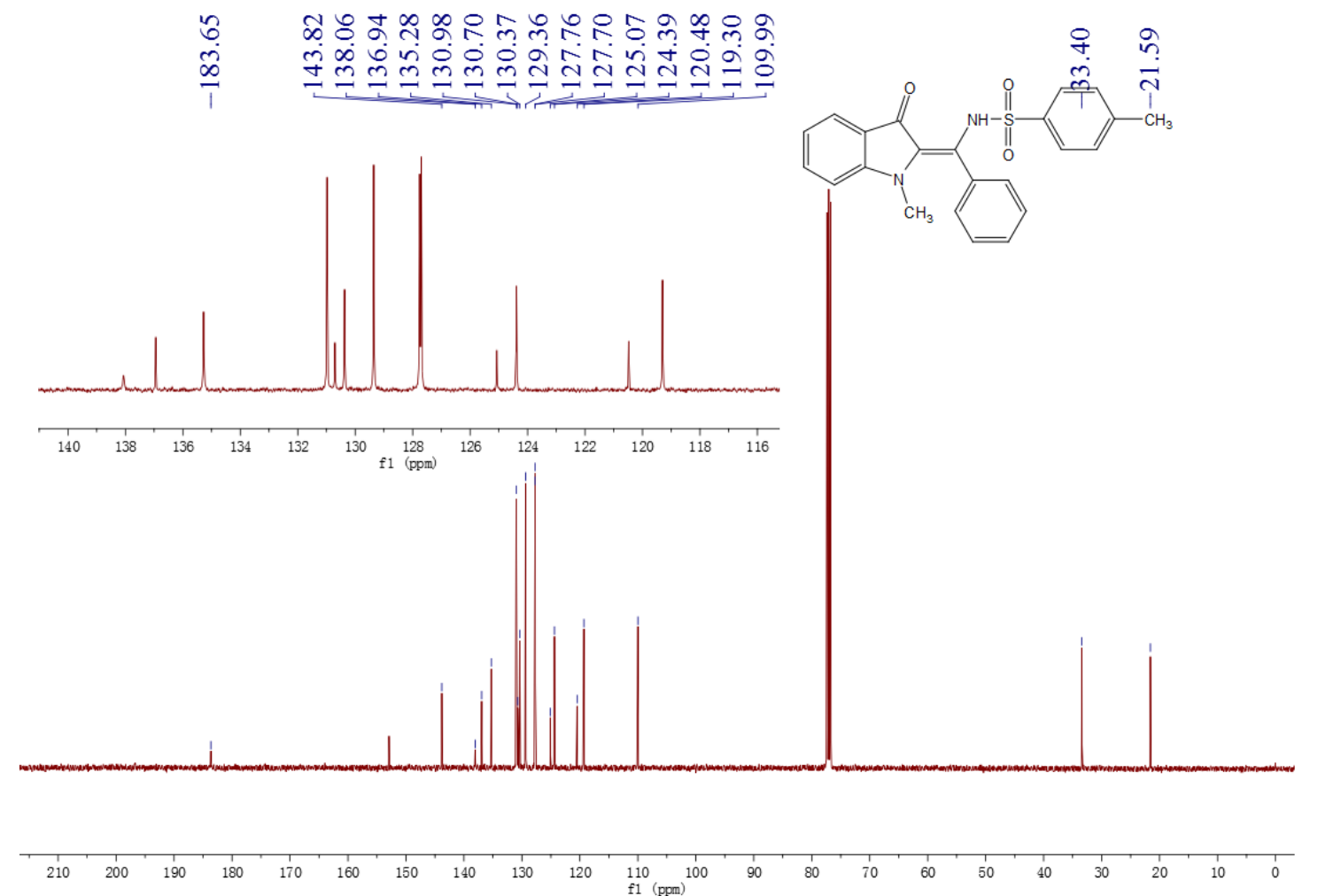

${ }^{13} \mathrm{CNMR}$ Spectrum for $3 \mathbf{i}\left(\mathrm{CDCl}_{3}, 100 \mathrm{MHz}\right)$ 


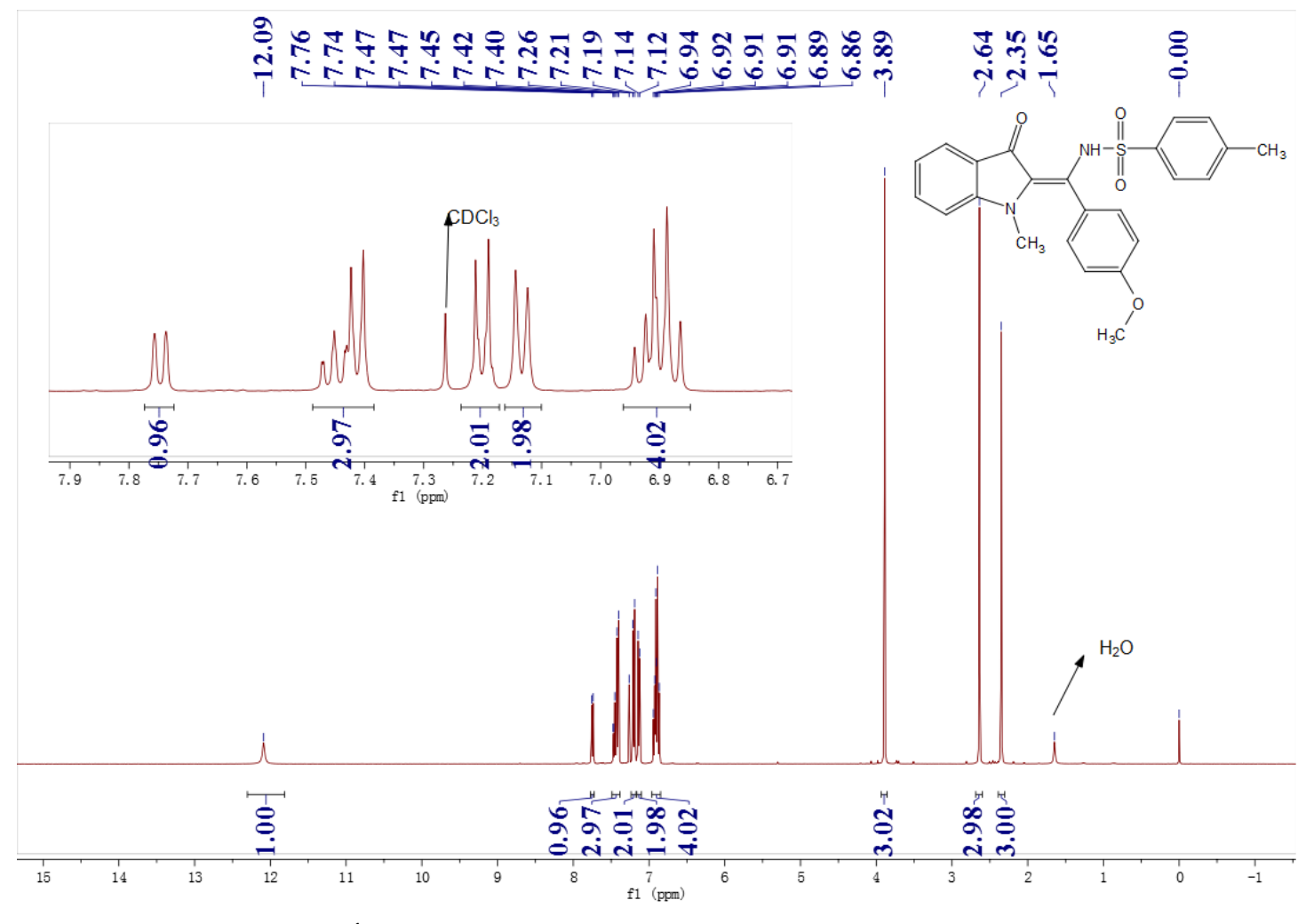

${ }^{1} \mathrm{H}$ NMR Spectrum for $3 \mathbf{j}\left(\mathrm{CDCl}_{3}, 400 \mathrm{MHz}\right)$

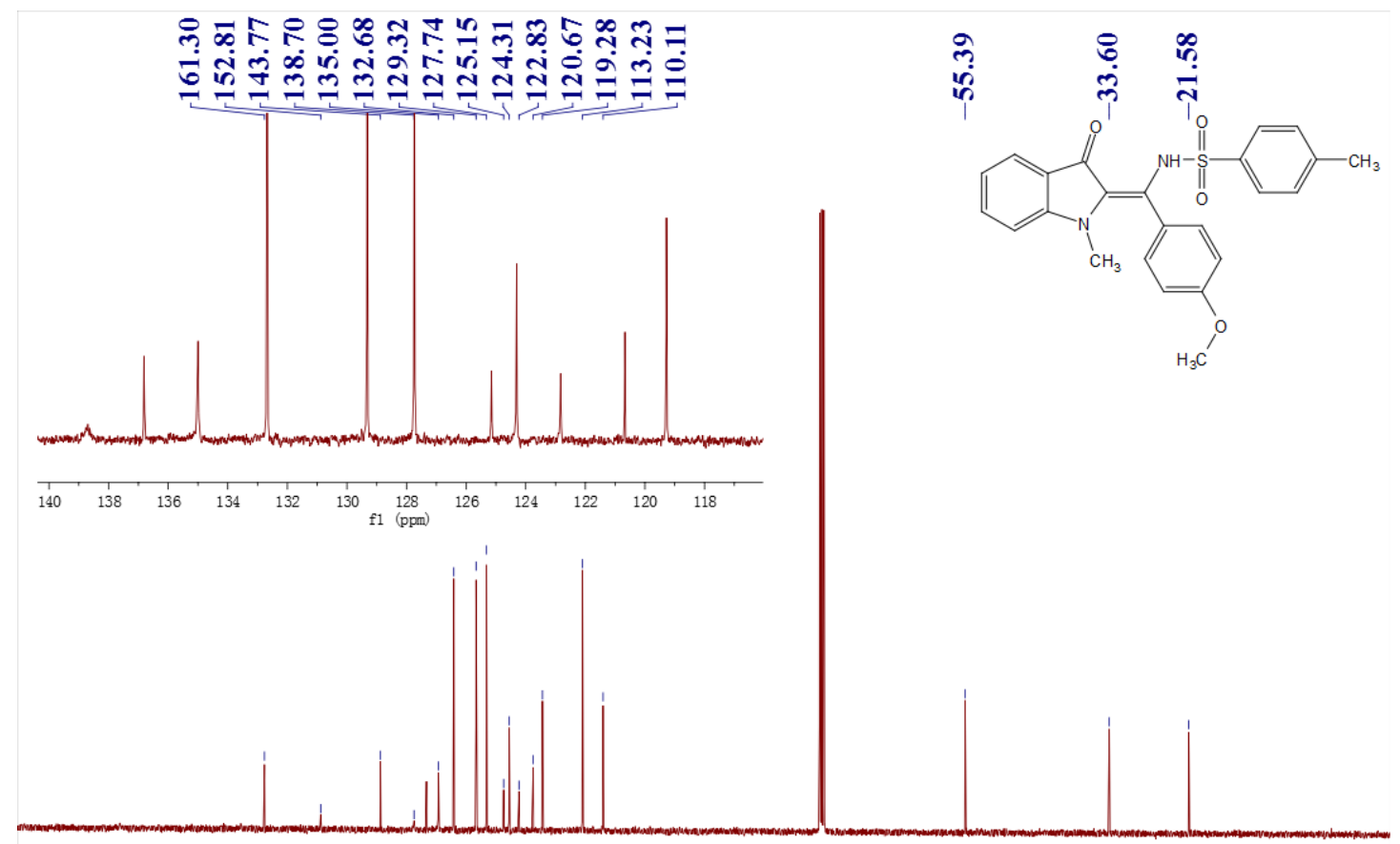

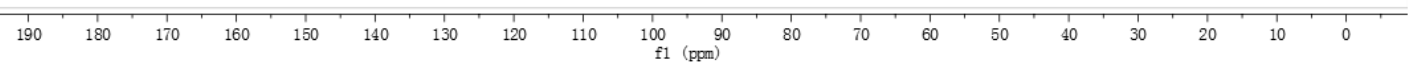

${ }^{13} \mathrm{CNMR}$ Spectrum for $\mathbf{3} \mathbf{j}\left(\mathrm{CDCl}_{3}, 100 \mathrm{MHz}\right)$ 

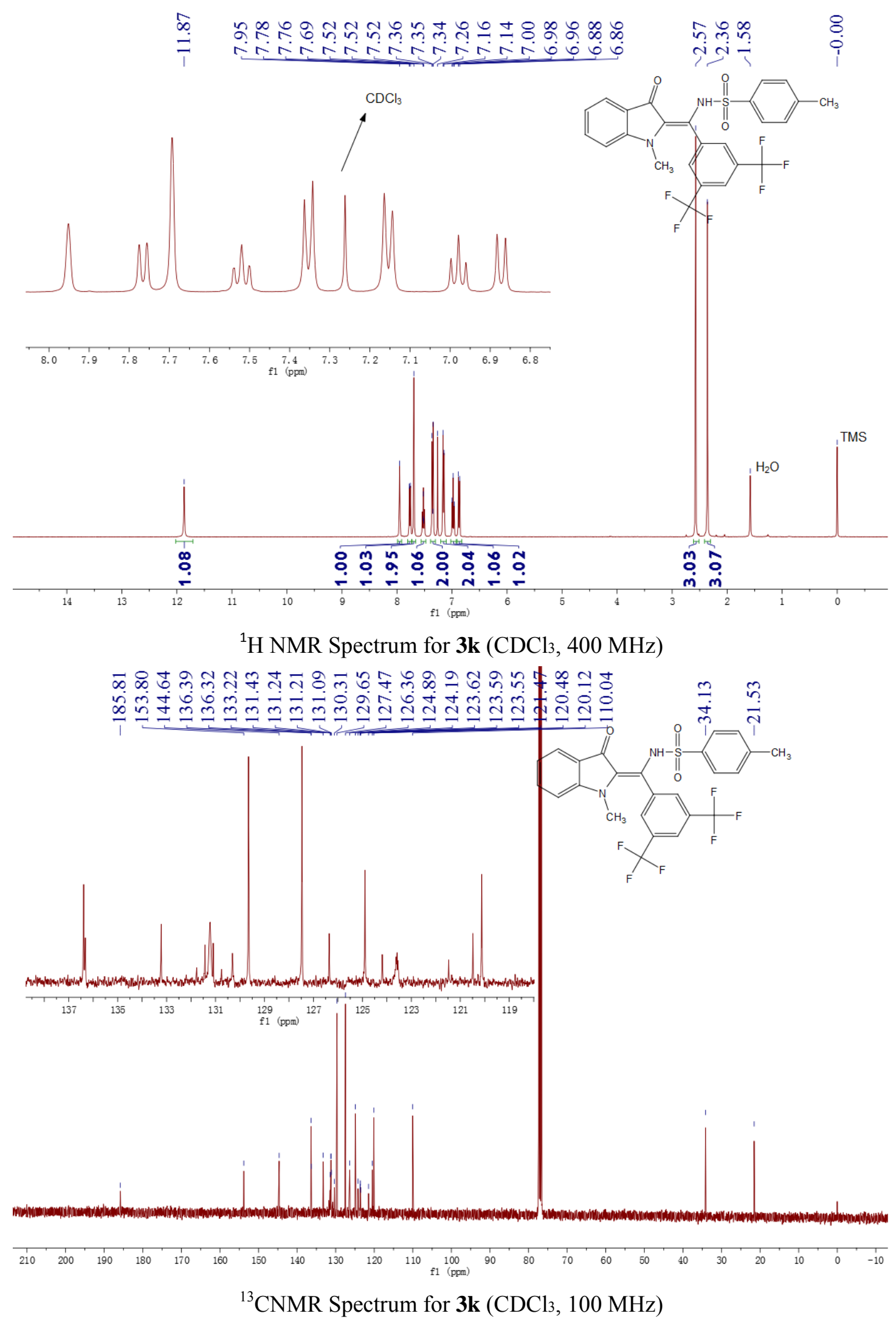


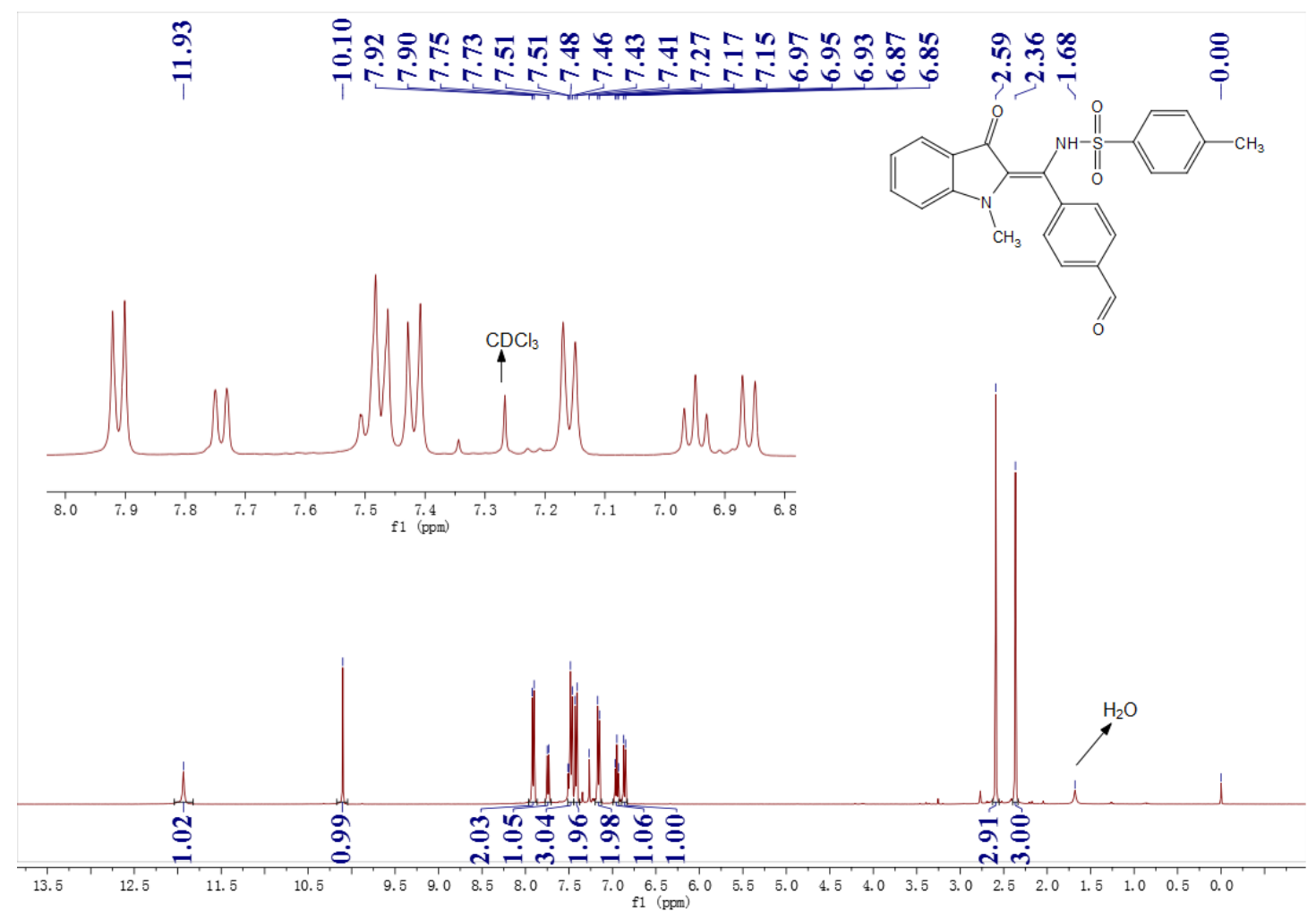

${ }^{1} \mathrm{H}$ NMR Spectrum for 31 ( $\left.\mathrm{CDCl}_{3}, 400 \mathrm{MHz}\right)$
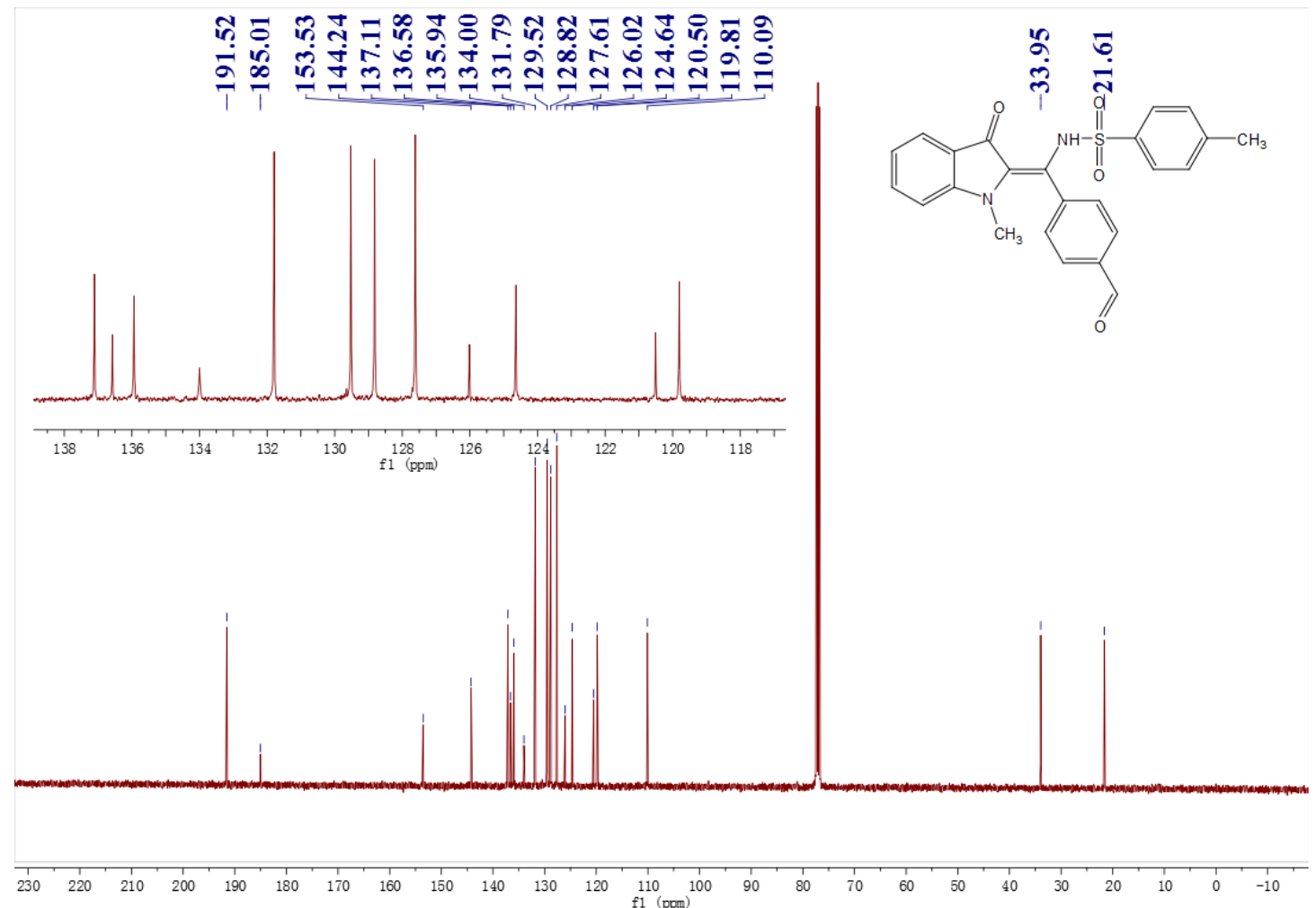

${ }^{13} \mathrm{CNMR}$ Spectrum for $3 \mathbf{l}\left(\mathrm{CDCl}_{3}, 100 \mathrm{MHz}\right)$ 


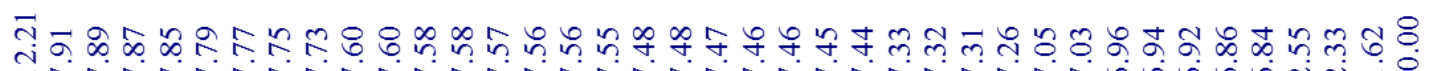

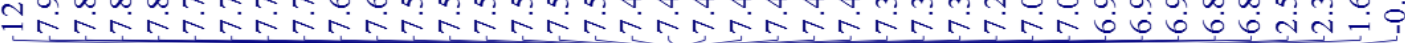
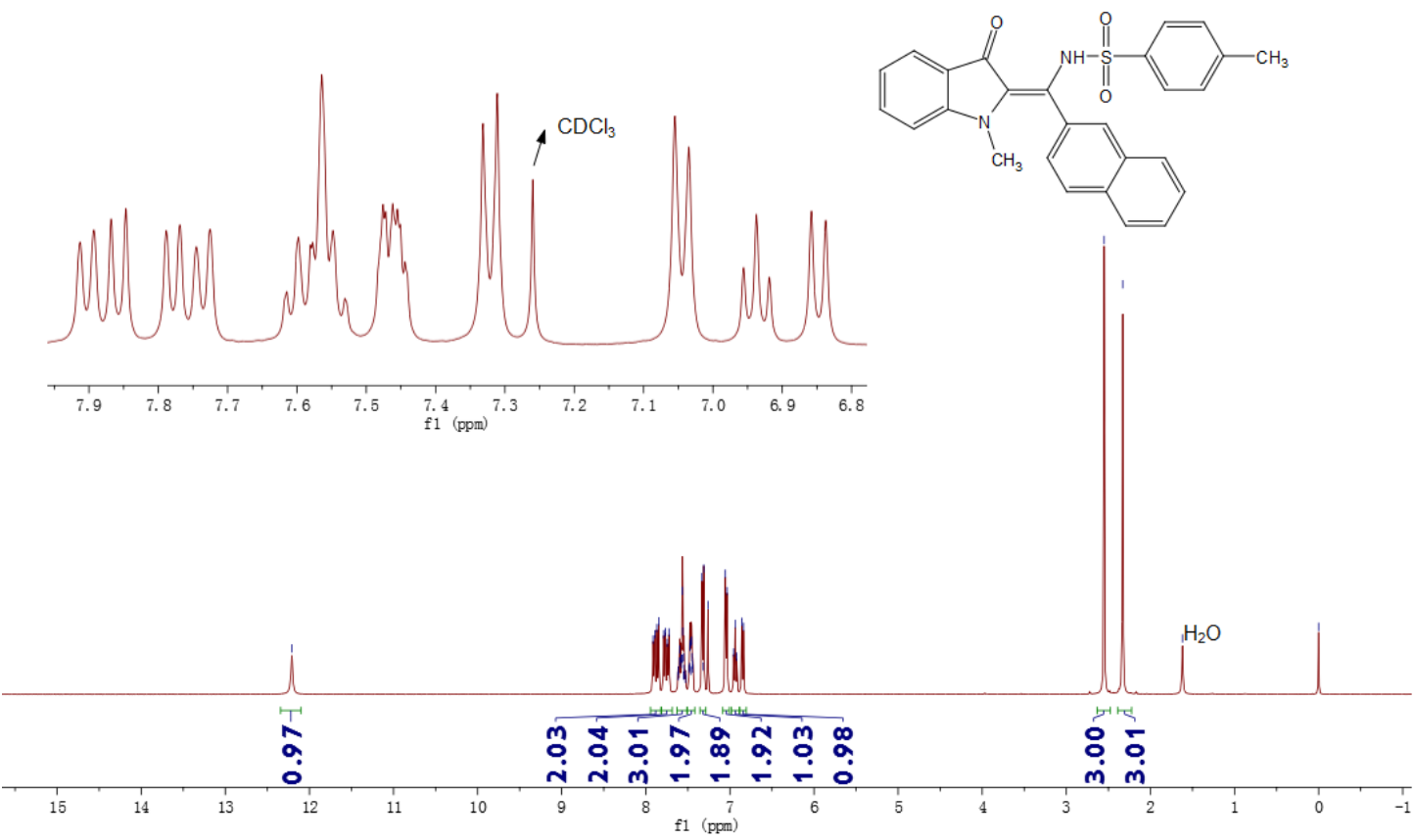

${ }^{1} \mathrm{H}$ NMR Spectrum for $3 \mathbf{m}\left(\mathrm{CDCl}_{3}, 400 \mathrm{MHz}\right)$

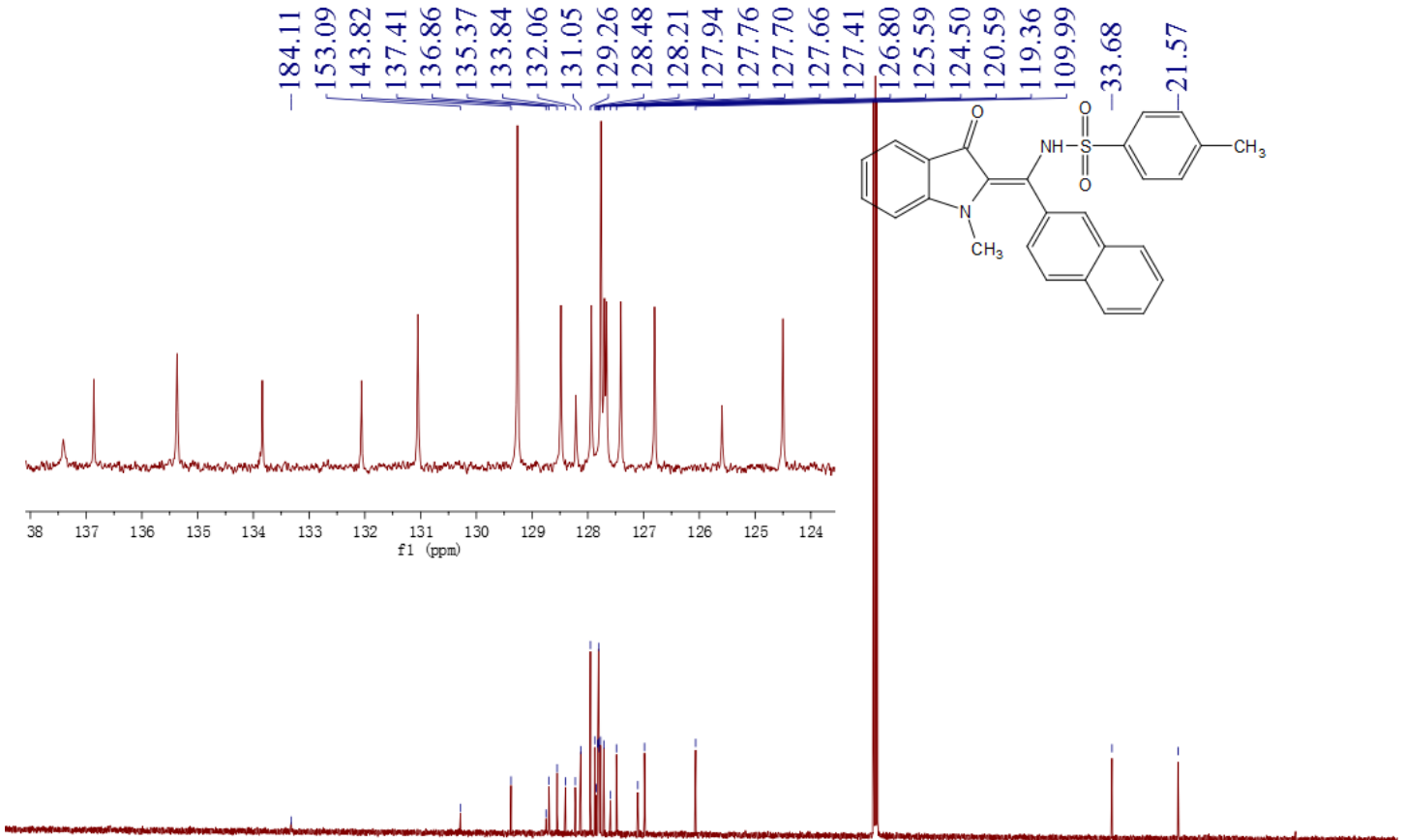

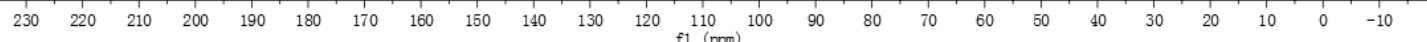

${ }^{13} \mathrm{CNMR}$ Spectrum for $3 \mathbf{m}\left(\mathrm{CDCl}_{3}, 100 \mathrm{MHz}\right)$ 


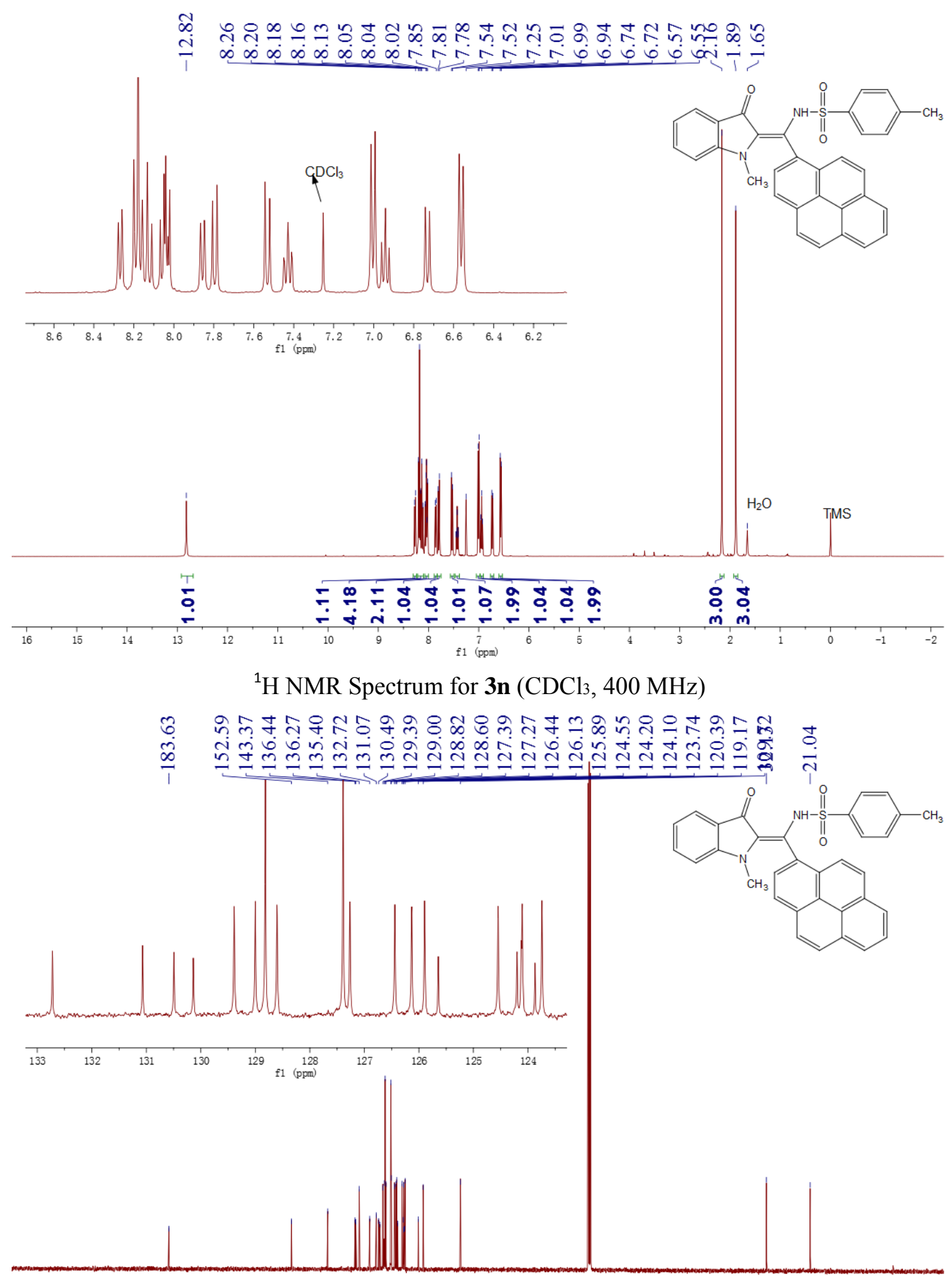

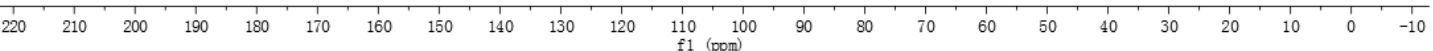

${ }^{13} \mathrm{CNMR}$ Spectrum for $3 \mathbf{n}\left(\mathrm{CDCl}_{3}, 100 \mathrm{MHz}\right)$ 


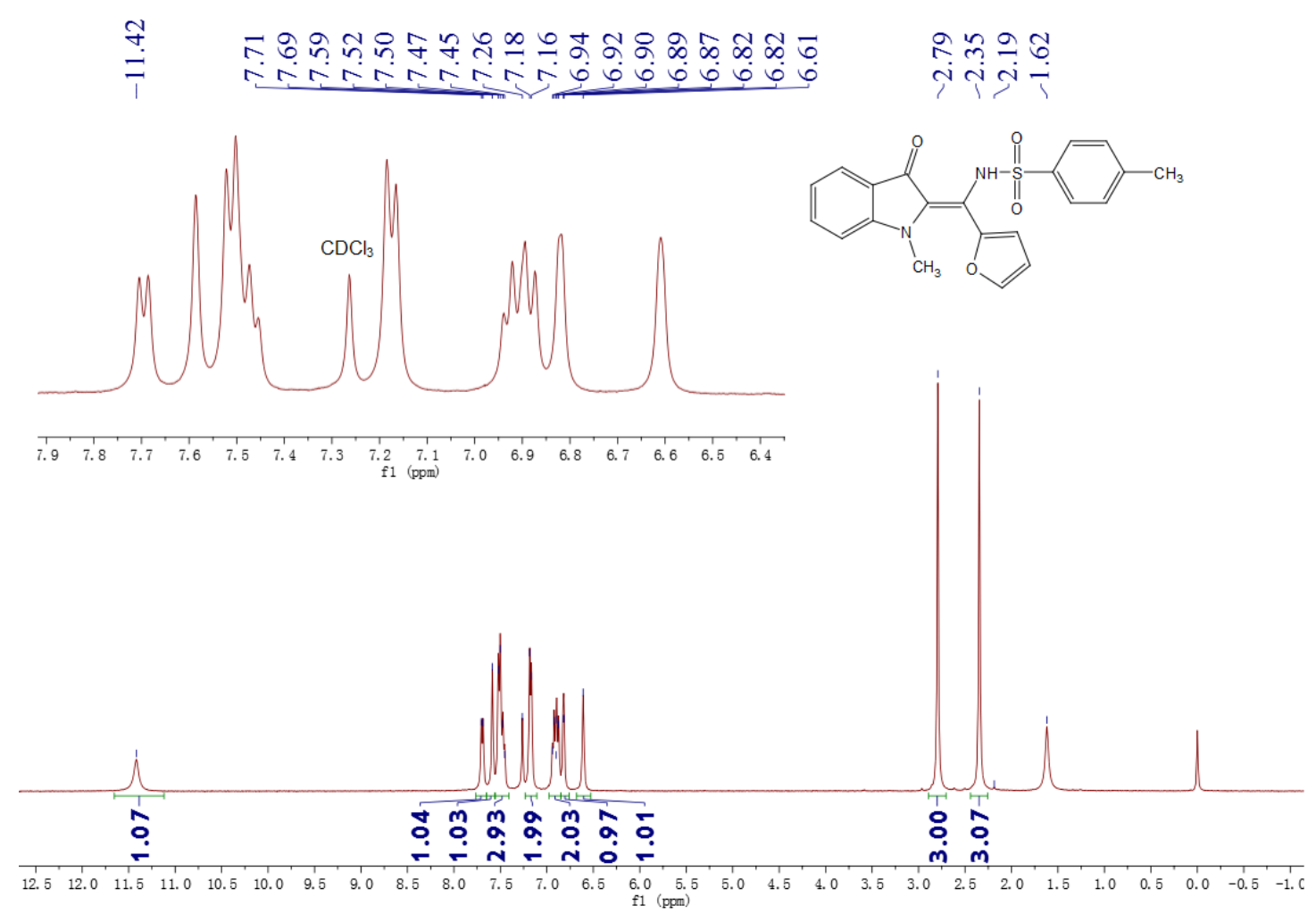

${ }^{1} \mathrm{H}$ NMR Spectrum for $30\left(\mathrm{CDCl}_{3}, 400 \mathrm{MHz}\right)$
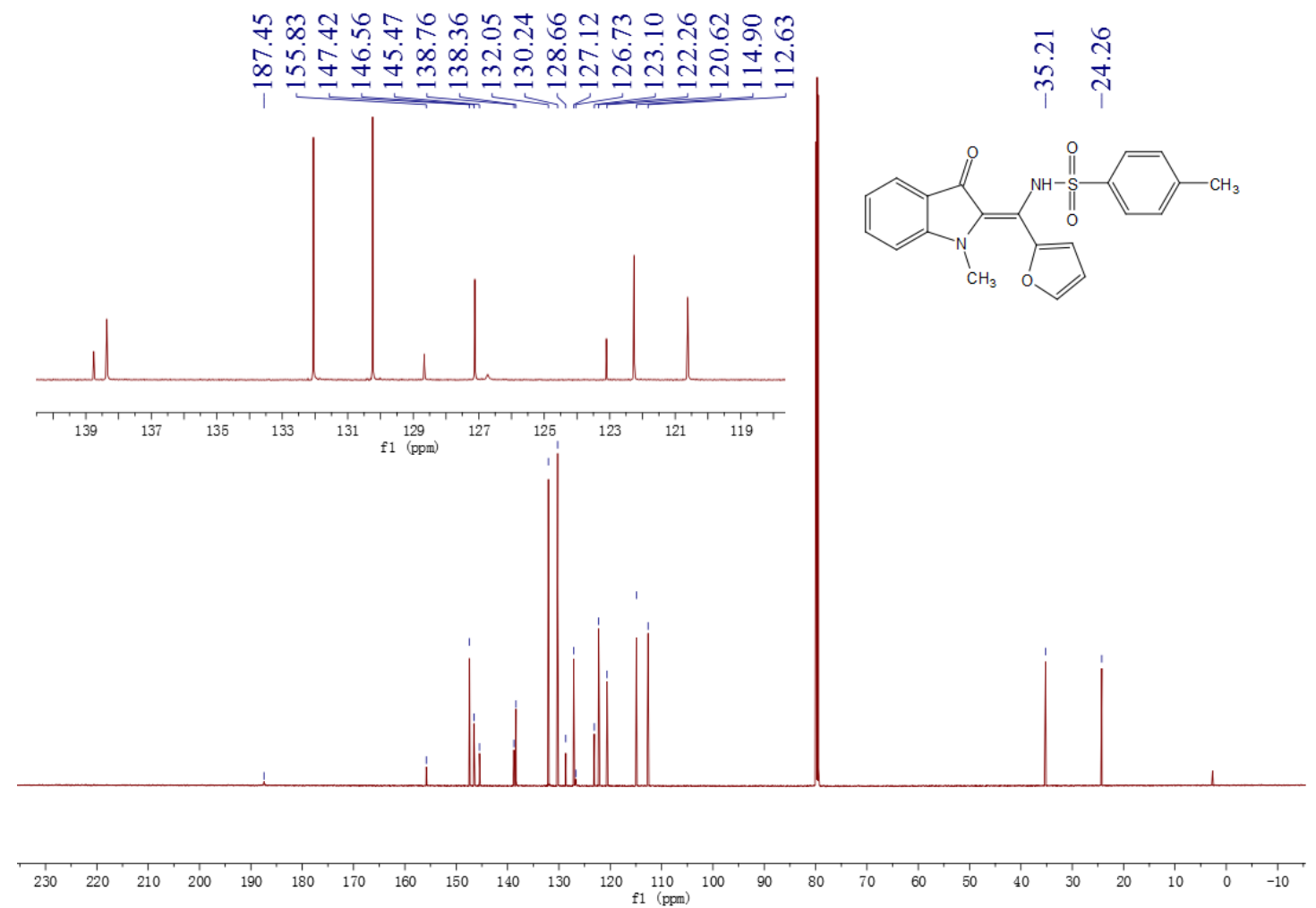

${ }^{13} \mathrm{CNMR}$ Spectrum for $3 \mathbf{0}\left(\mathrm{CDCl}_{3}, 150 \mathrm{MHz}\right)$ 


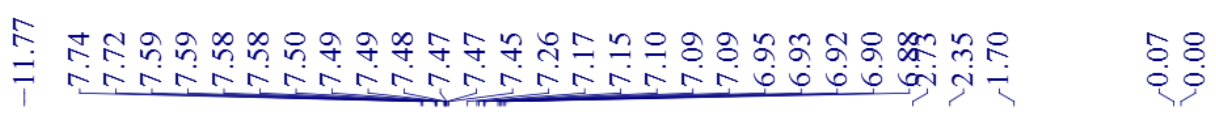

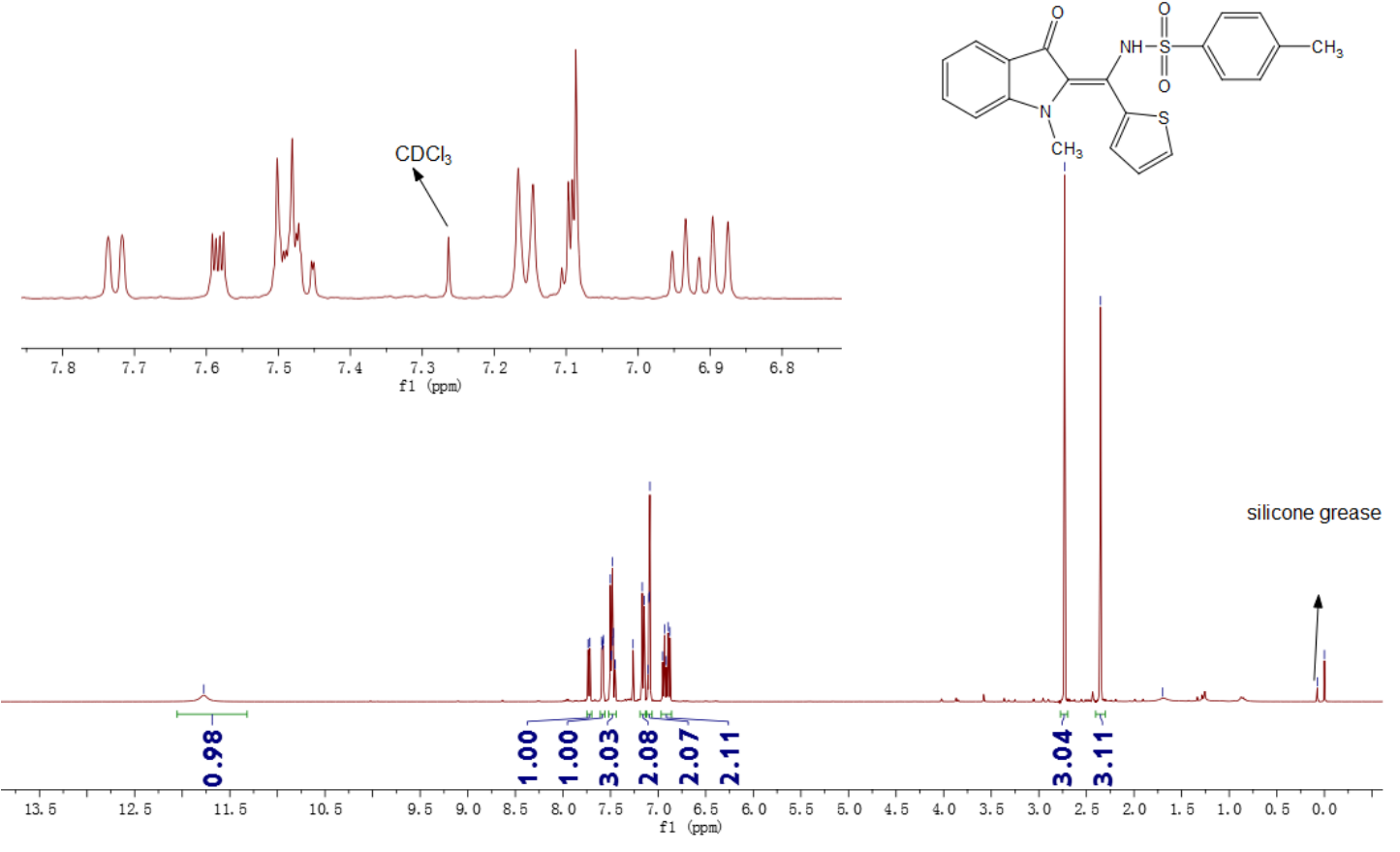

${ }^{1} \mathrm{H}$ NMR Spectrum for $3 \mathbf{p}\left(\mathrm{CDCl}_{3}, 400 \mathrm{MHz}\right)$

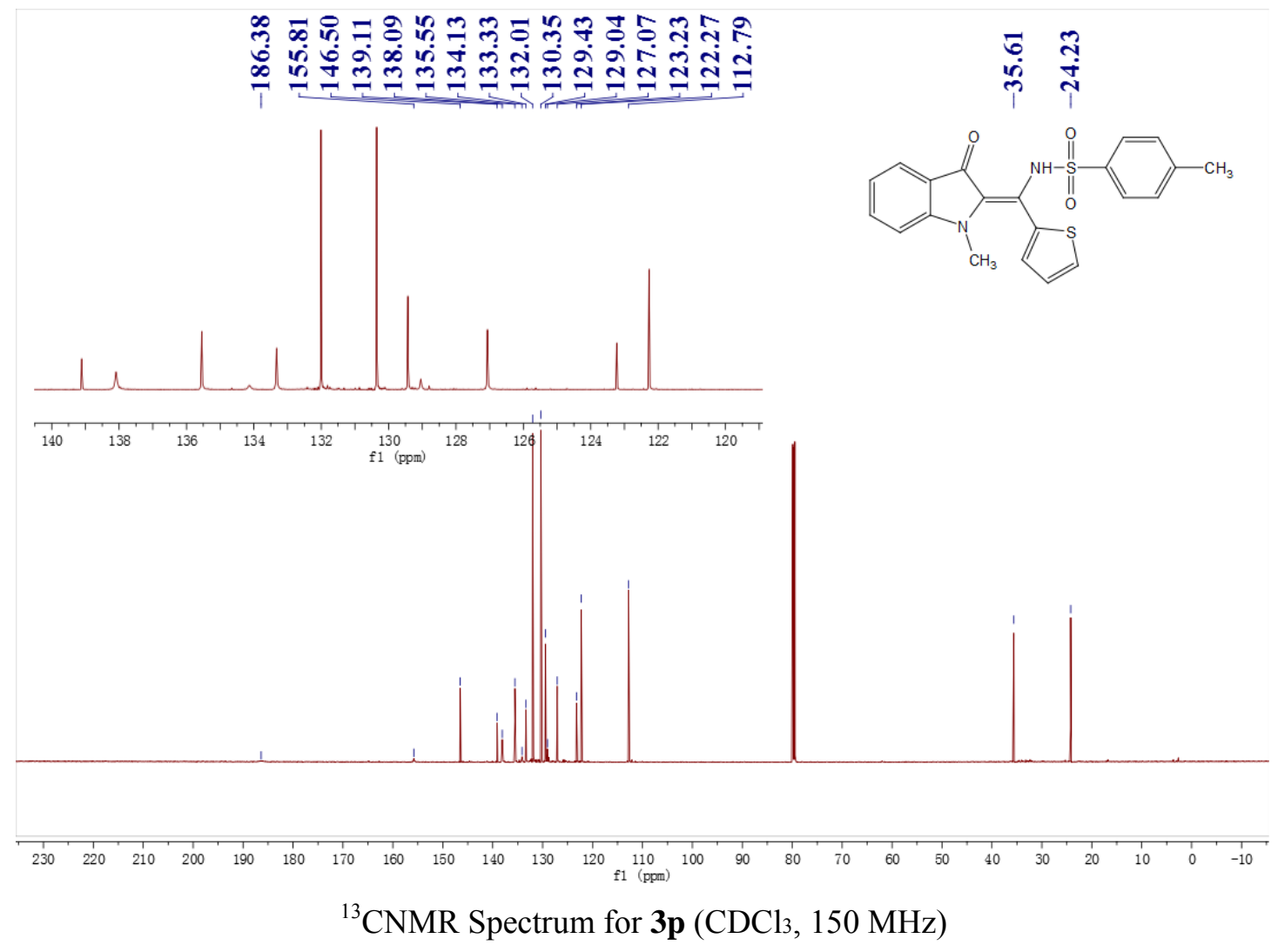




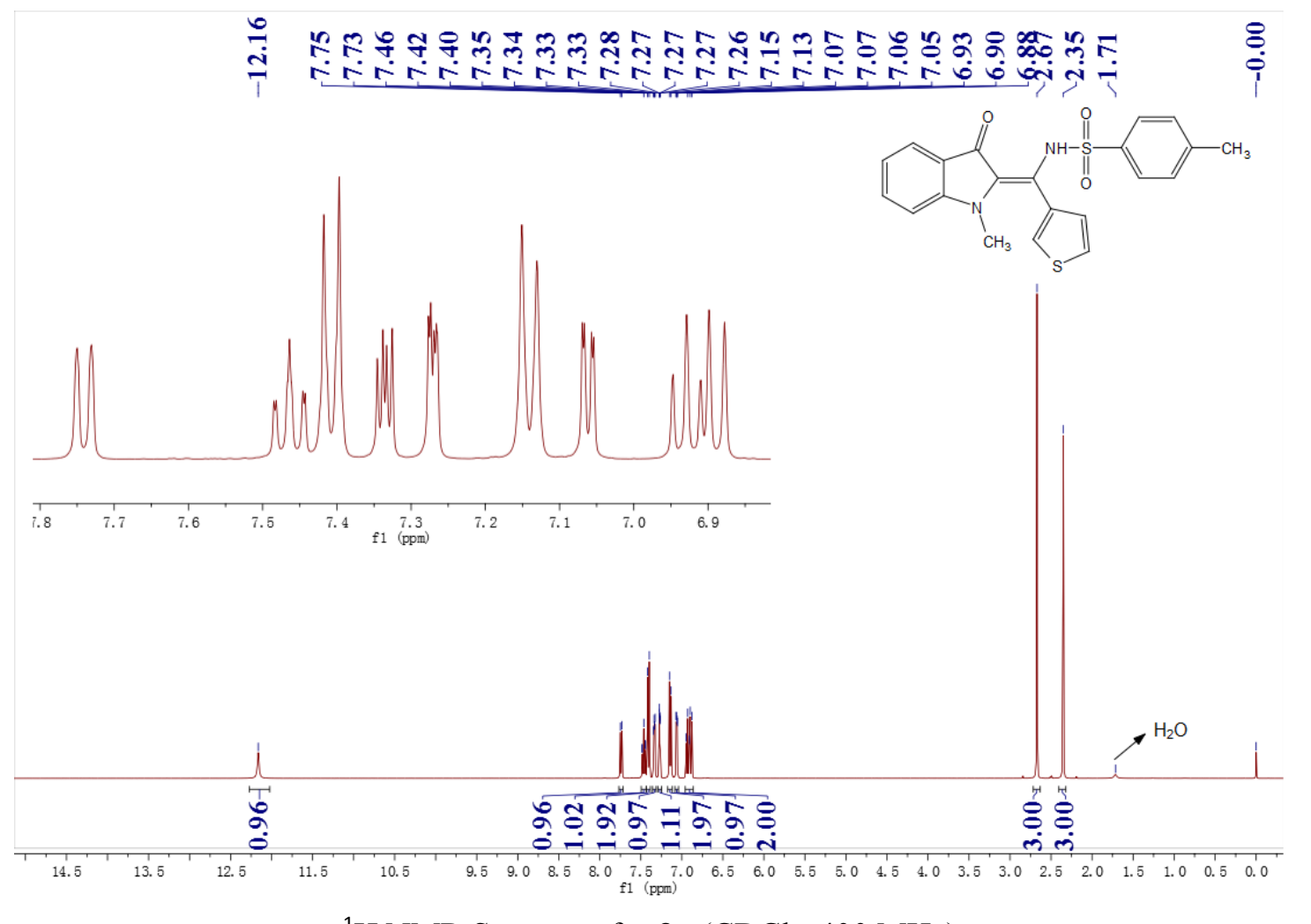

${ }^{1} \mathrm{H}$ NMR Spectrum for $\mathbf{3 q}\left(\mathrm{CDCl}_{3}, 400 \mathrm{MHz}\right)$

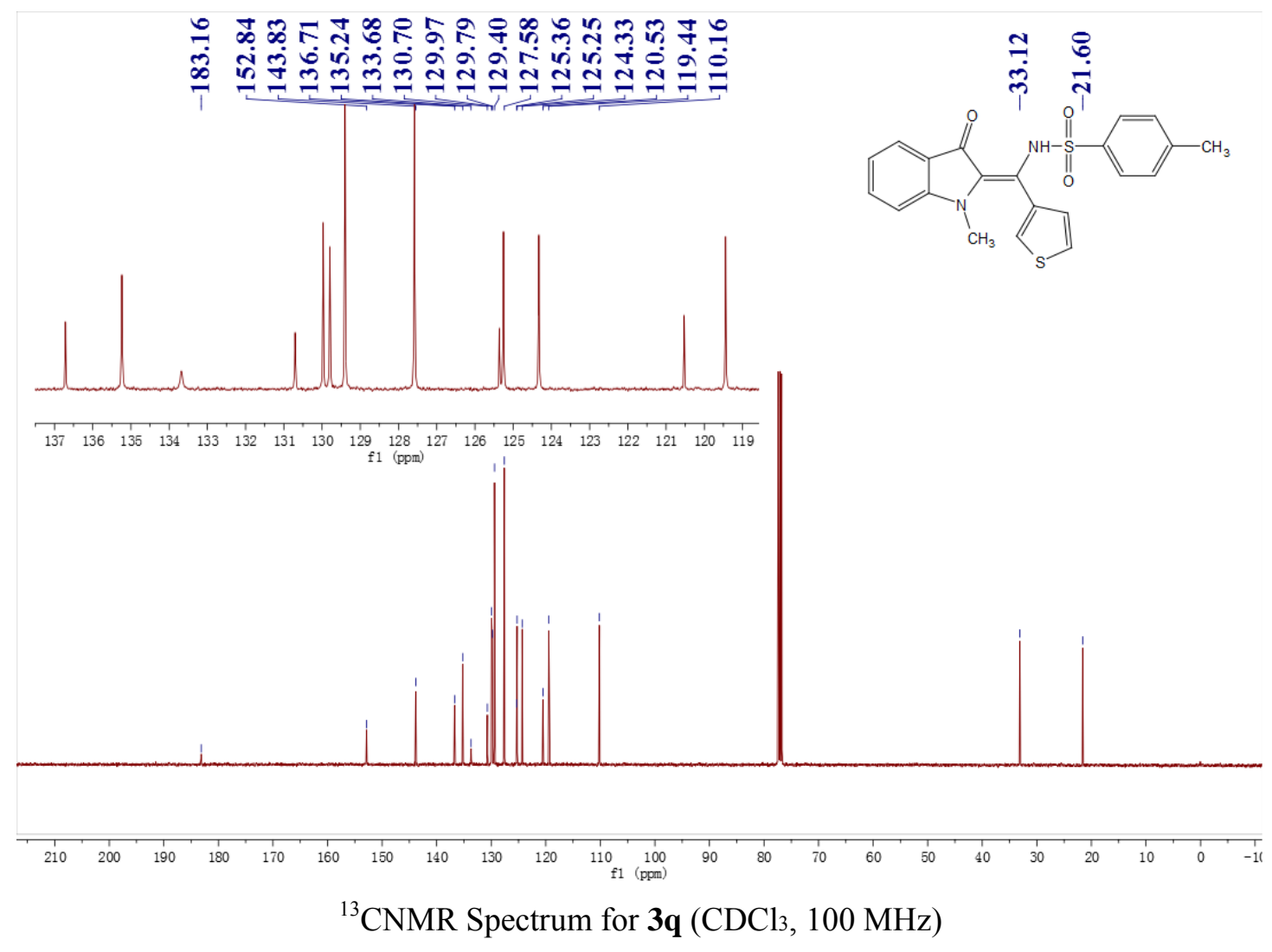




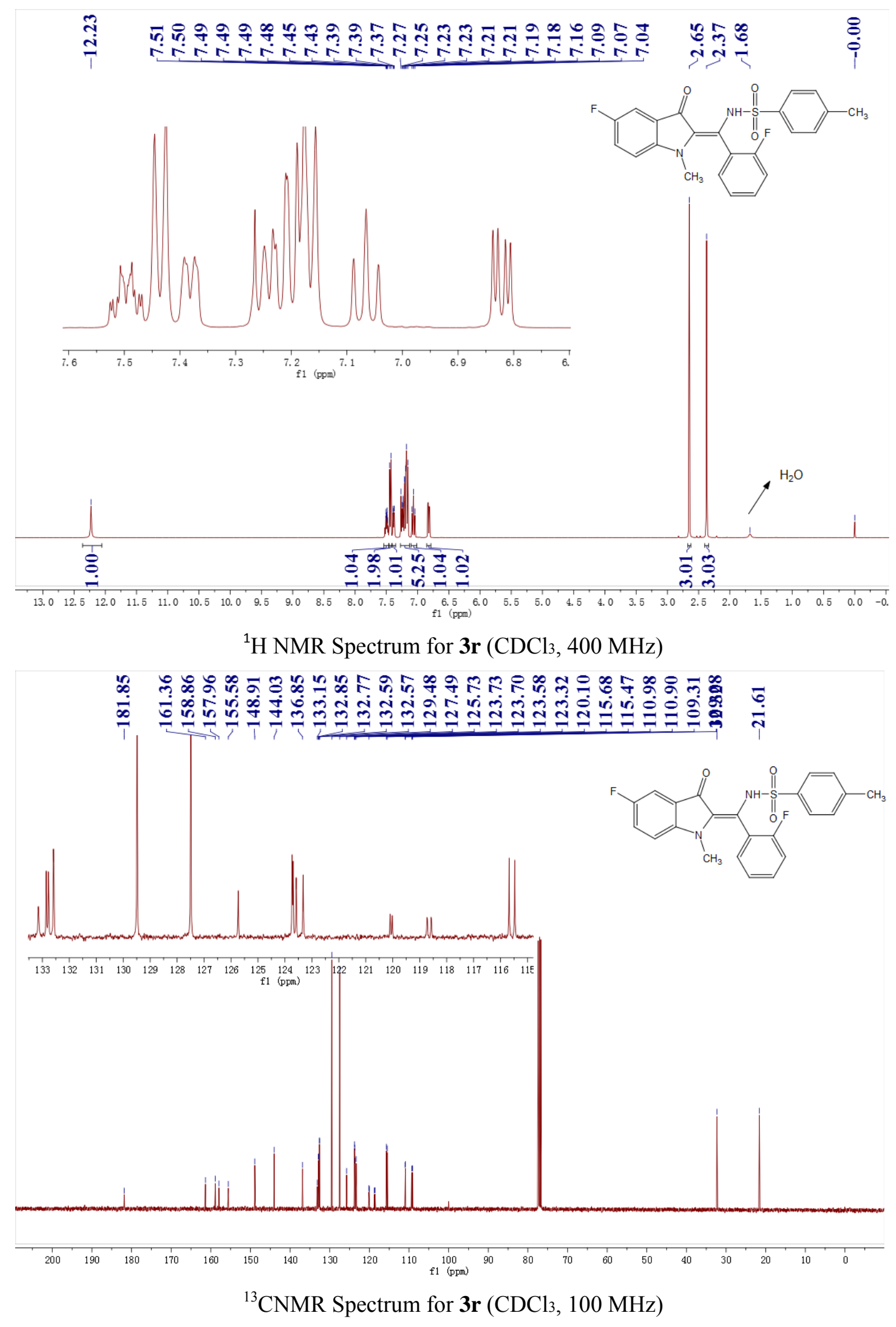



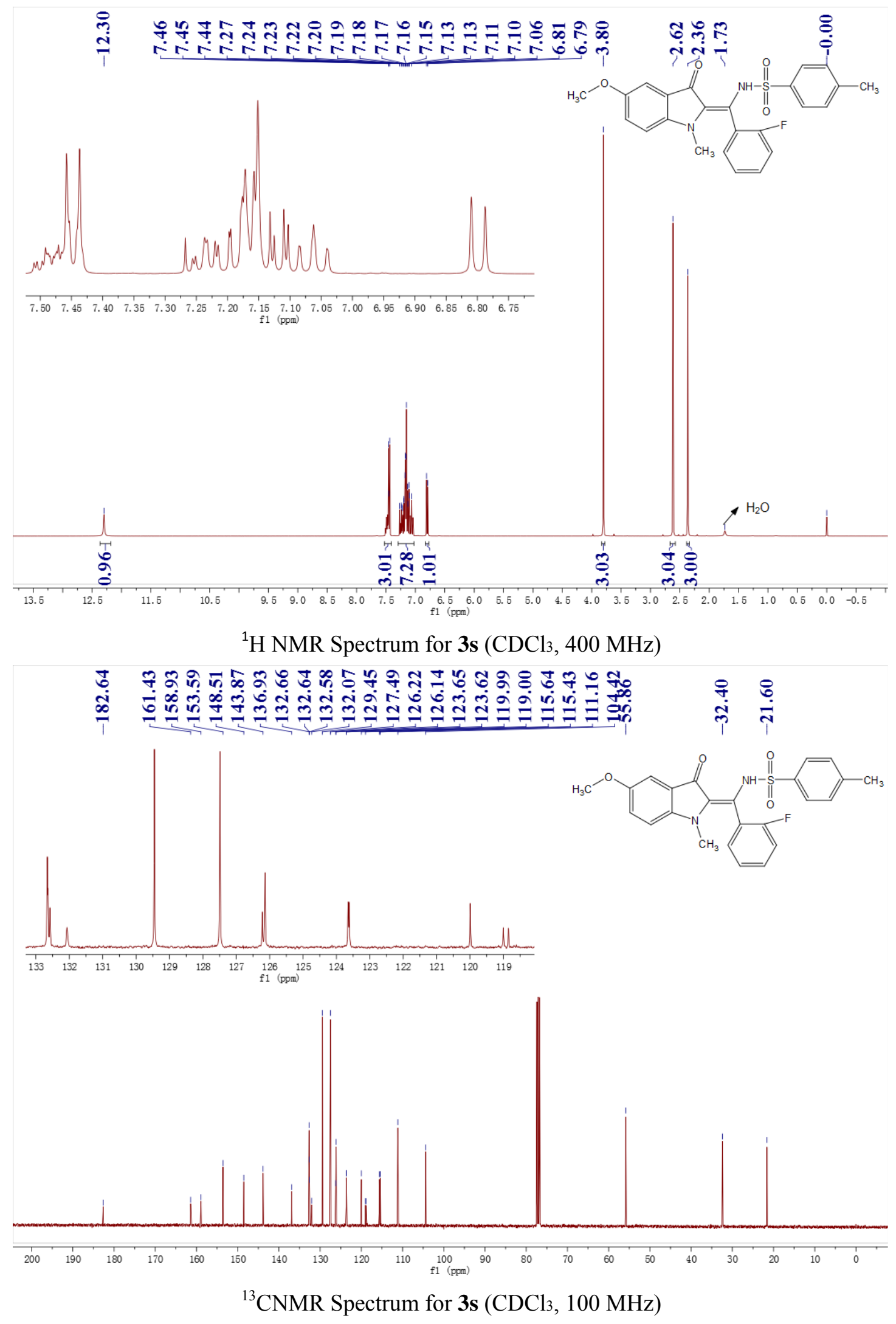


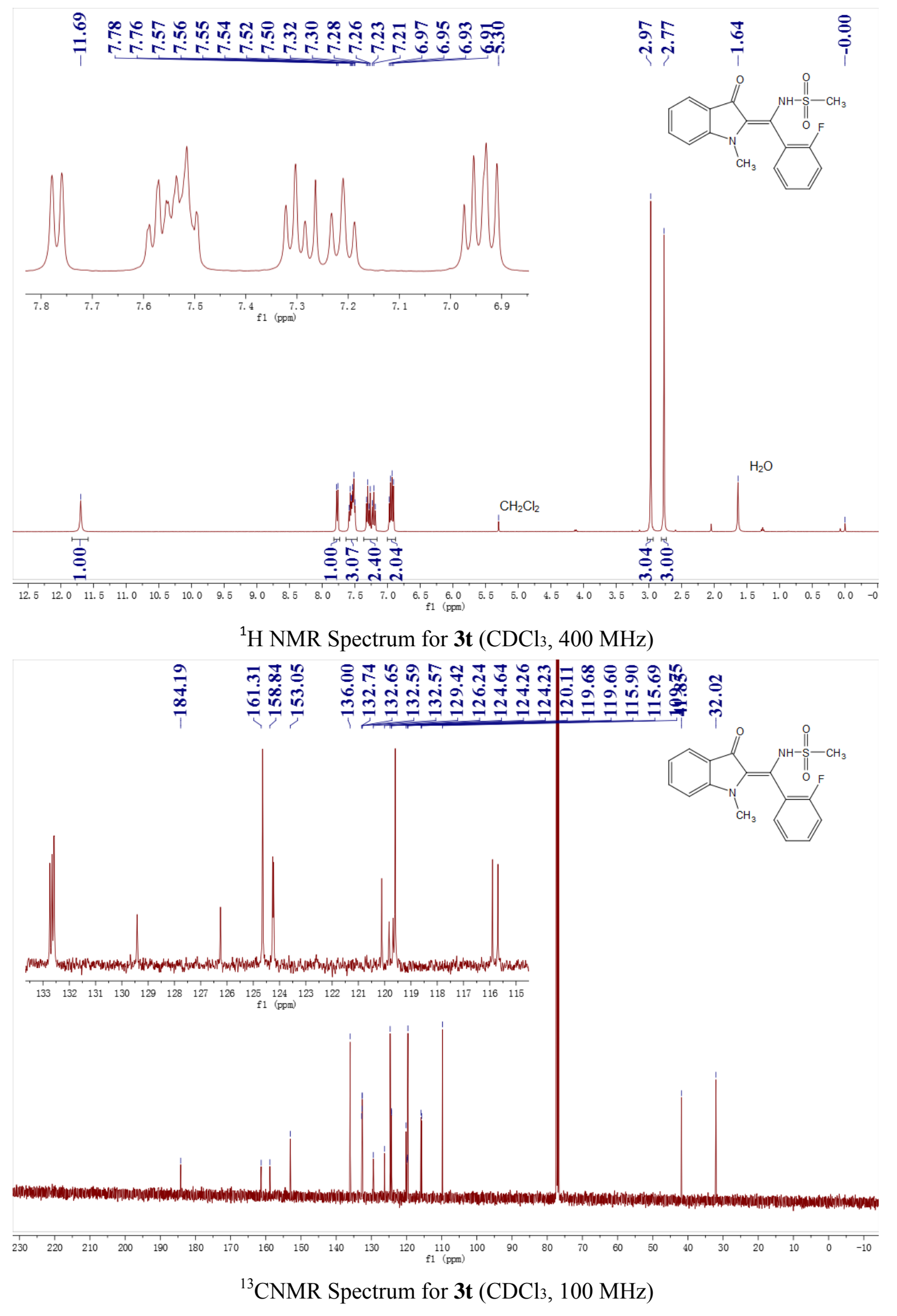




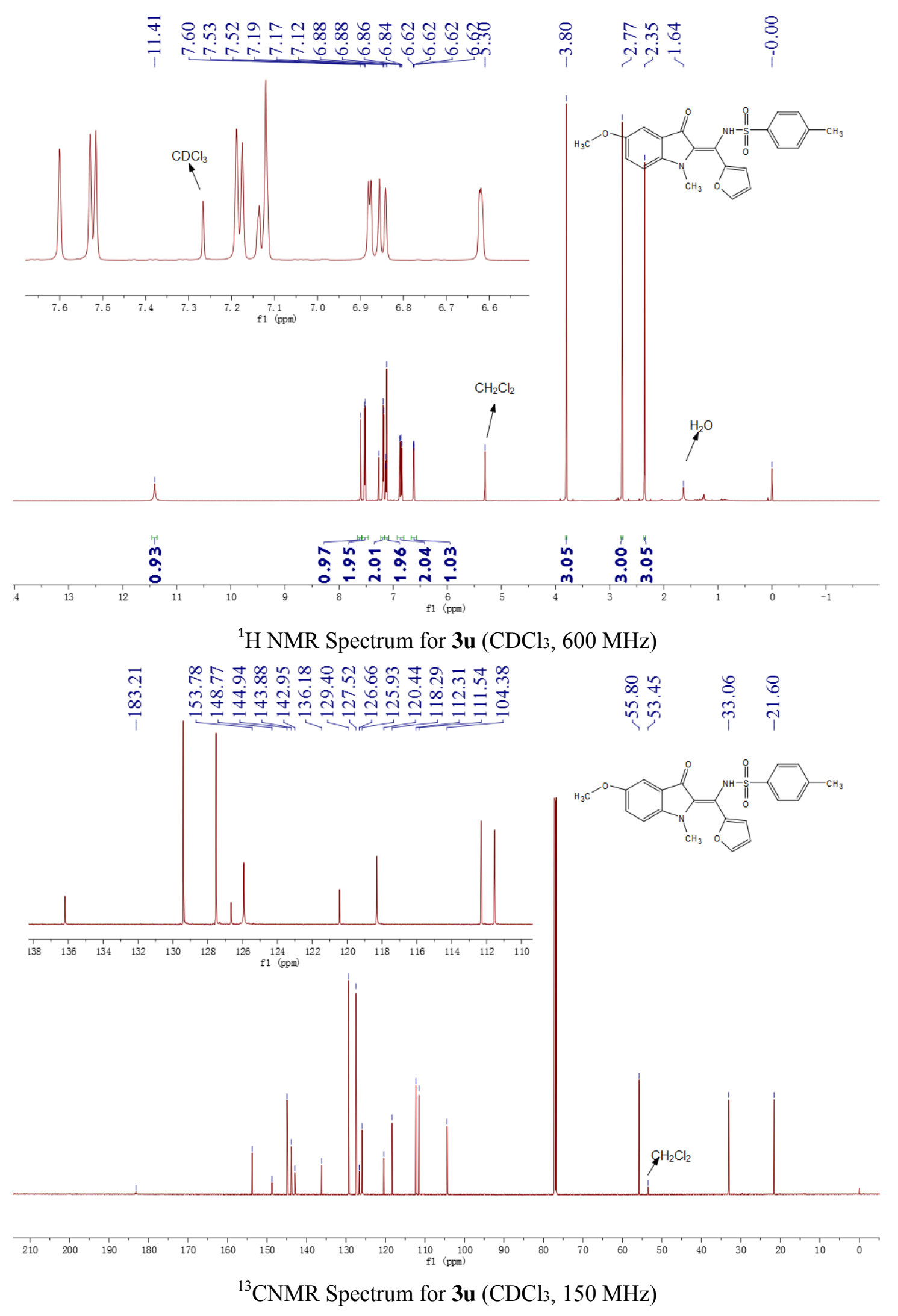




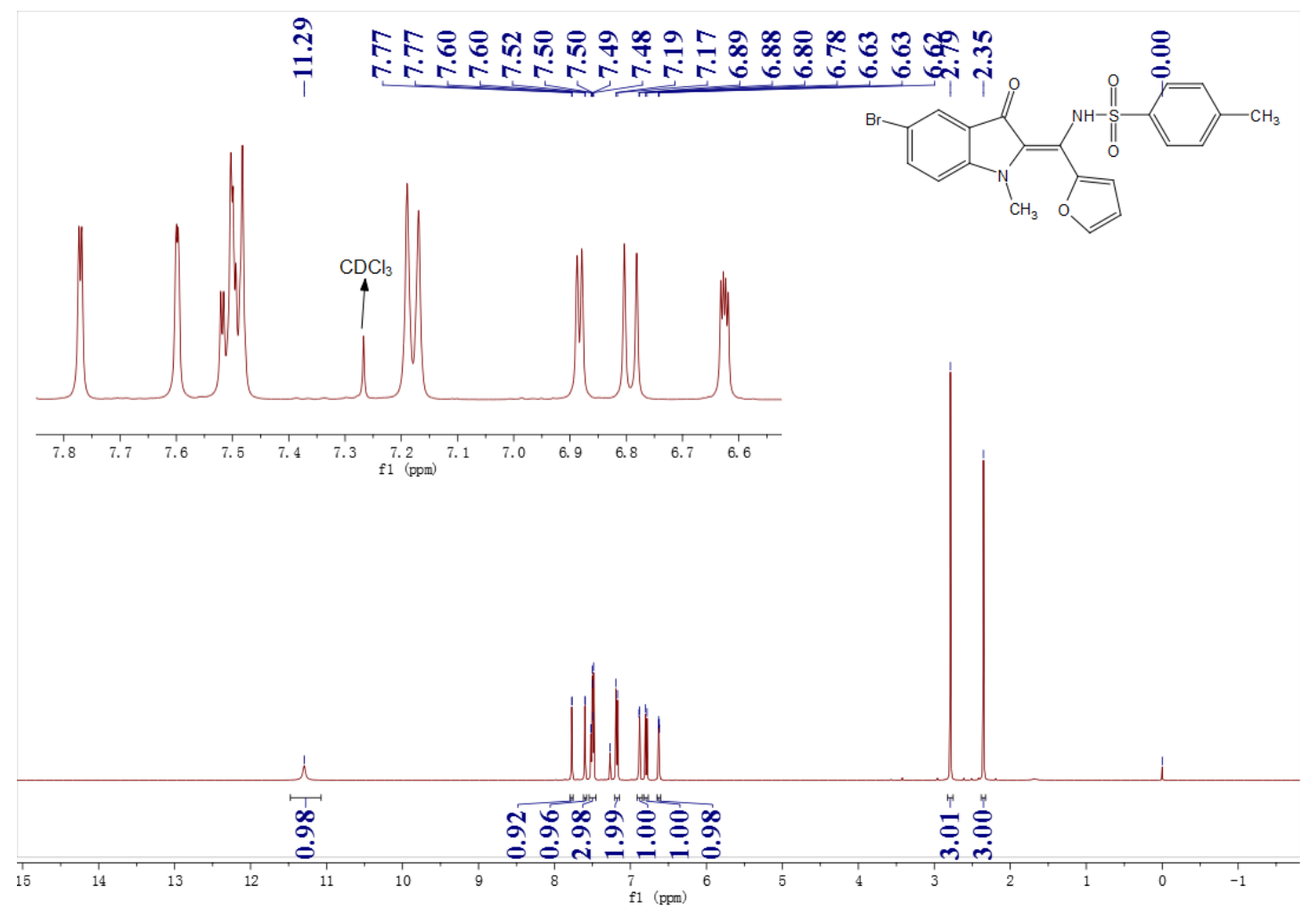

${ }^{1} \mathrm{H}$ NMR Spectrum for $3 \mathbf{v}\left(\mathrm{CDCl}_{3}, 400 \mathrm{MHz}\right)$

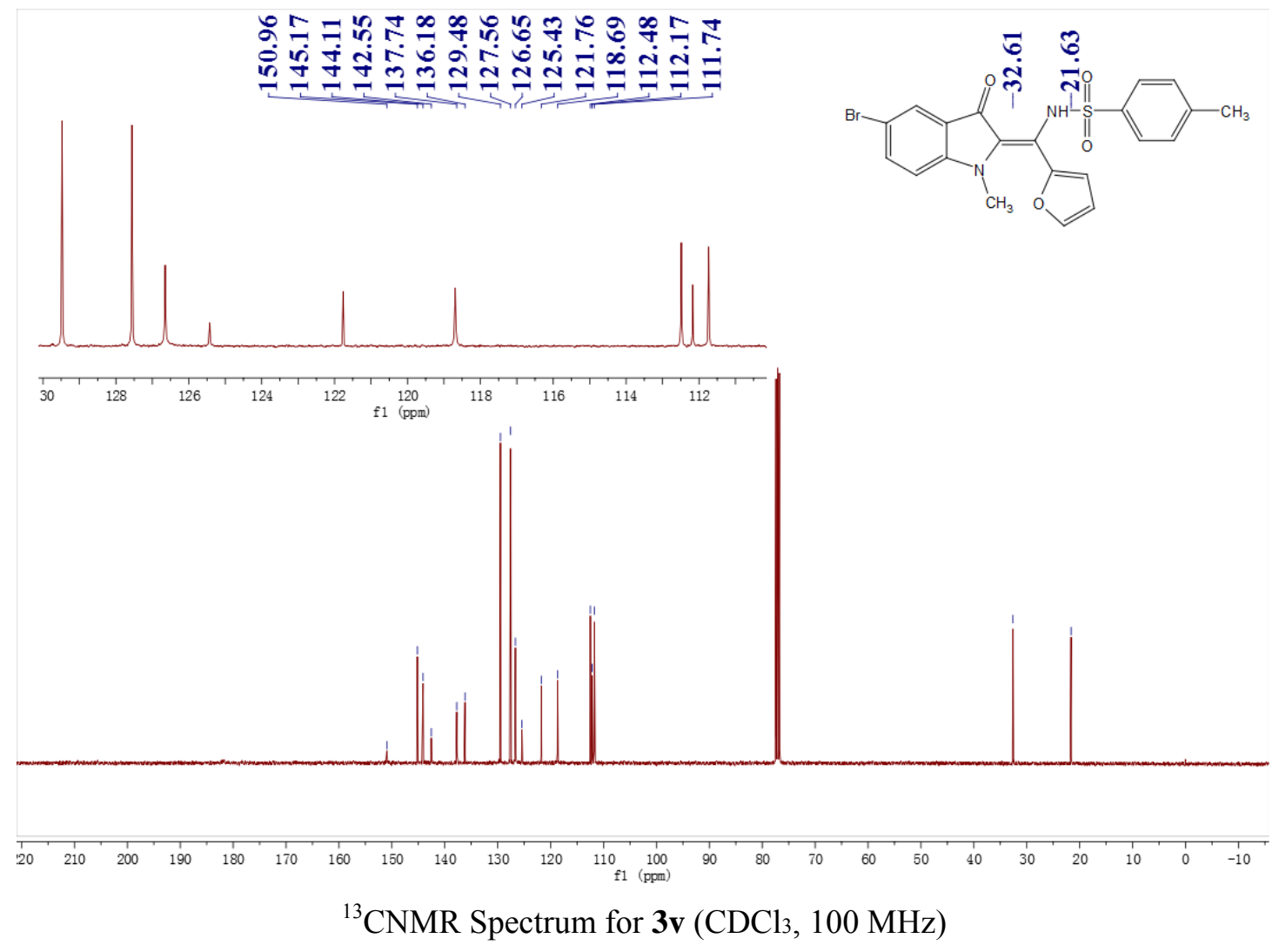




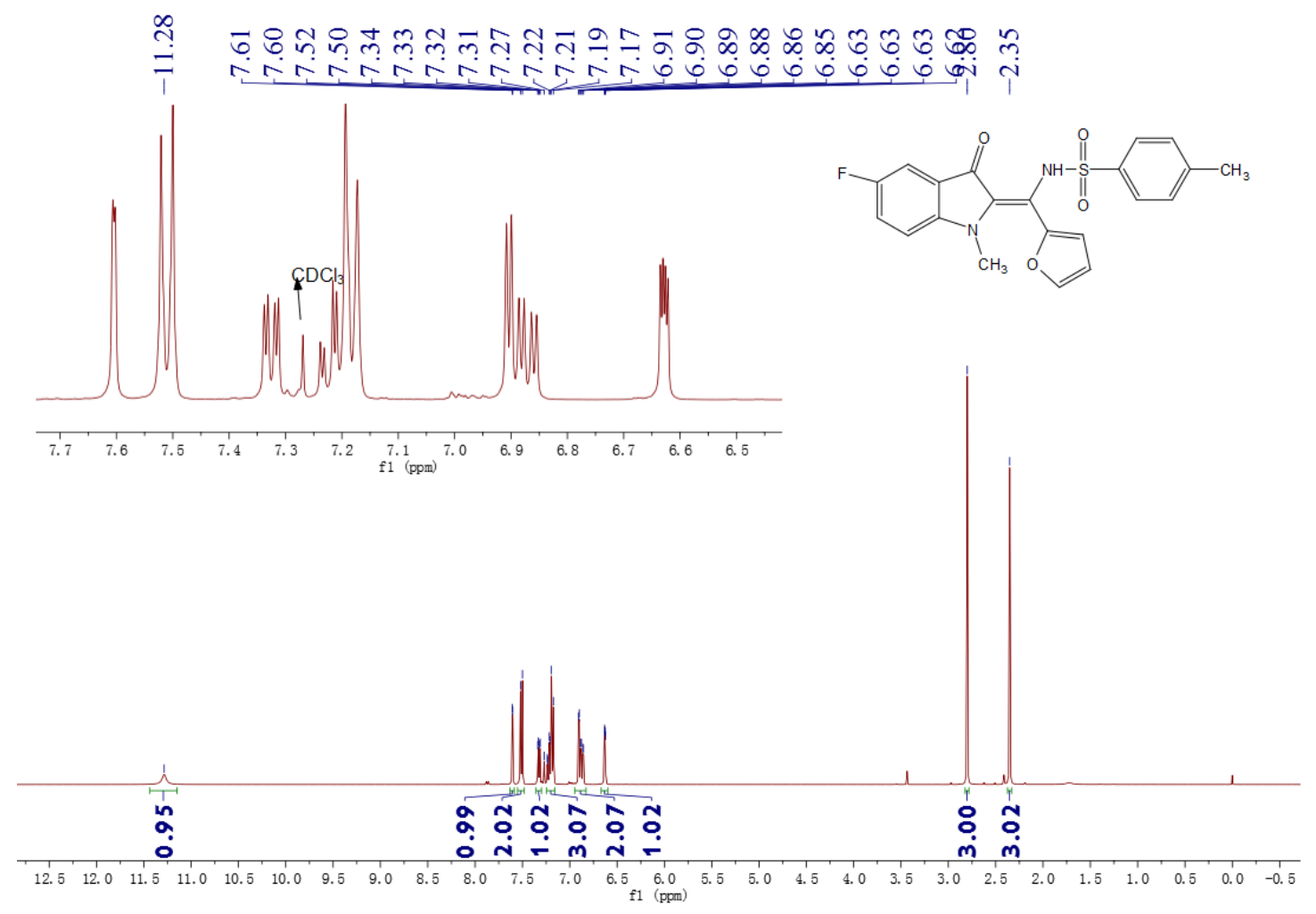

${ }^{1} \mathrm{H}$ NMR Spectrum for $3 \mathrm{w}\left(\mathrm{CDCl}_{3}, 400 \mathrm{MHz}\right)$

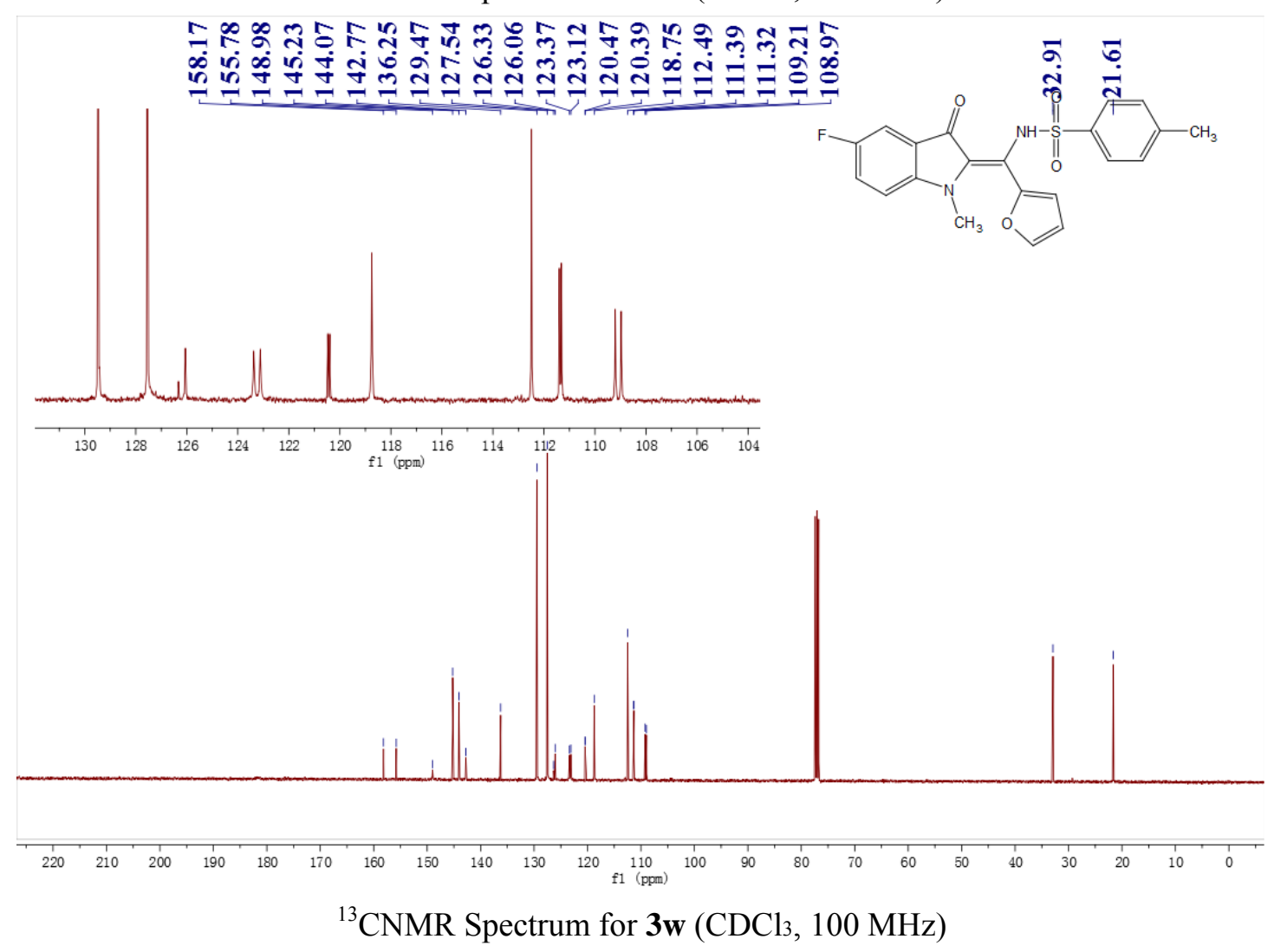




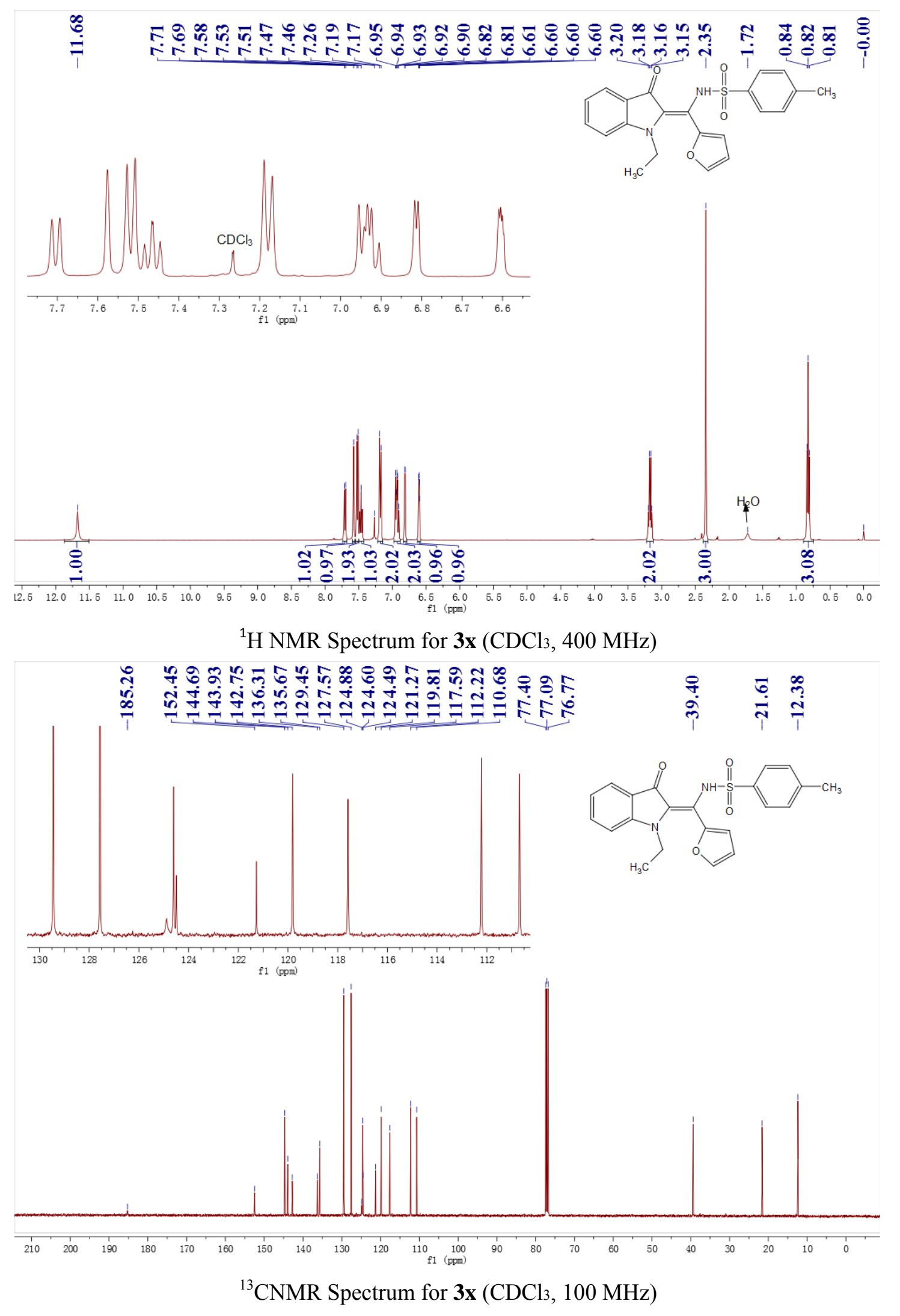




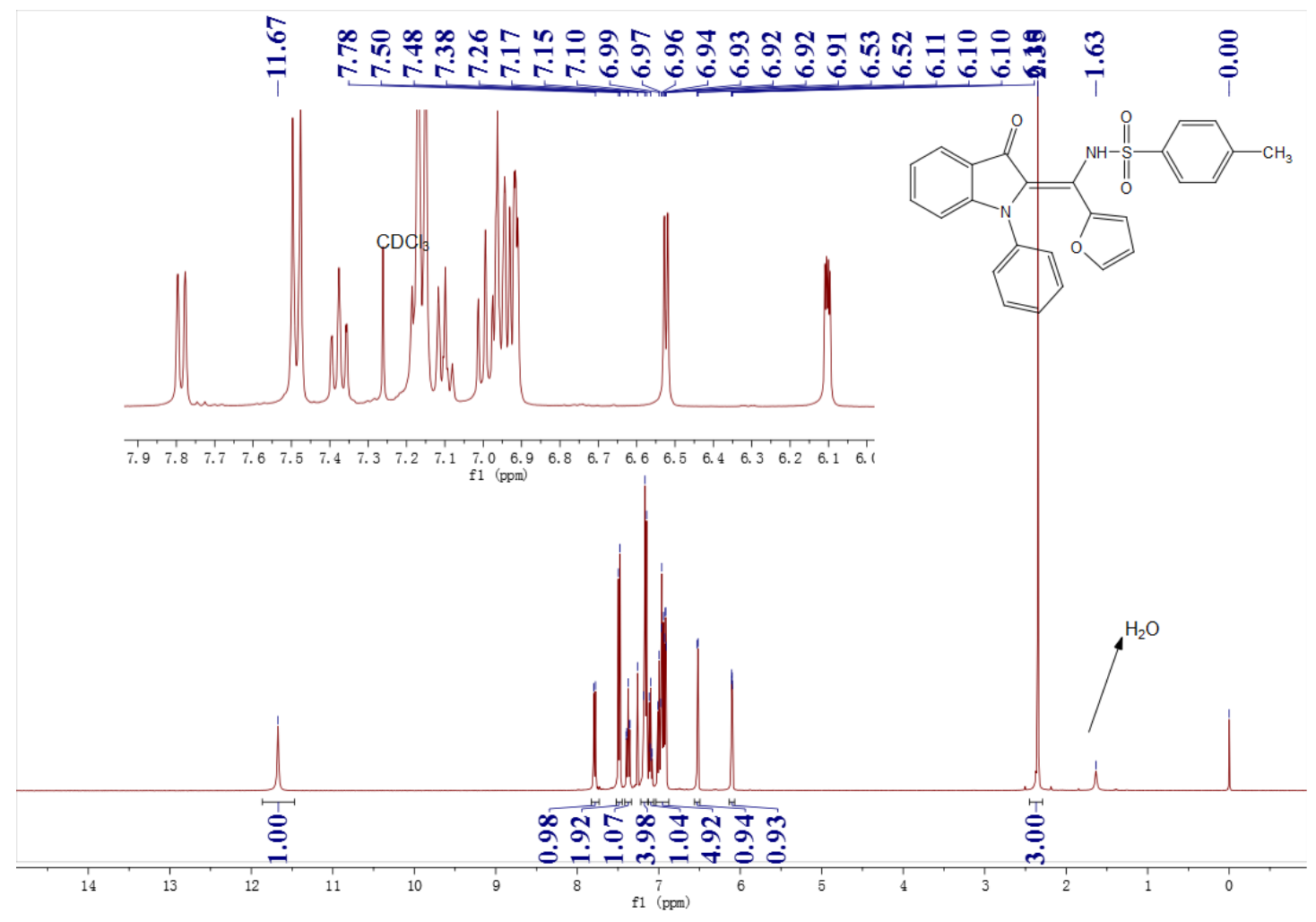

${ }^{1} \mathrm{H}$ NMR Spectrum for $\mathbf{3 y}\left(\mathrm{CDCl}_{3}, 400 \mathrm{MHz}\right)$

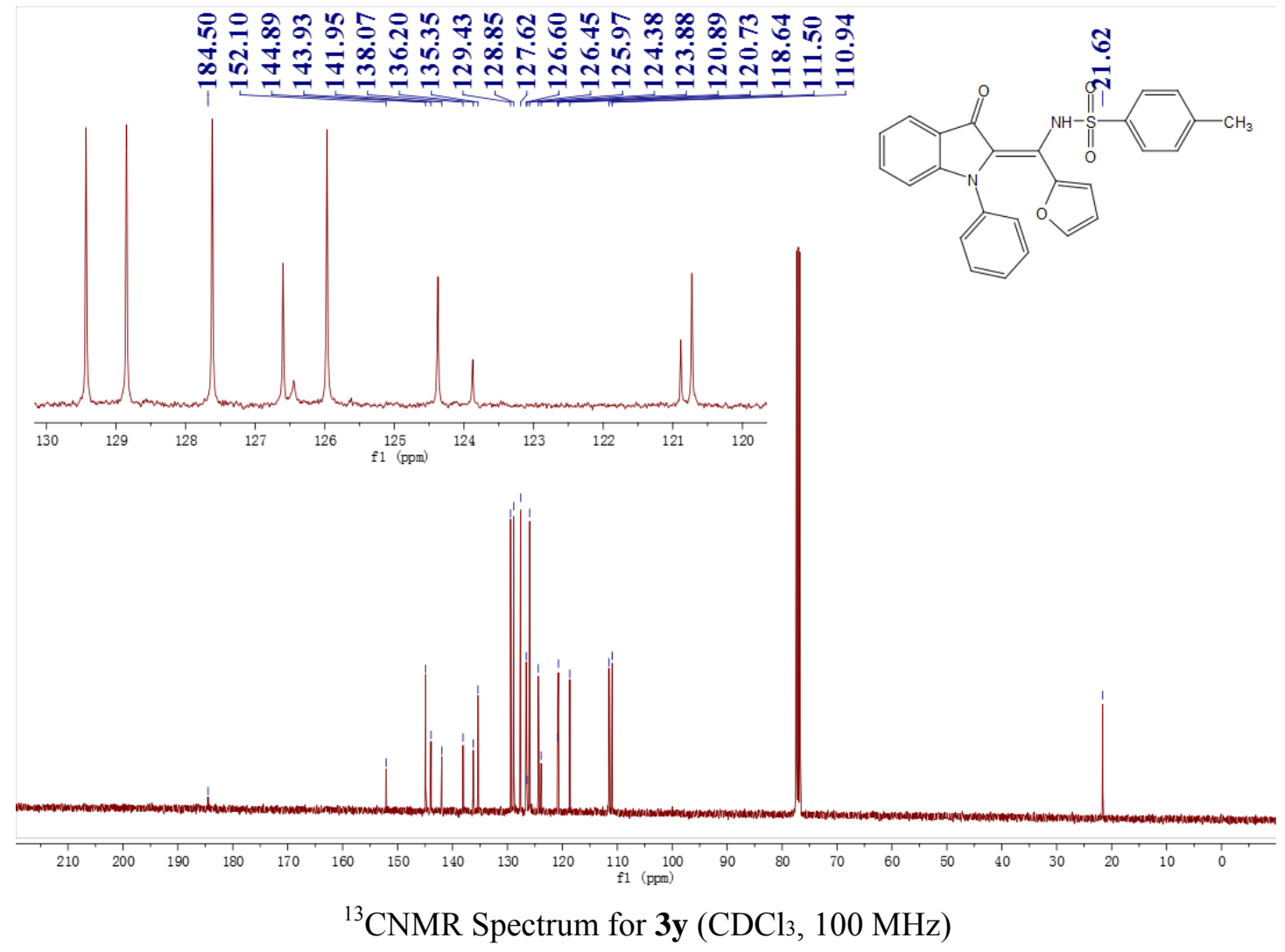




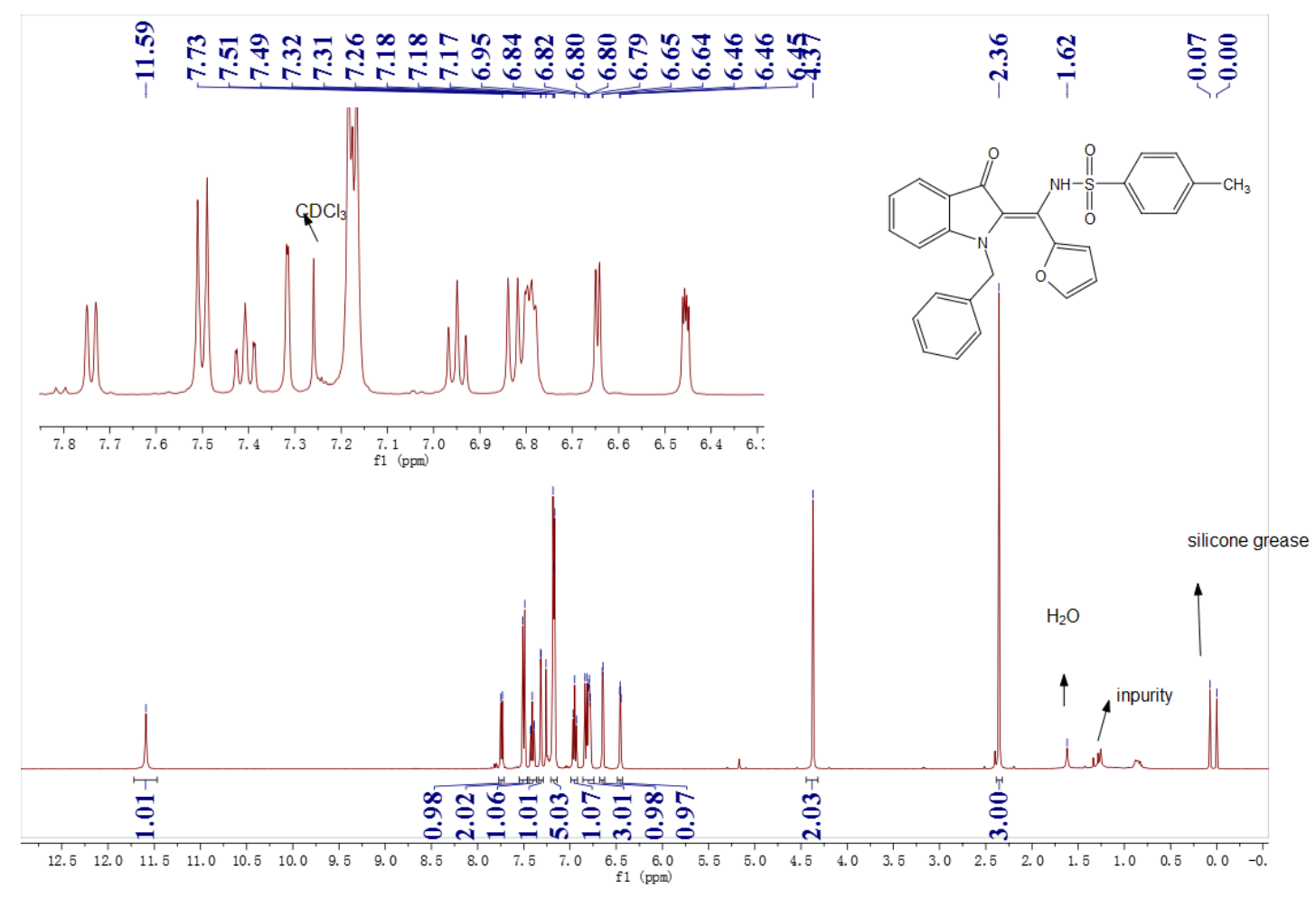

${ }^{1} \mathrm{H}$ NMR Spectrum for $\mathbf{3 z}\left(\mathrm{CDCl}_{3}, 400 \mathrm{MHz}\right)$

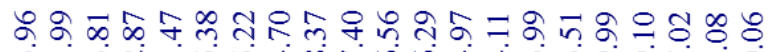

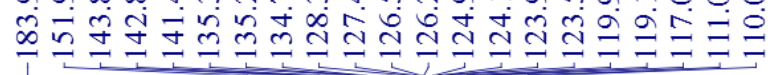

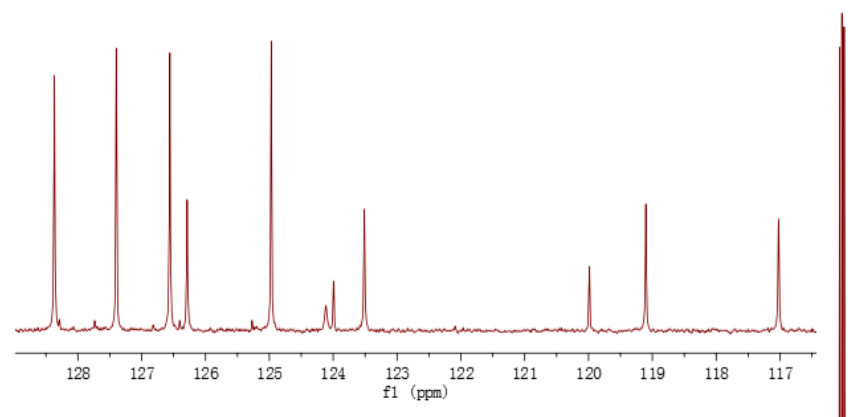

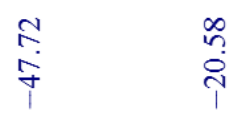

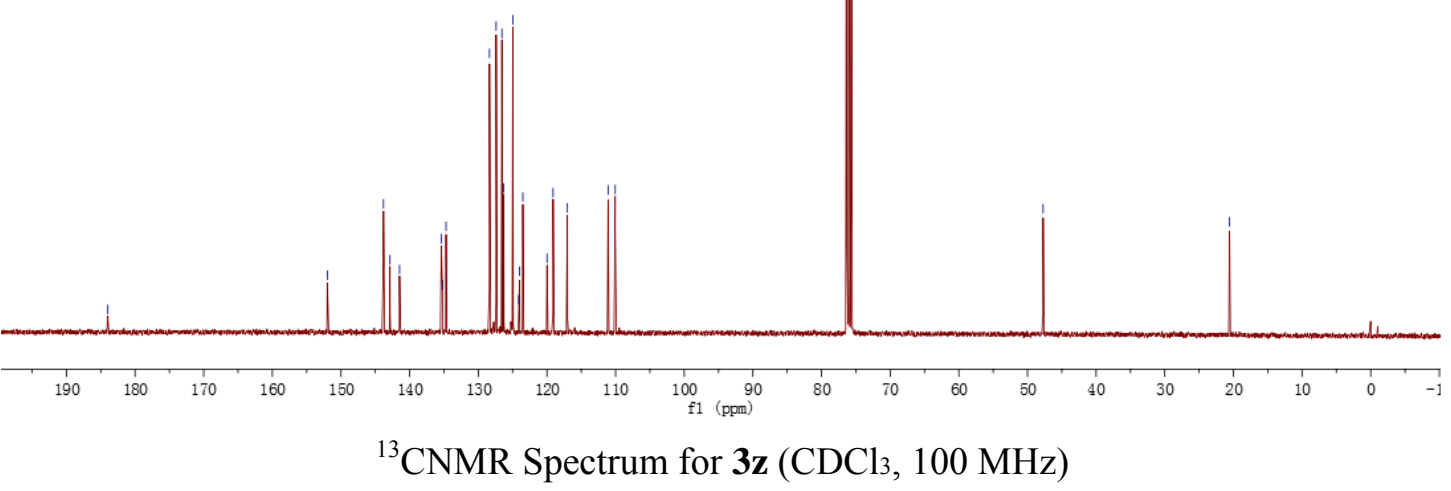




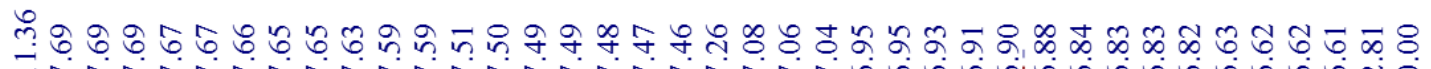

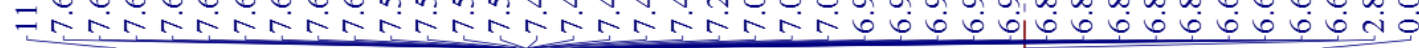
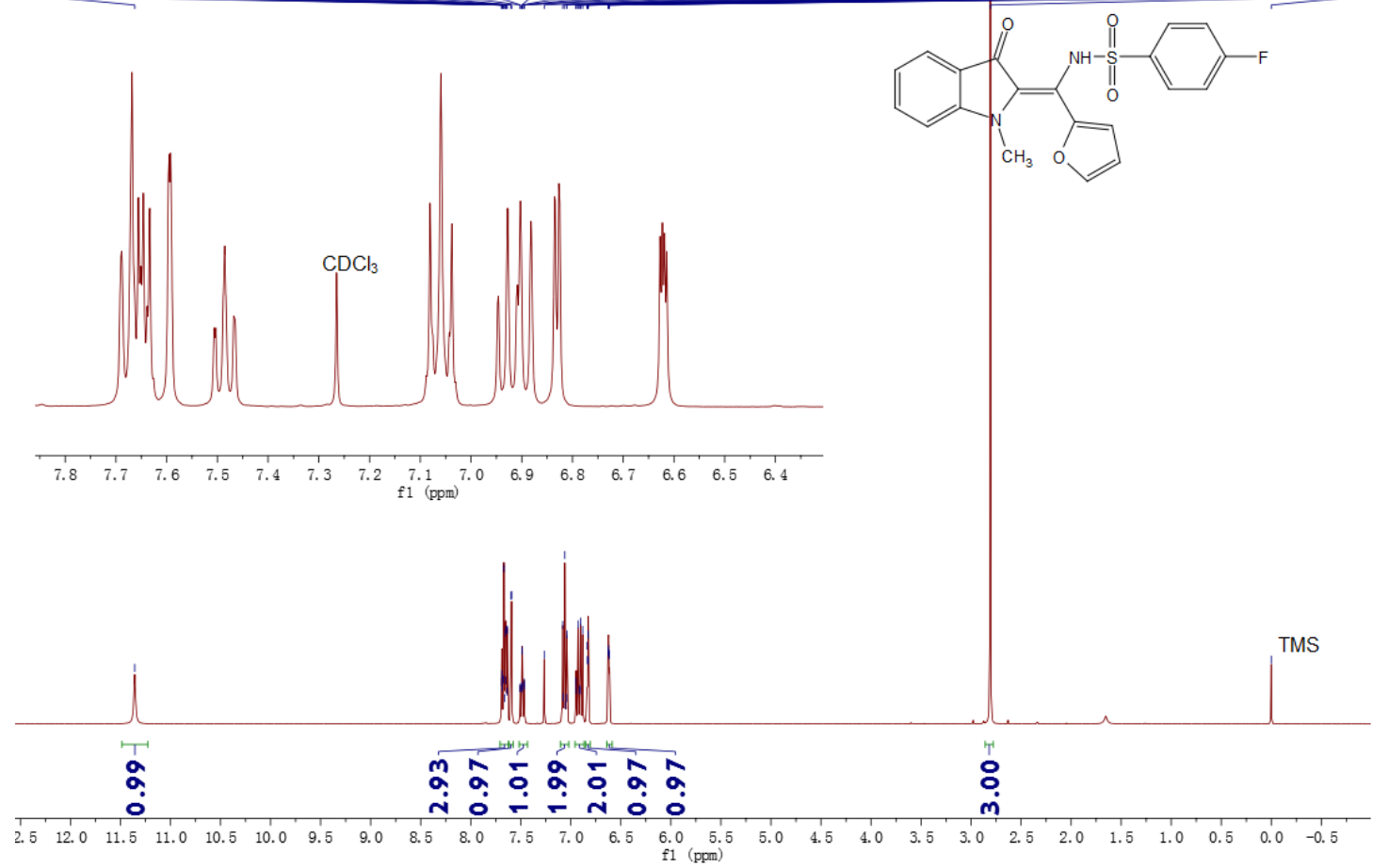

${ }^{1} \mathrm{H}$ NMR Spectrum for $\mathbf{3 A}\left(\mathrm{CDCl}_{3}, 400 \mathrm{MHz}\right)$

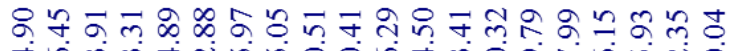

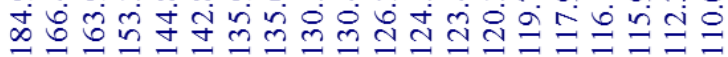
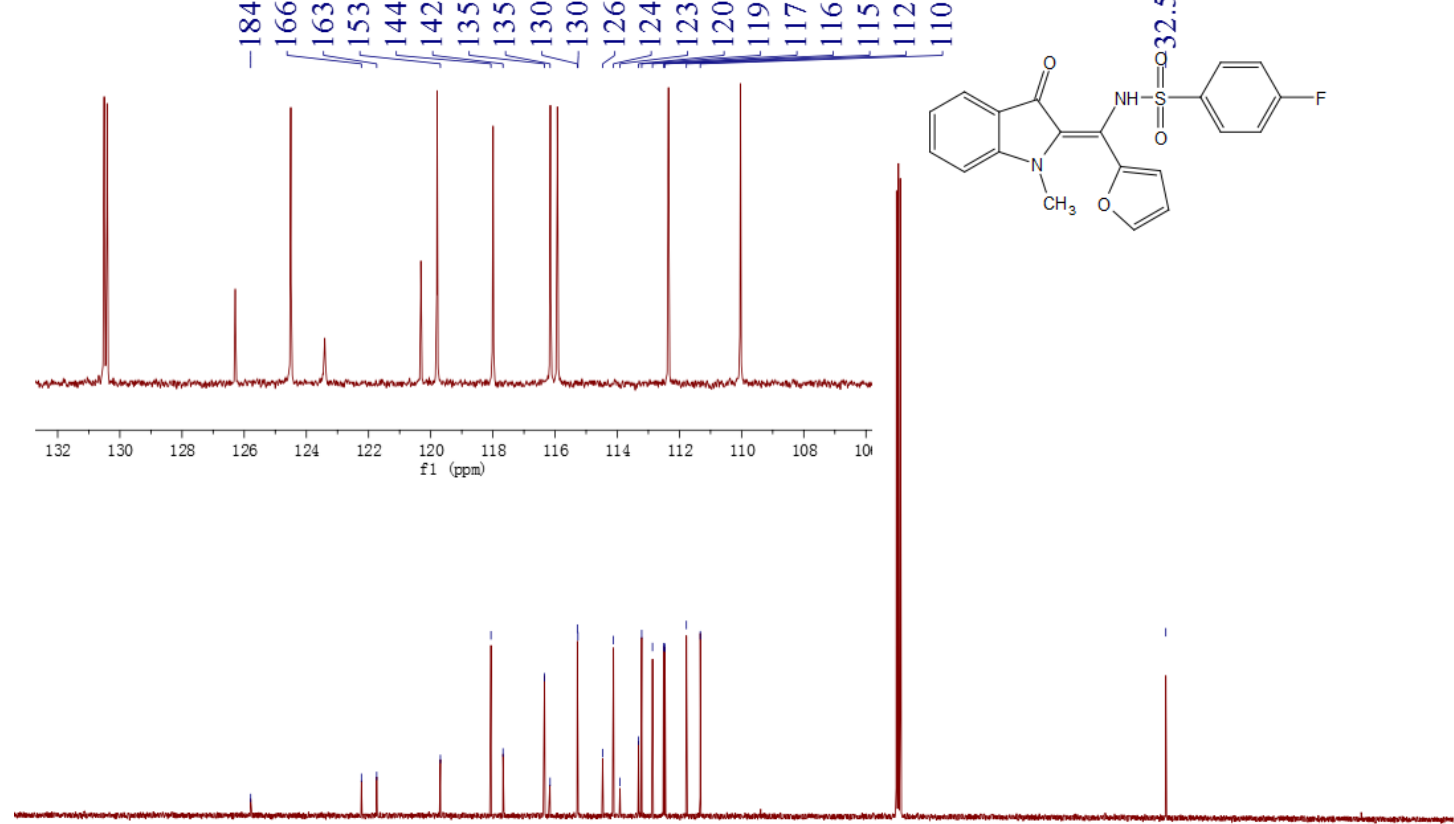

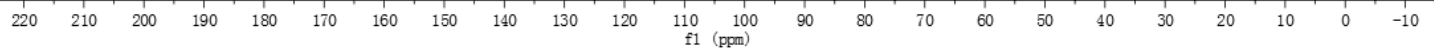

${ }^{13} \mathrm{CNMR}$ Spectrum for $\mathbf{3 A}\left(\mathrm{CDCl}_{3}, 100 \mathrm{MHz}\right)$ 


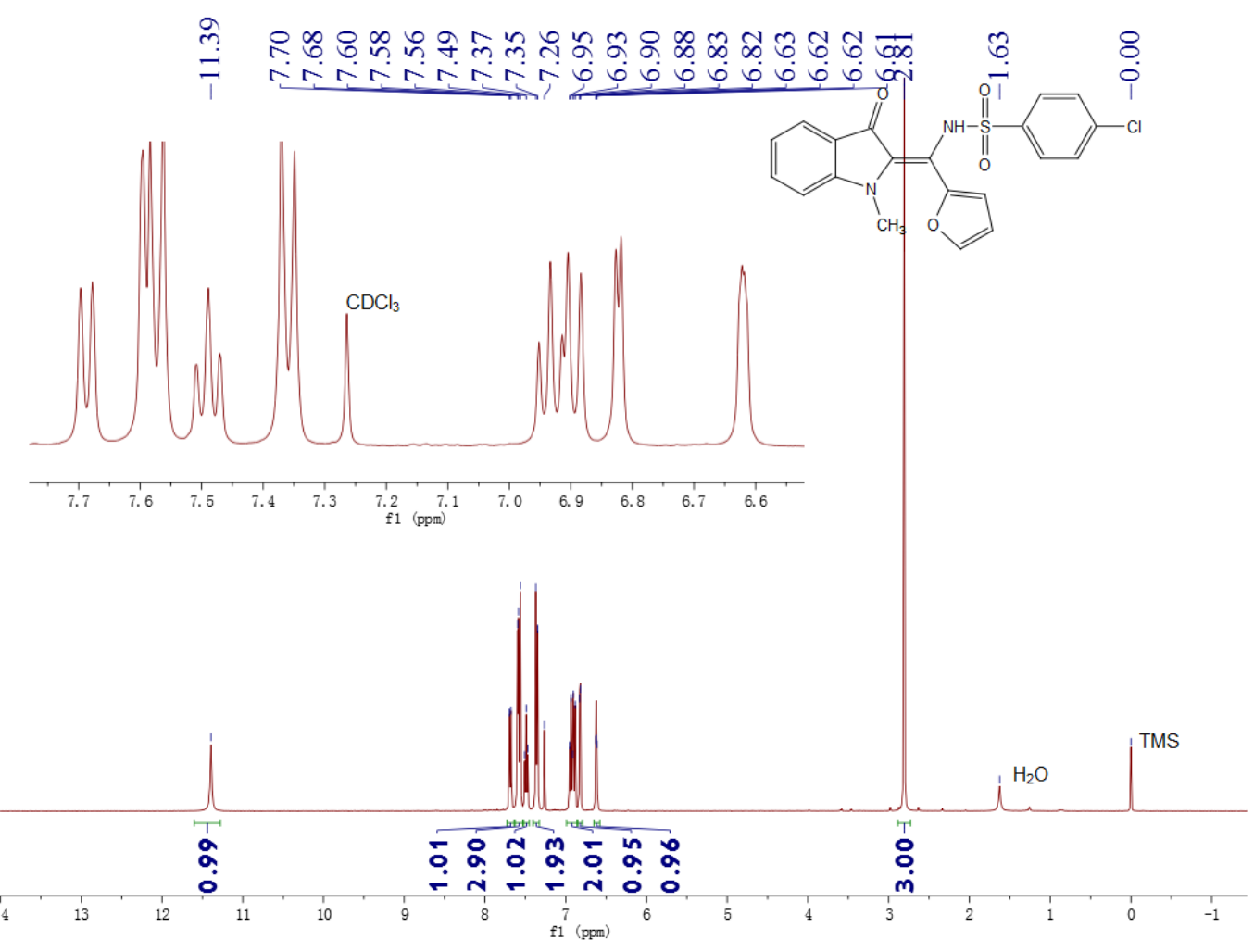

${ }^{1} \mathrm{H}$ NMR Spectrum for $3 \mathbf{B}\left(\mathrm{CDCl}_{3}, 400 \mathrm{MHz}\right)$
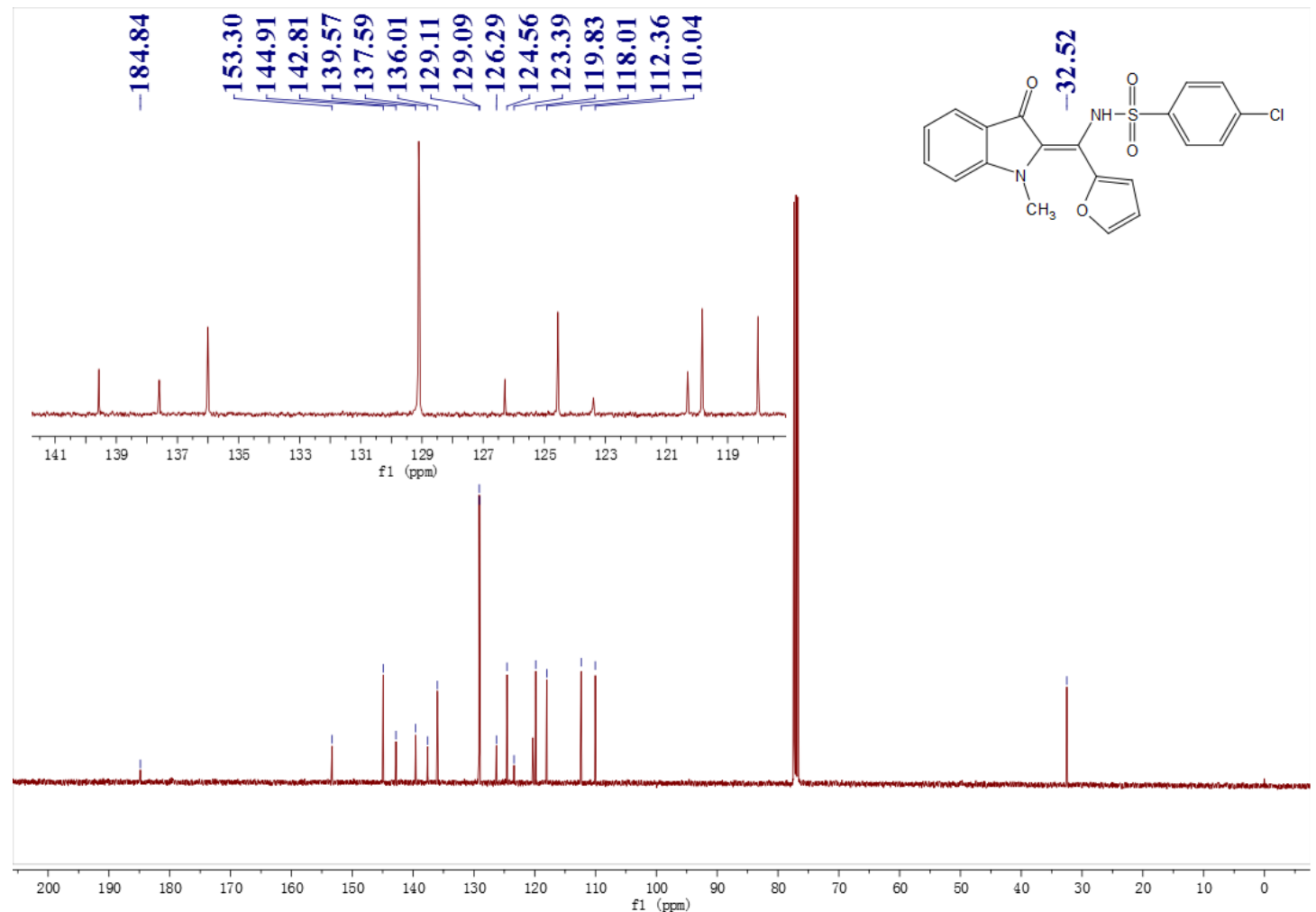

${ }^{13} \mathrm{CNMR}$ Spectrum for $\mathbf{3 B}\left(\mathrm{CDCl}_{3}, 100 \mathrm{MHz}\right)$ 


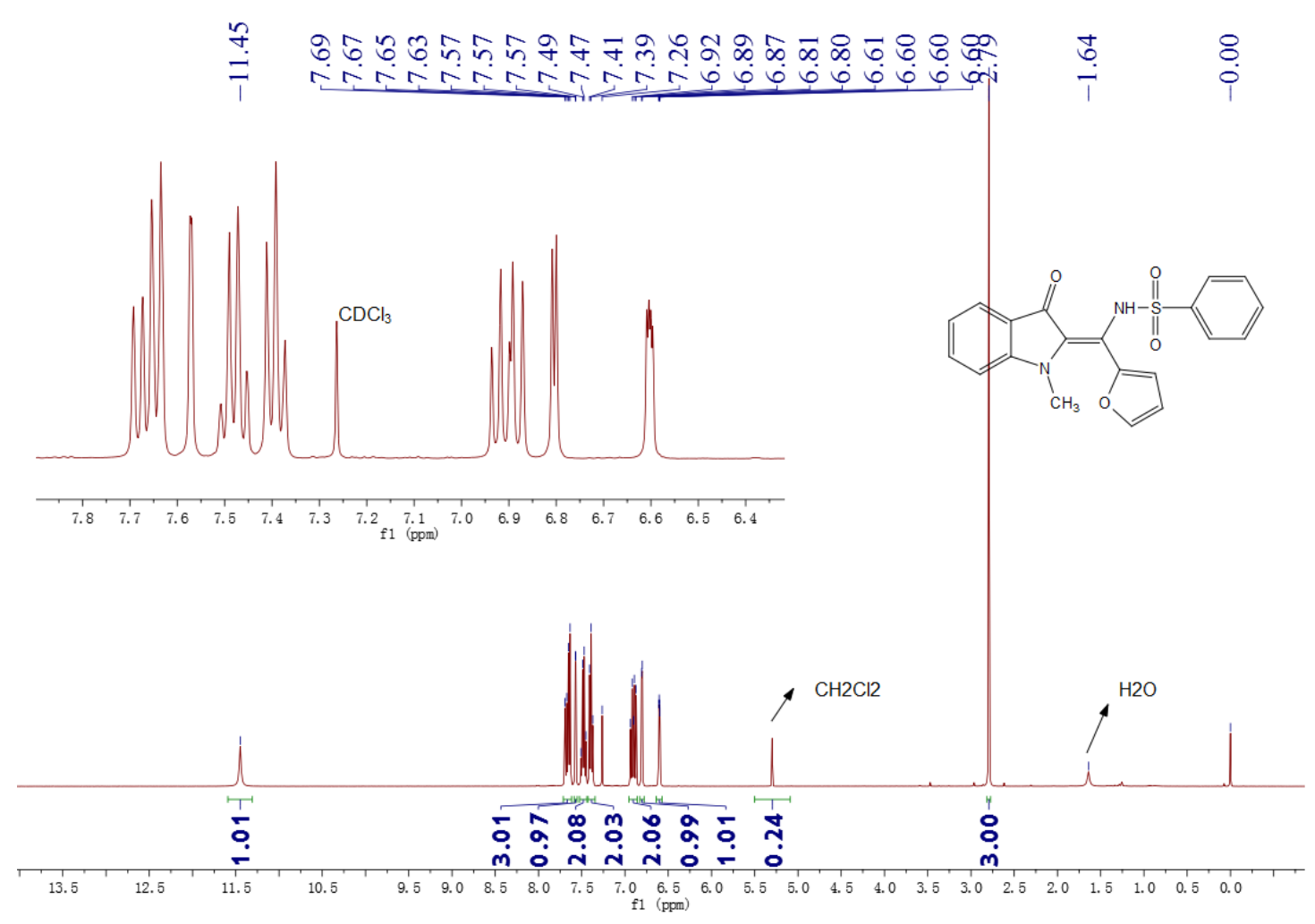

${ }^{1} \mathrm{H}$ NMR Spectrum for $3 \mathrm{C}\left(\mathrm{CDCl}_{3}, 400 \mathrm{MHz}\right)$

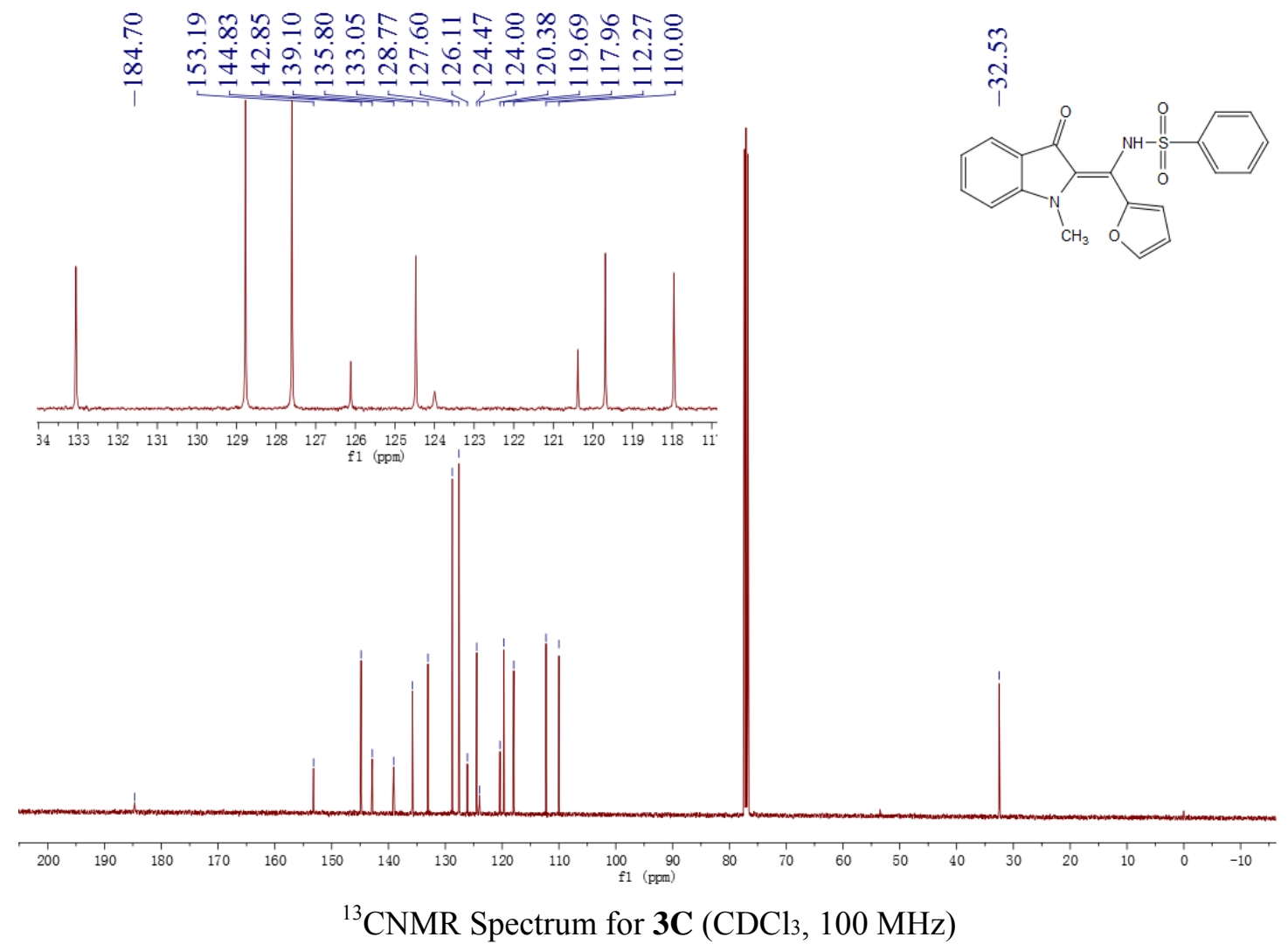




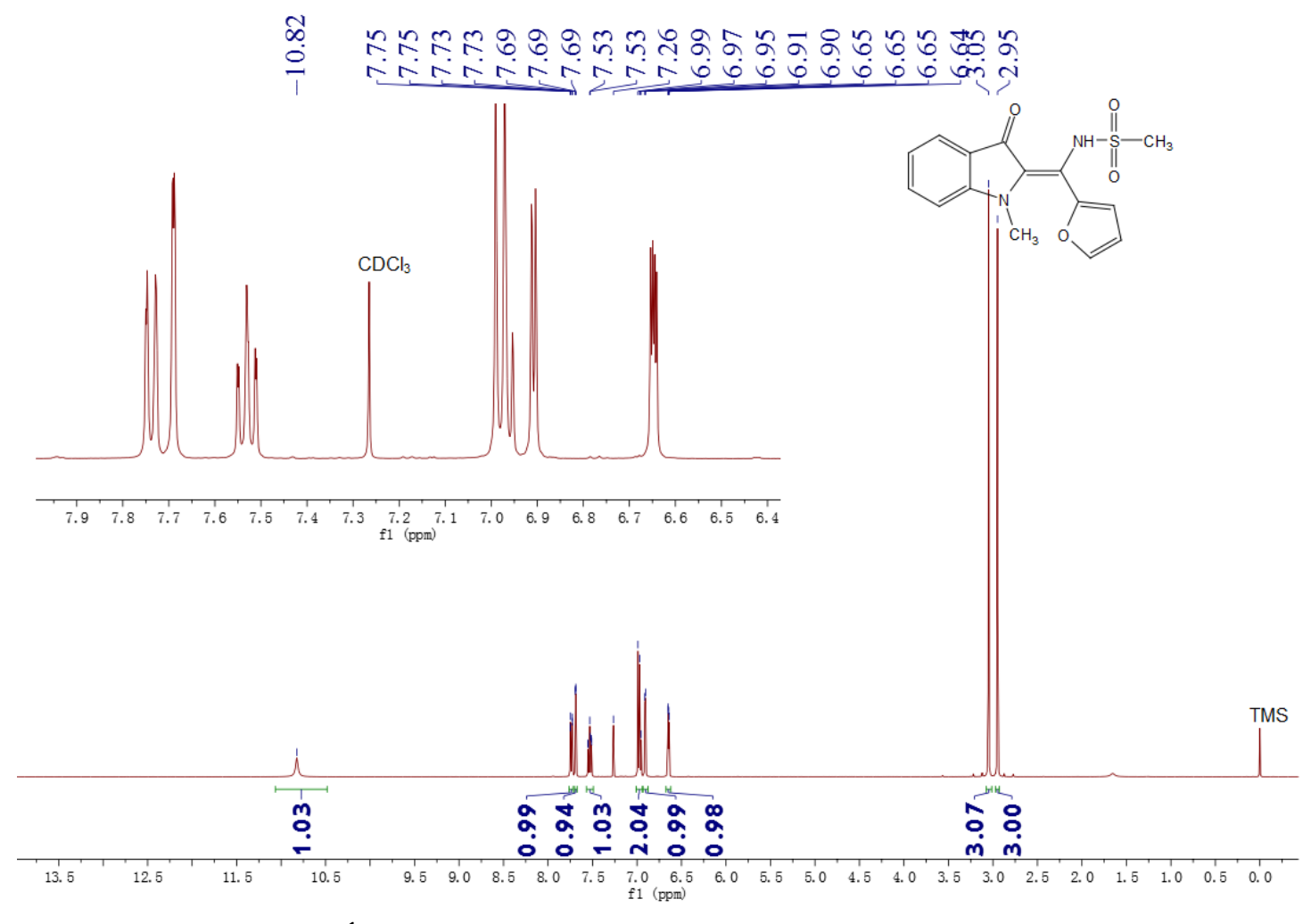

${ }^{1} \mathrm{H}$ NMR Spectrum for $3 D\left(\mathrm{CDCl}_{3}, 400 \mathrm{MHz}\right)$
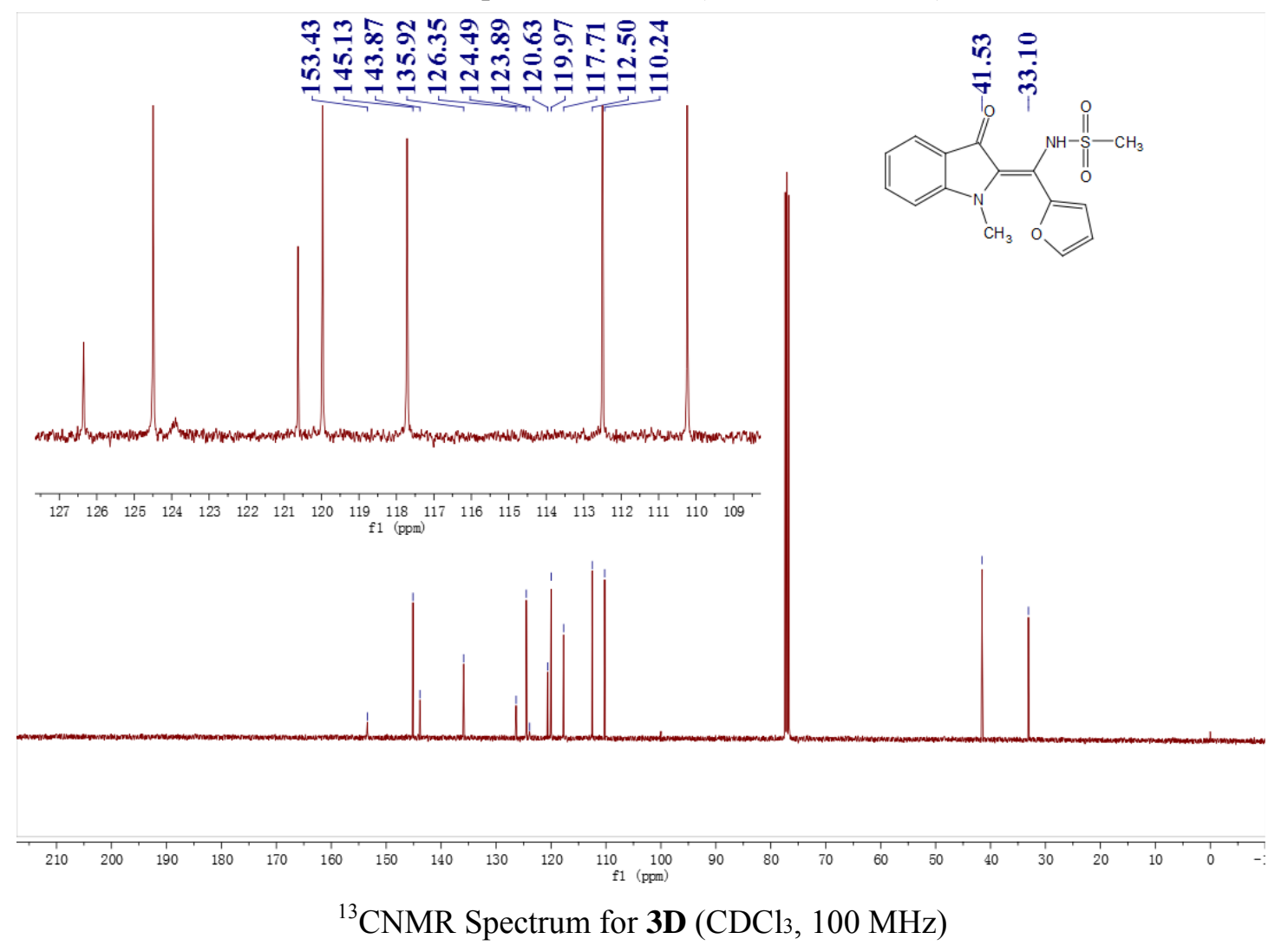


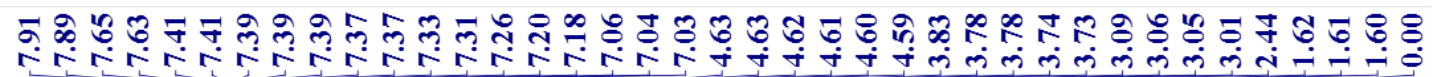
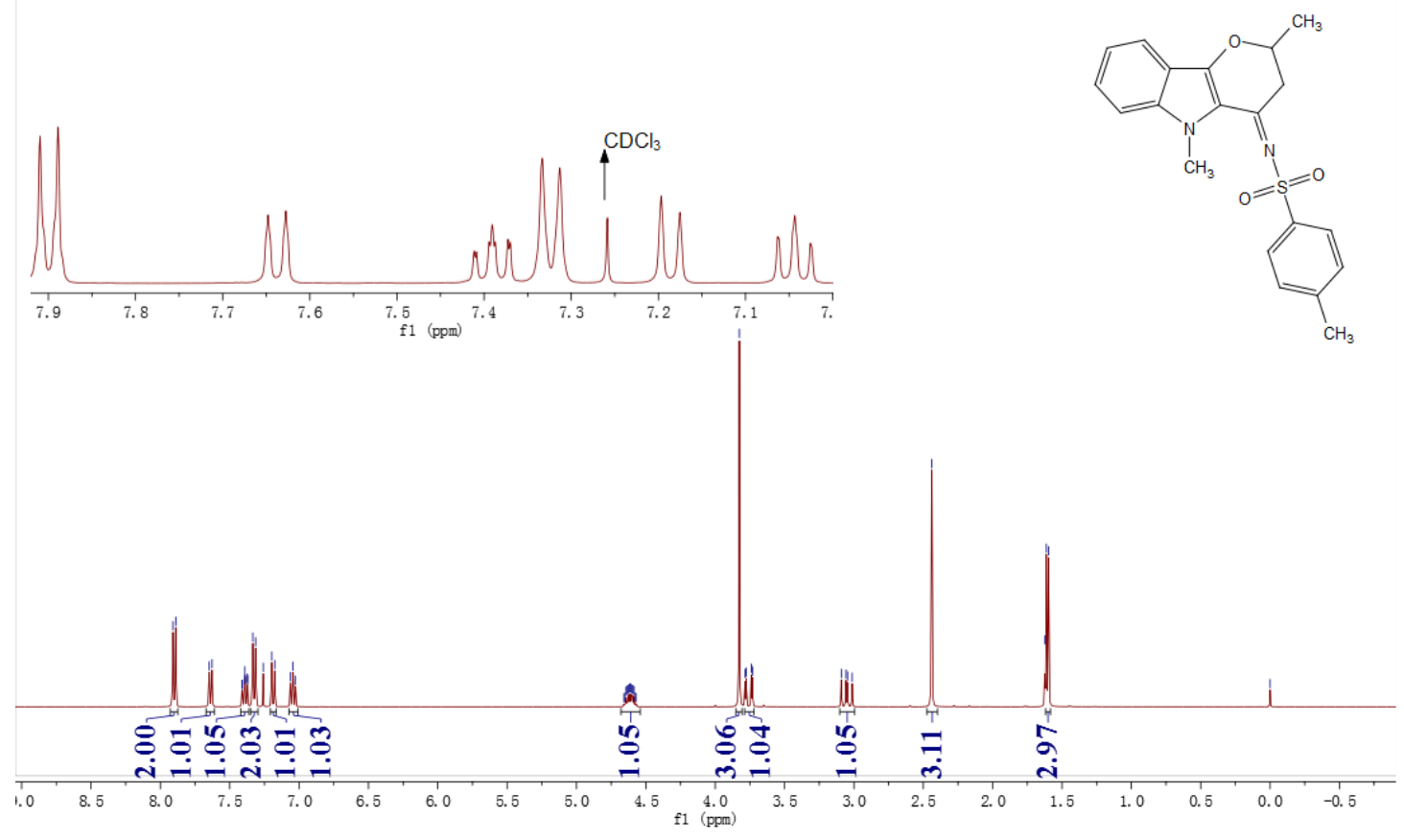

${ }^{1} \mathrm{H}$ NMR Spectrum for $\mathbf{5 a}\left(\mathrm{CDCl}_{3}, 400 \mathrm{MHz}\right)$

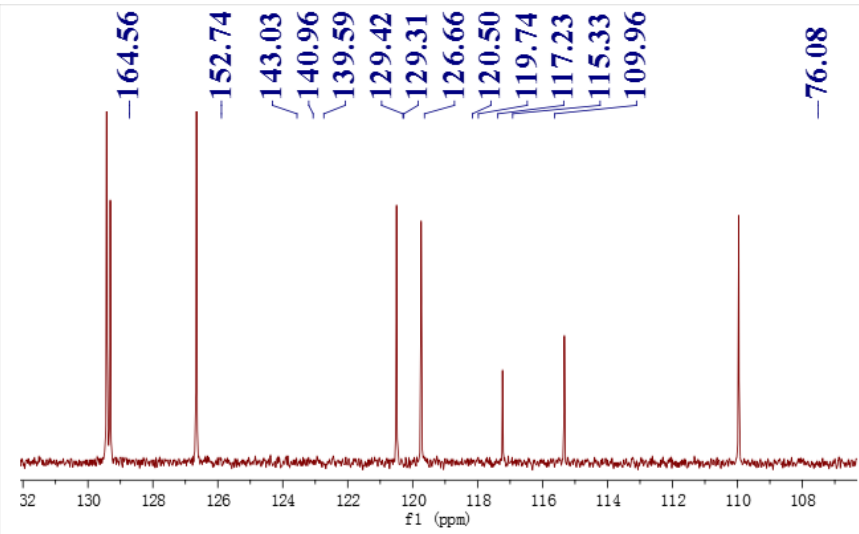

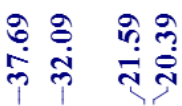
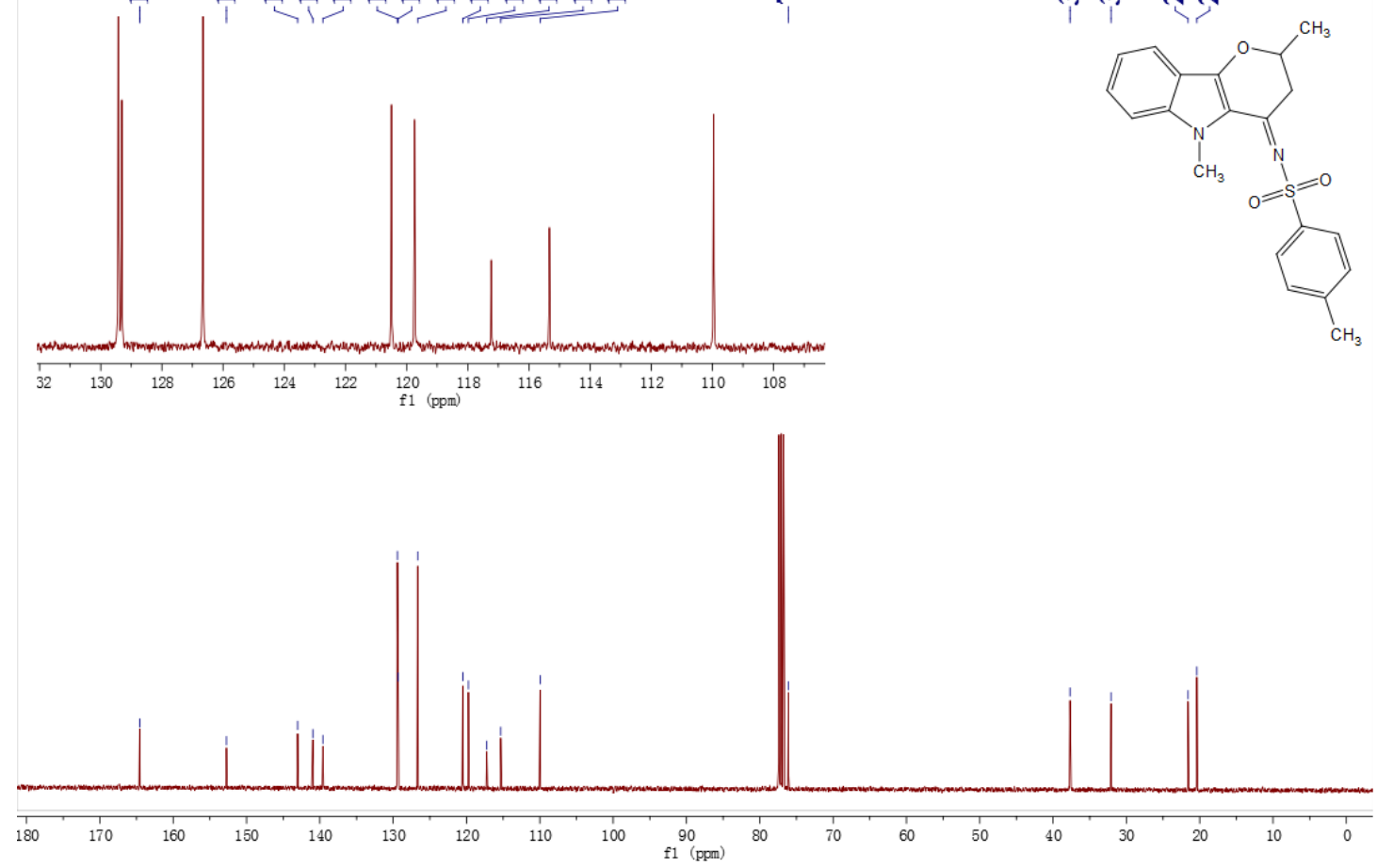

${ }^{13} \mathrm{CNMR}$ Spectrum for $\mathbf{5 a}\left(\mathrm{CDCl}_{3}, 100 \mathrm{MHz}\right)$ 


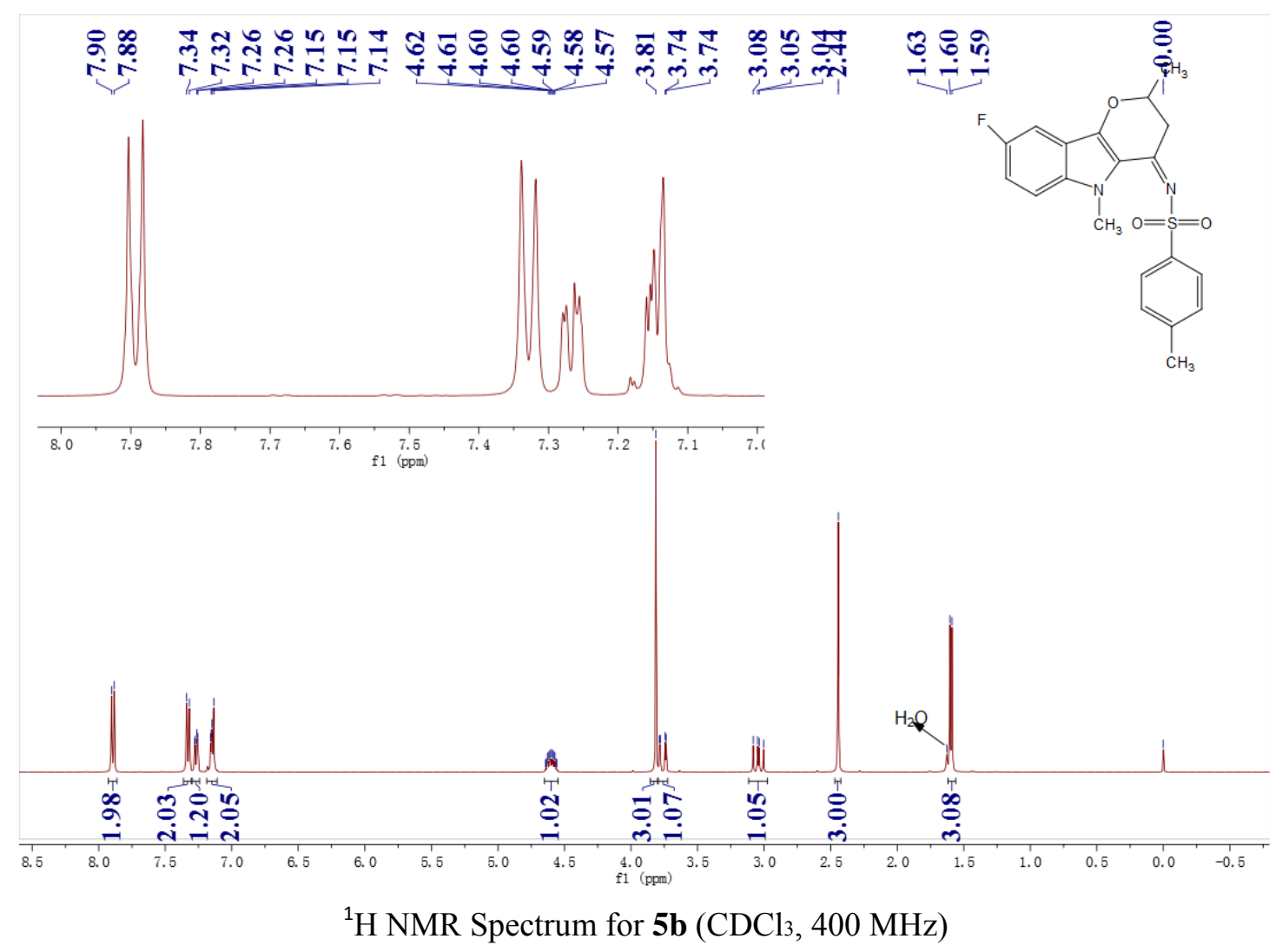

ஜ

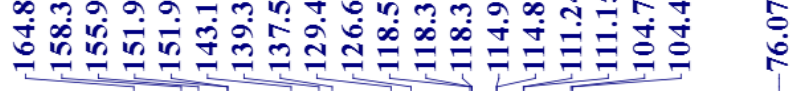

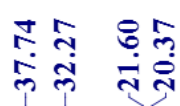
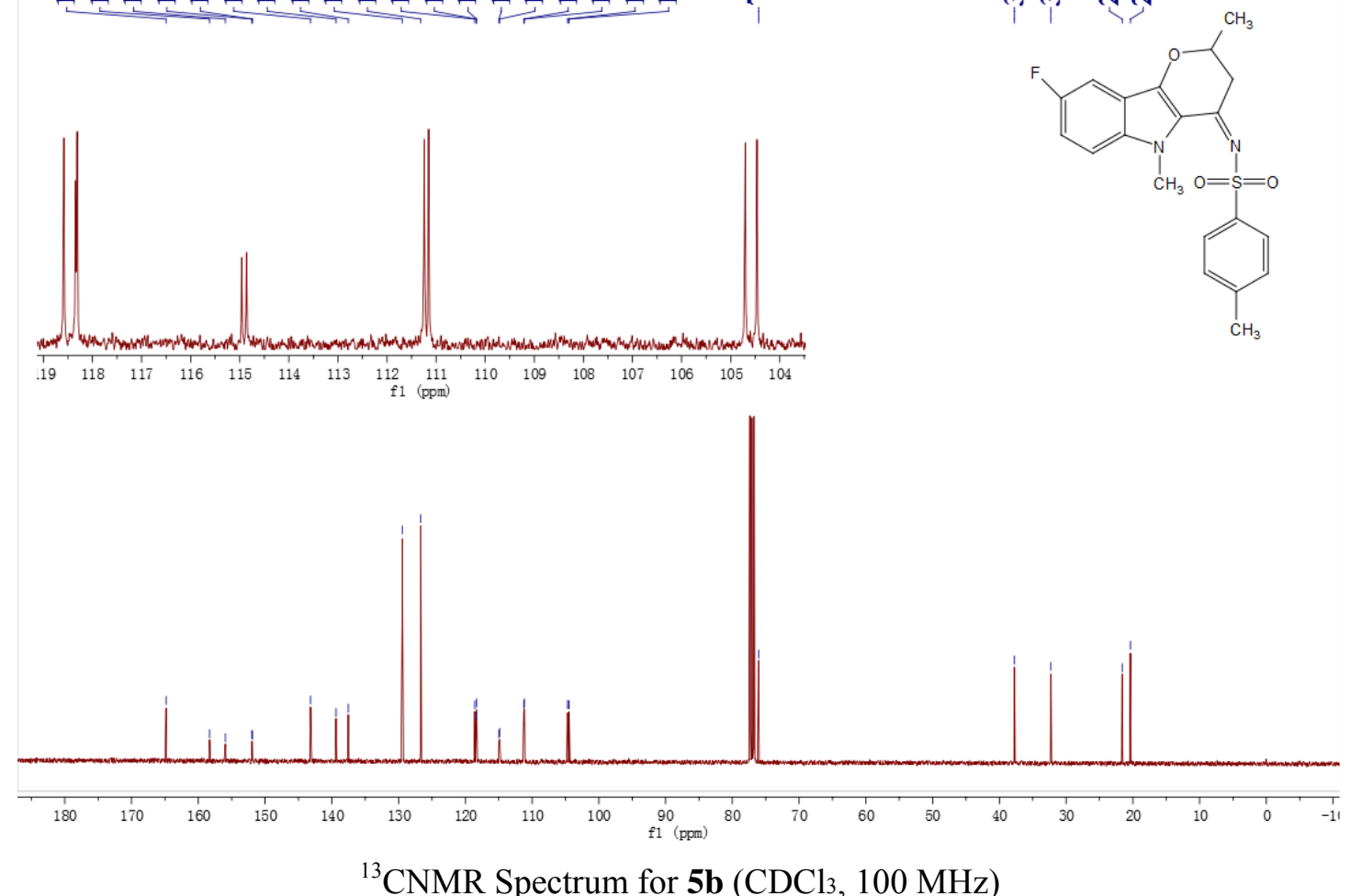

${ }^{13} \mathrm{CNMR}$ Spectrum for $\mathbf{5 b}\left(\mathrm{CDCl}_{3}, 100 \mathrm{MHz}\right)$ 


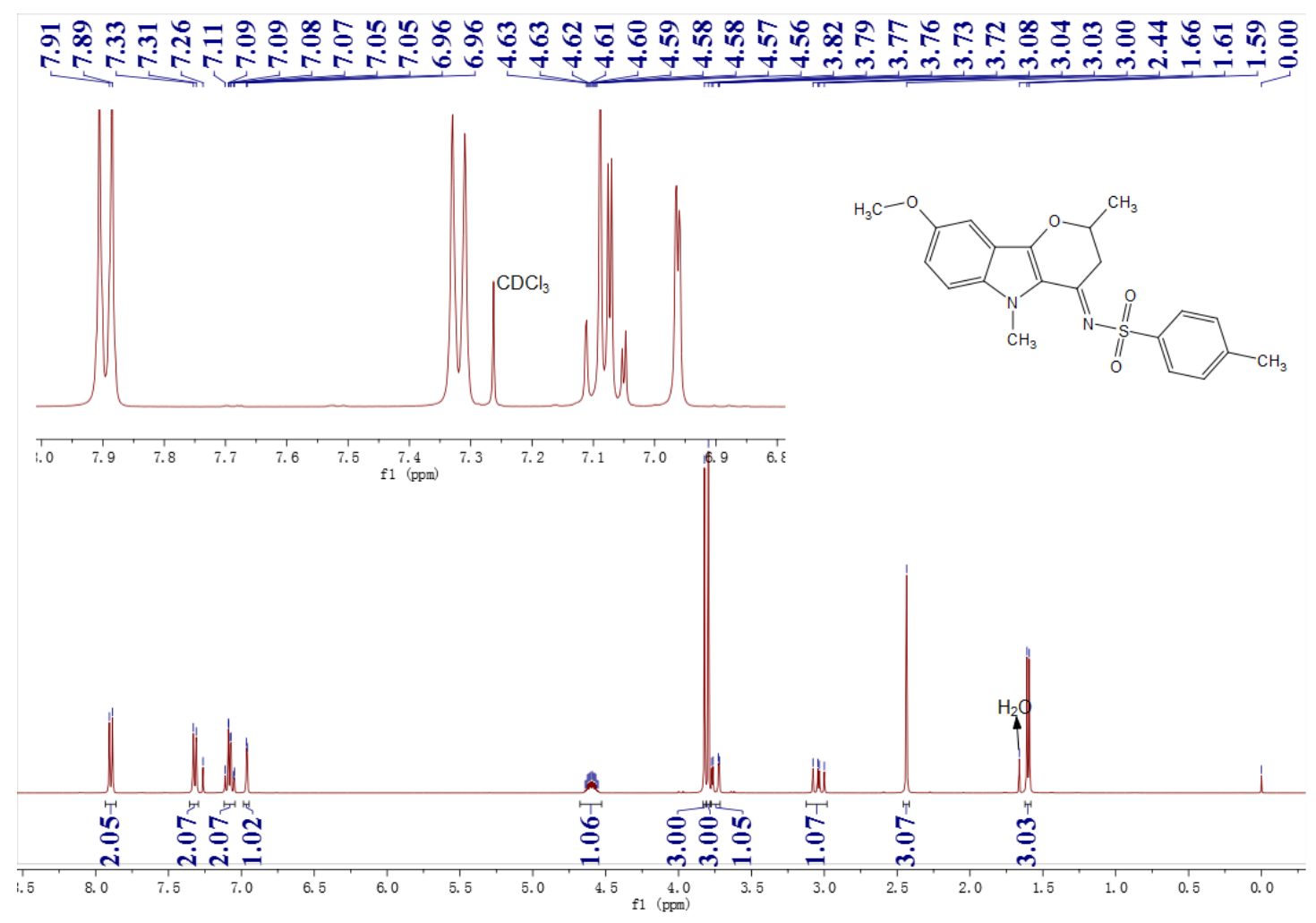

${ }^{1} \mathrm{H}$ NMR Spectrum for $5 \mathbf{c}\left(\mathrm{CDCl}_{3}, 400 \mathrm{MHz}\right)$
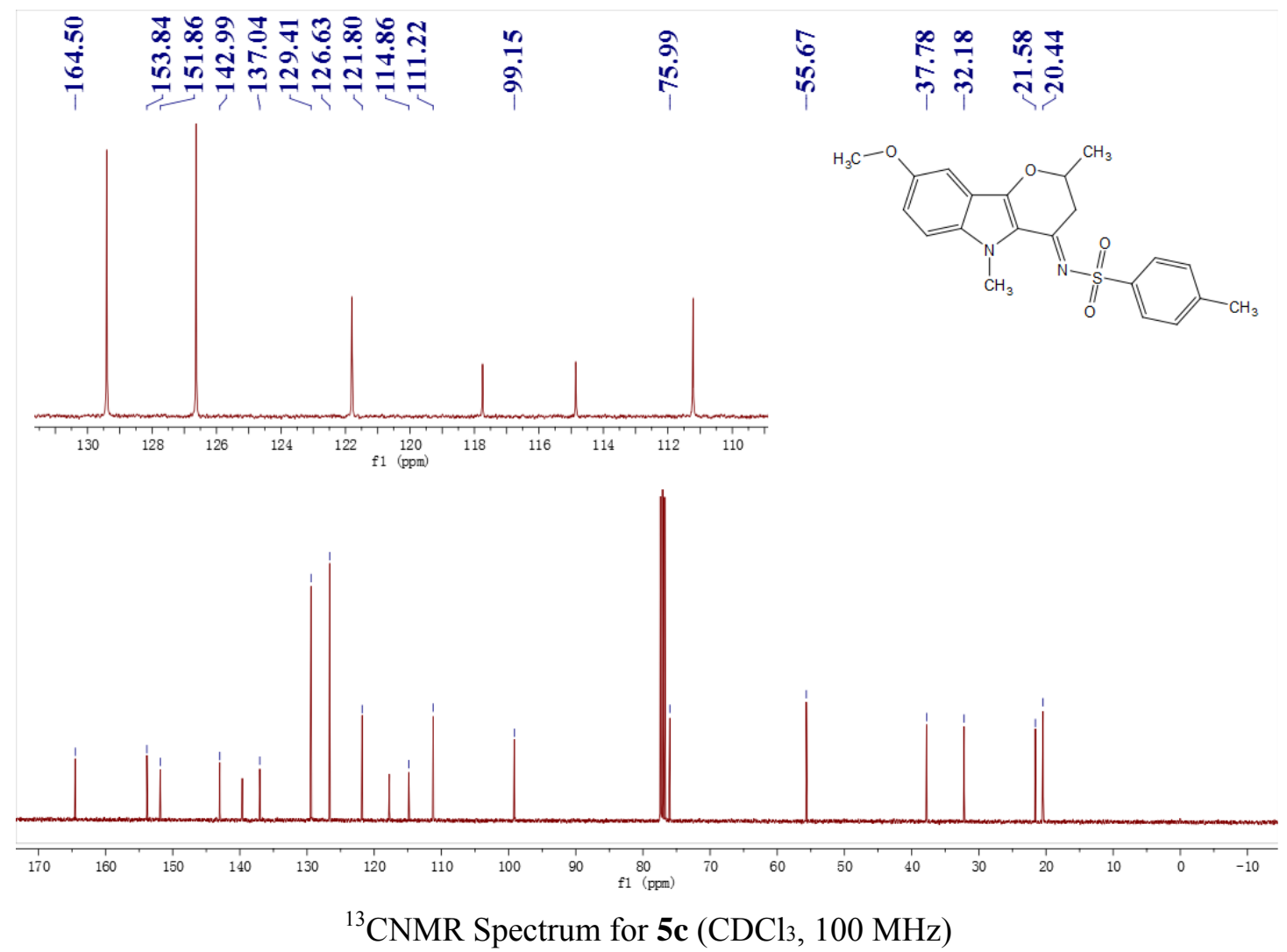


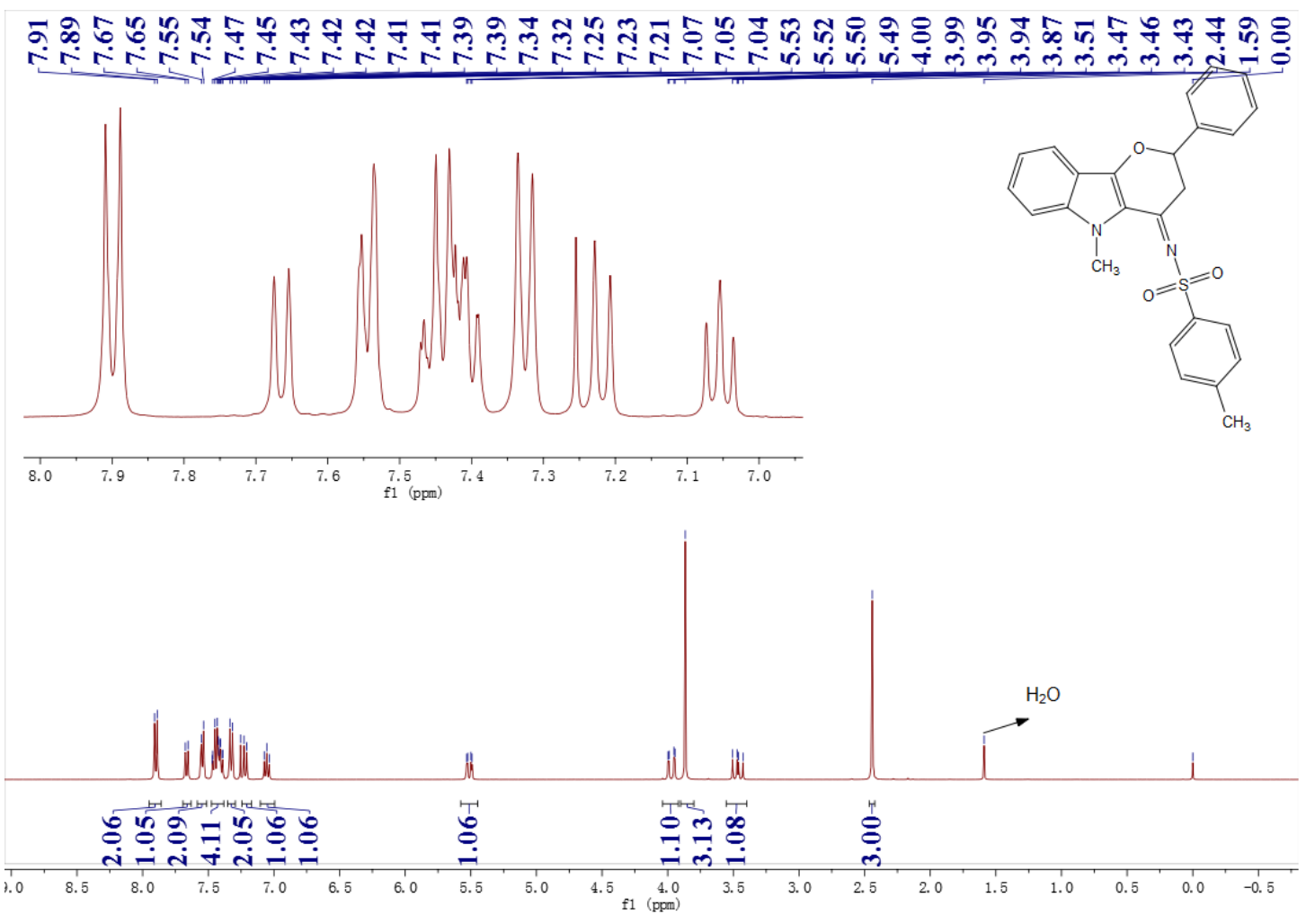

${ }^{1} \mathrm{H}$ NMR Spectrum for $5 d\left(\mathrm{CDCl}_{3}, 400 \mathrm{MHz}\right)$

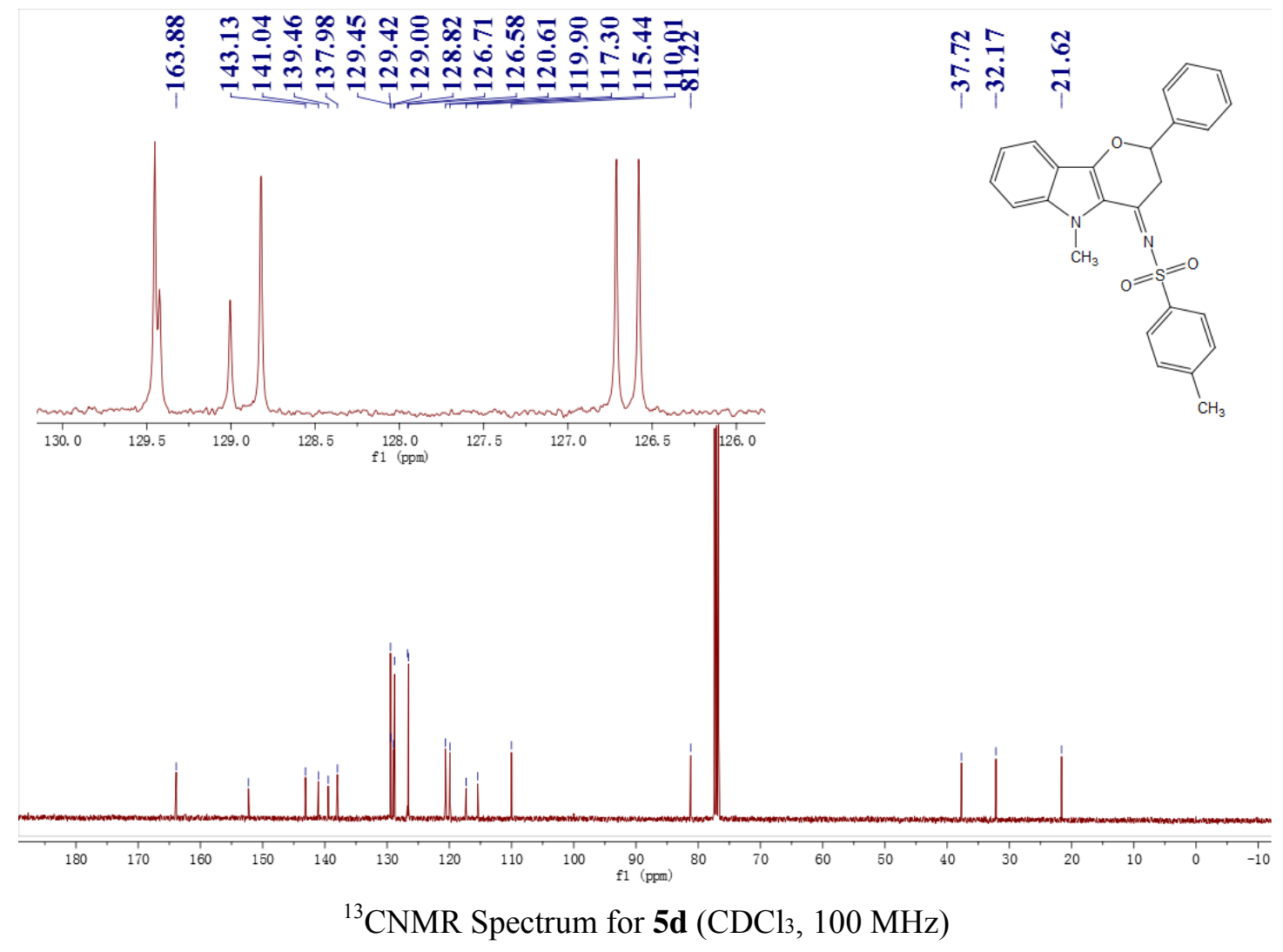




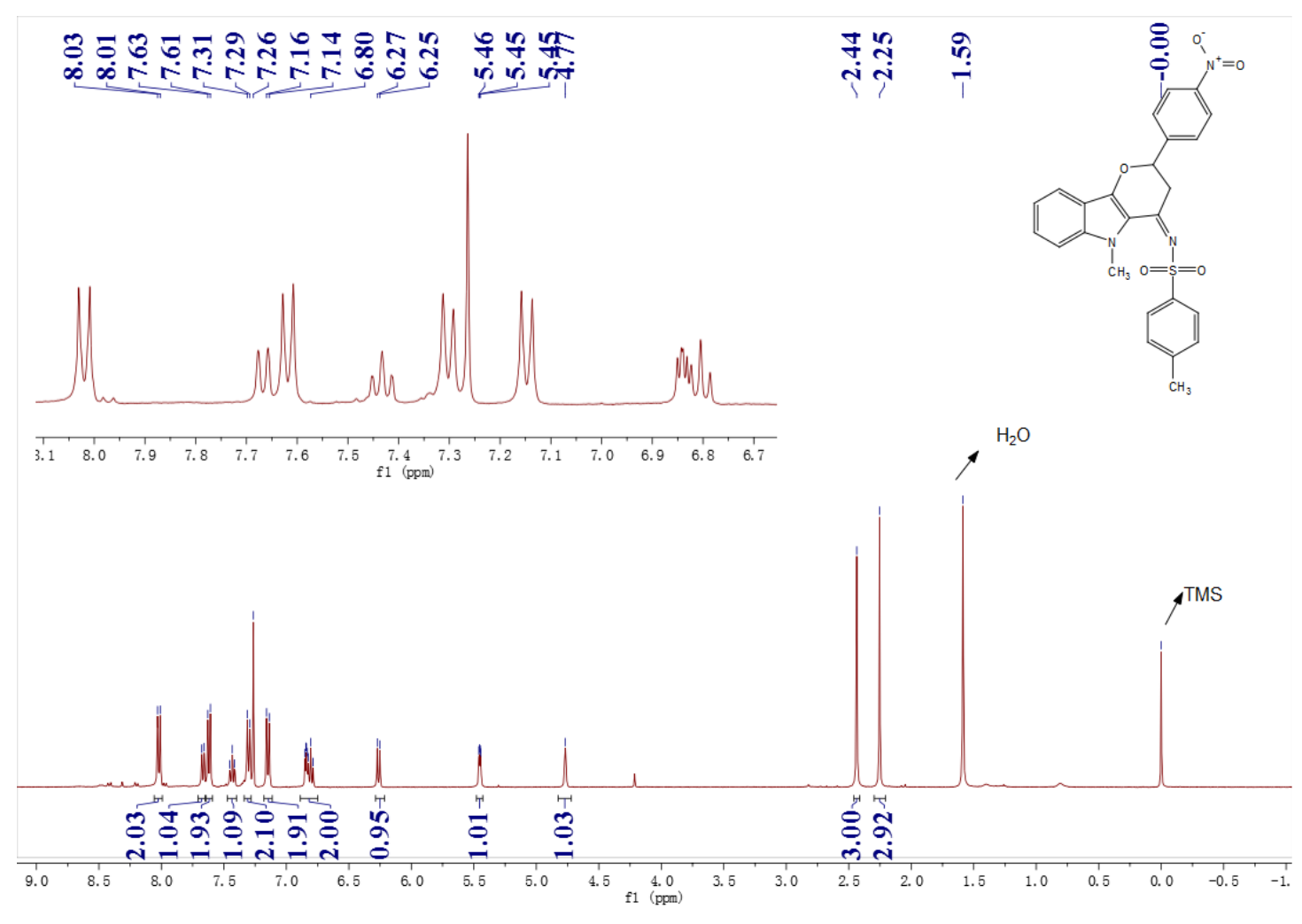

${ }^{1} \mathrm{H}$ NMR Spectrum for $5 \mathbf{e}\left(\mathrm{CDCl}_{3}, 400 \mathrm{MHz}\right)$

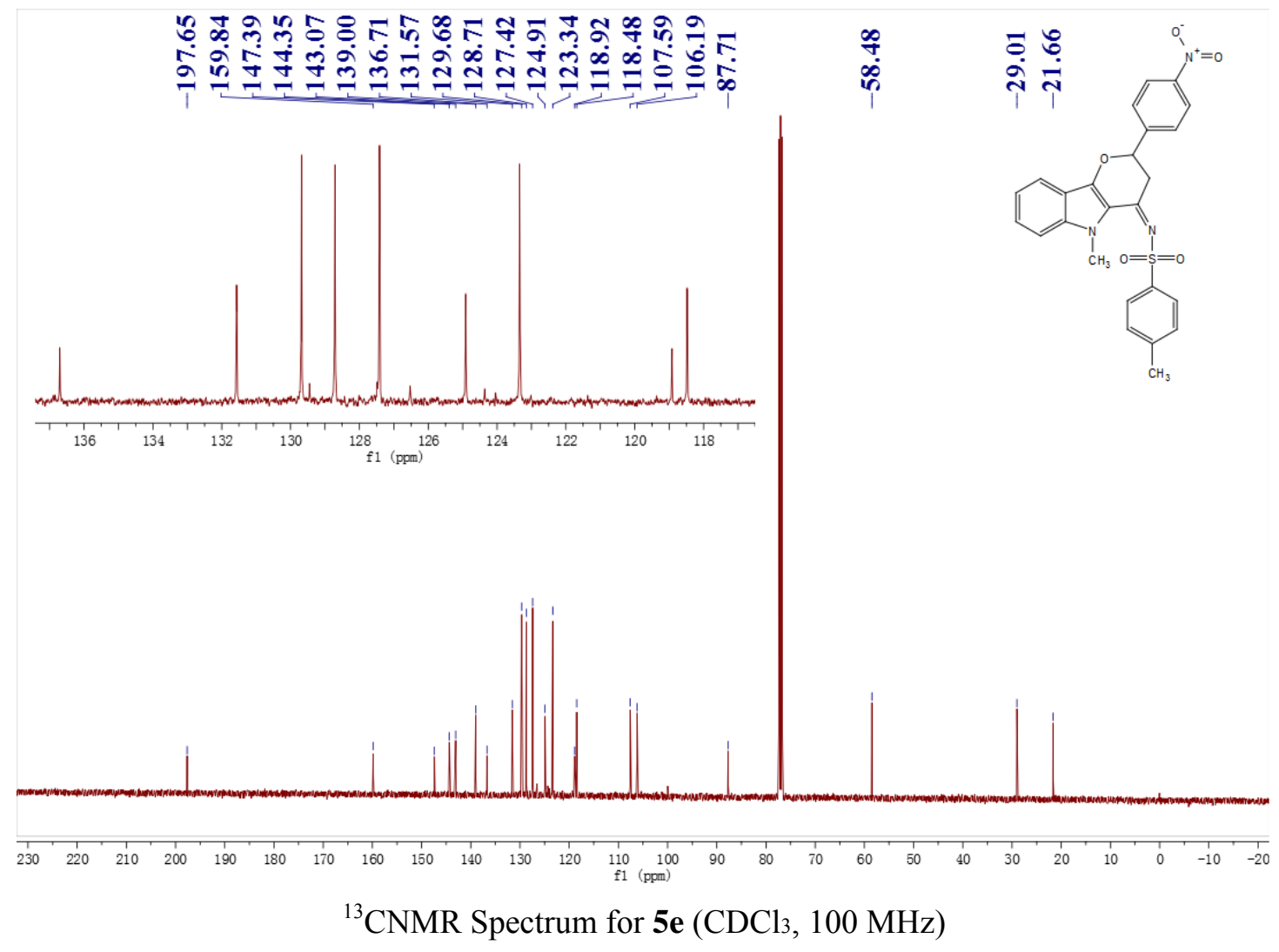




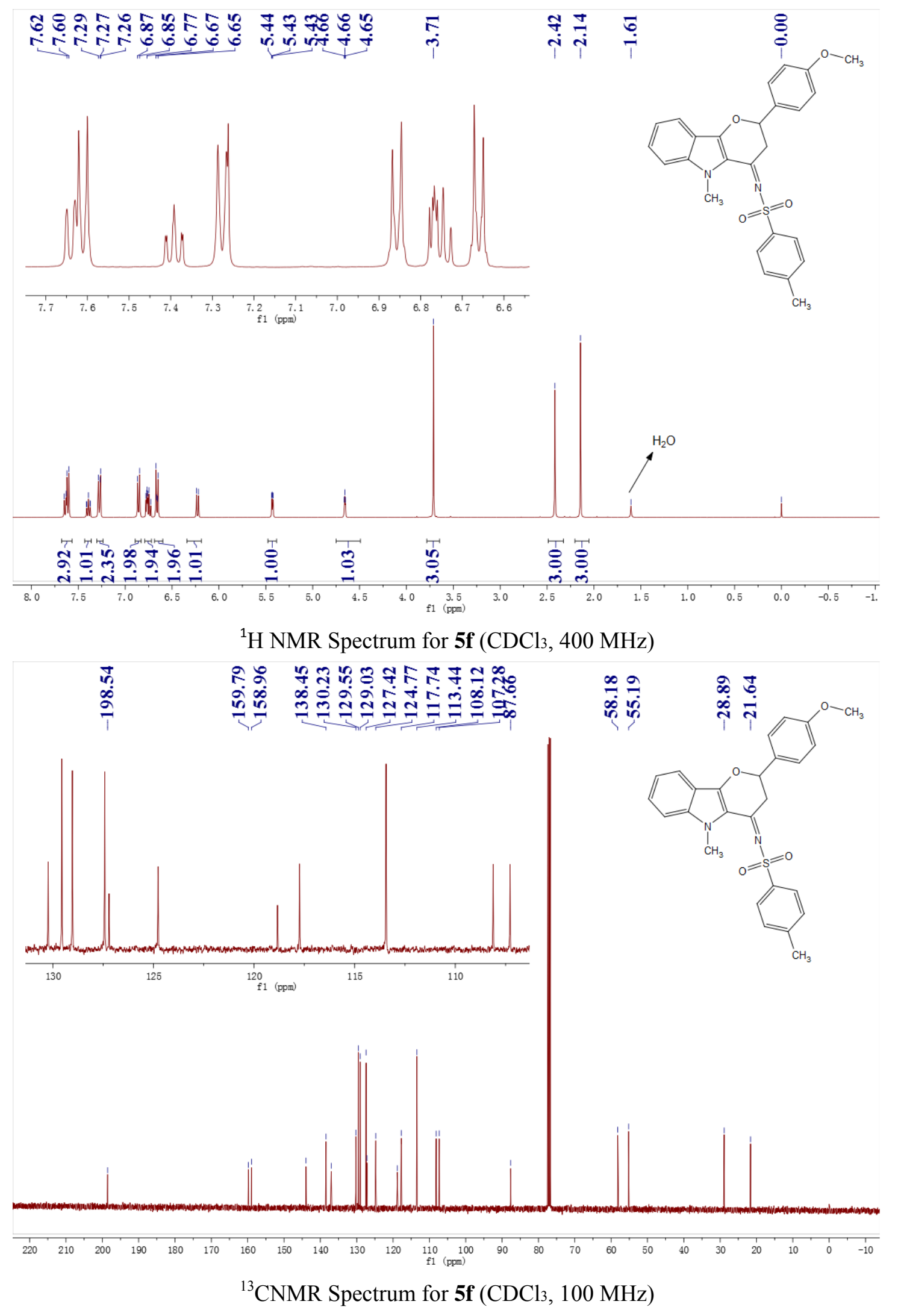



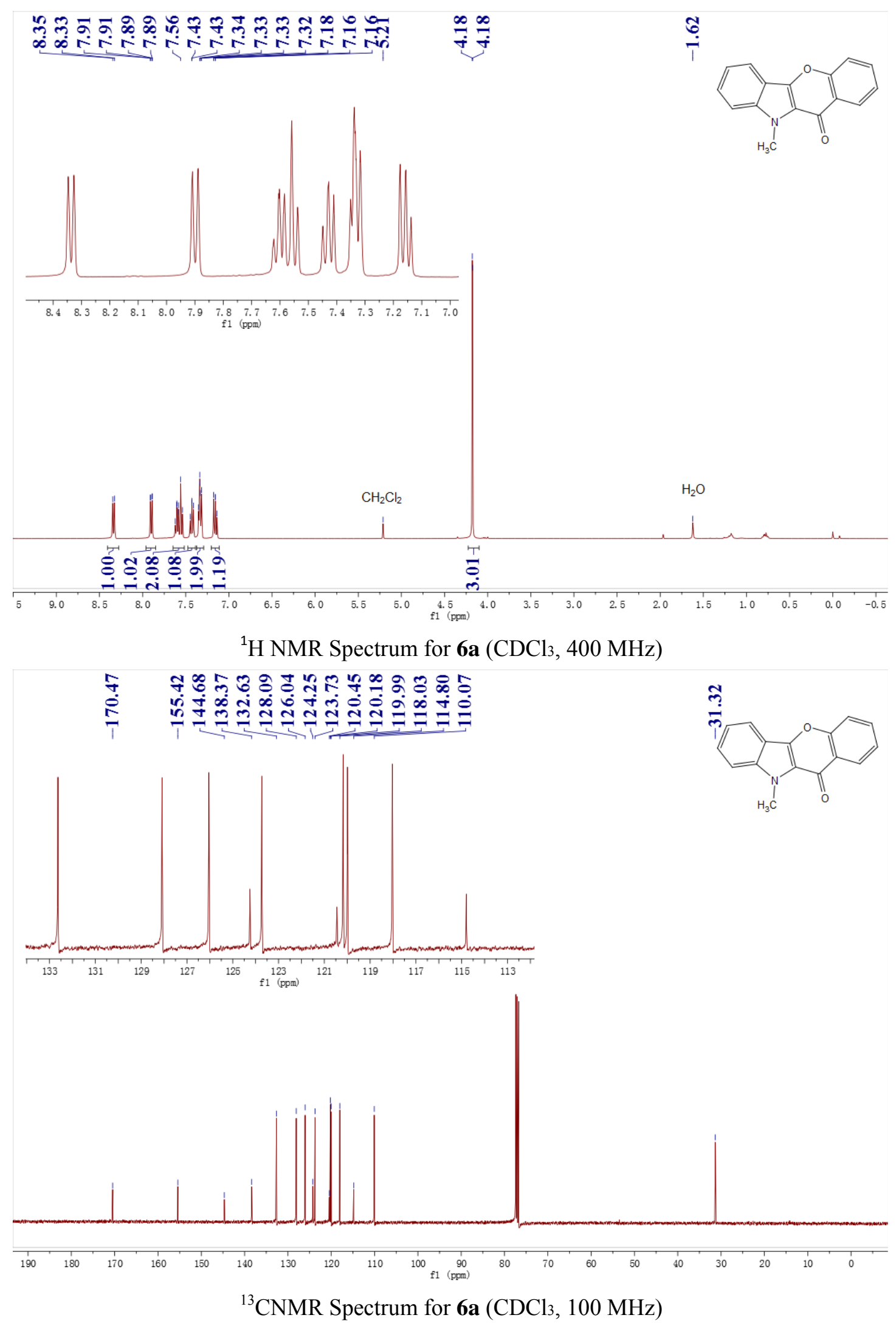


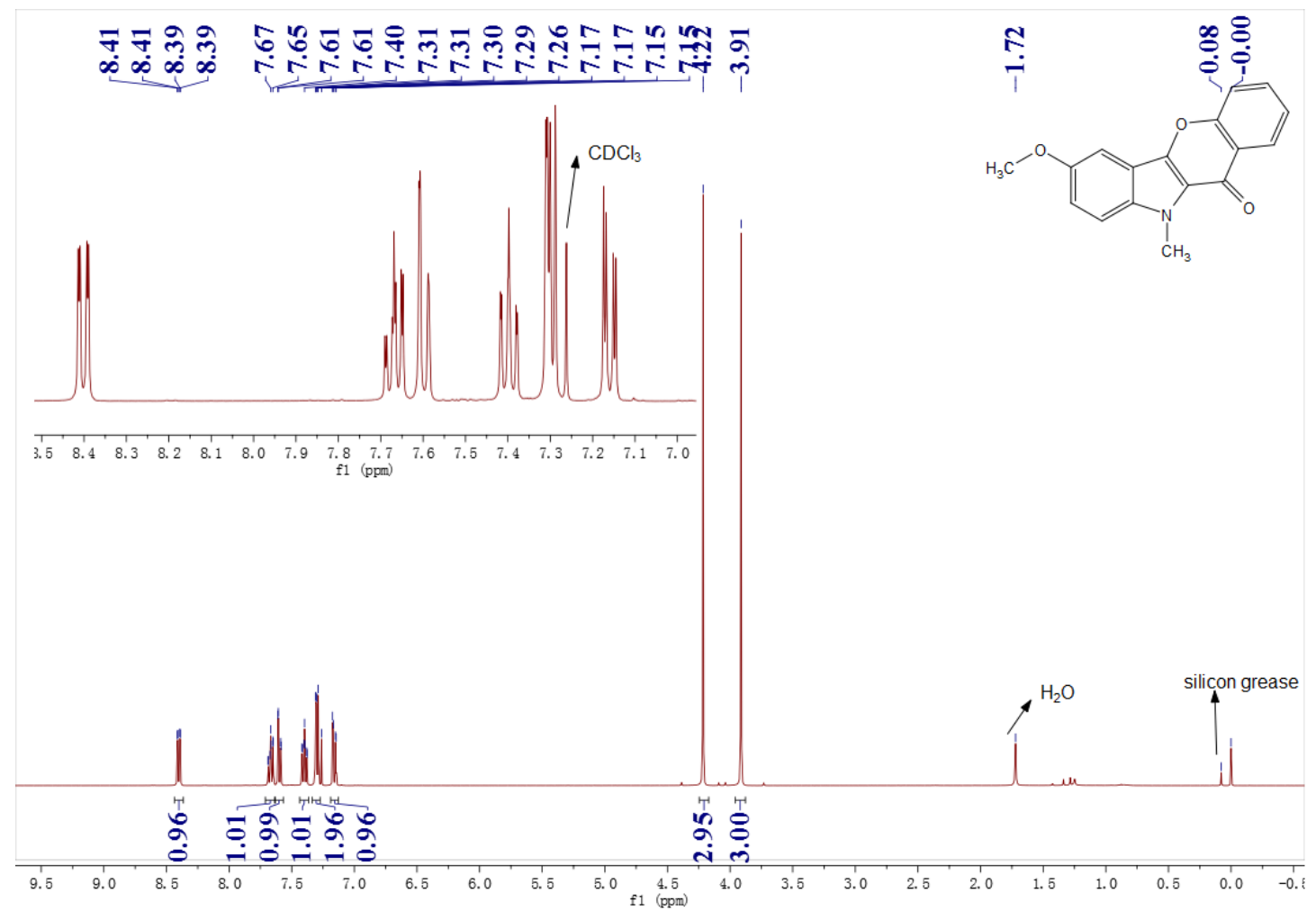

${ }^{1} \mathrm{H}$ NMR Spectrum for $\mathbf{6 b}\left(\mathrm{CDCl}_{3}, 400 \mathrm{MHz}\right)$

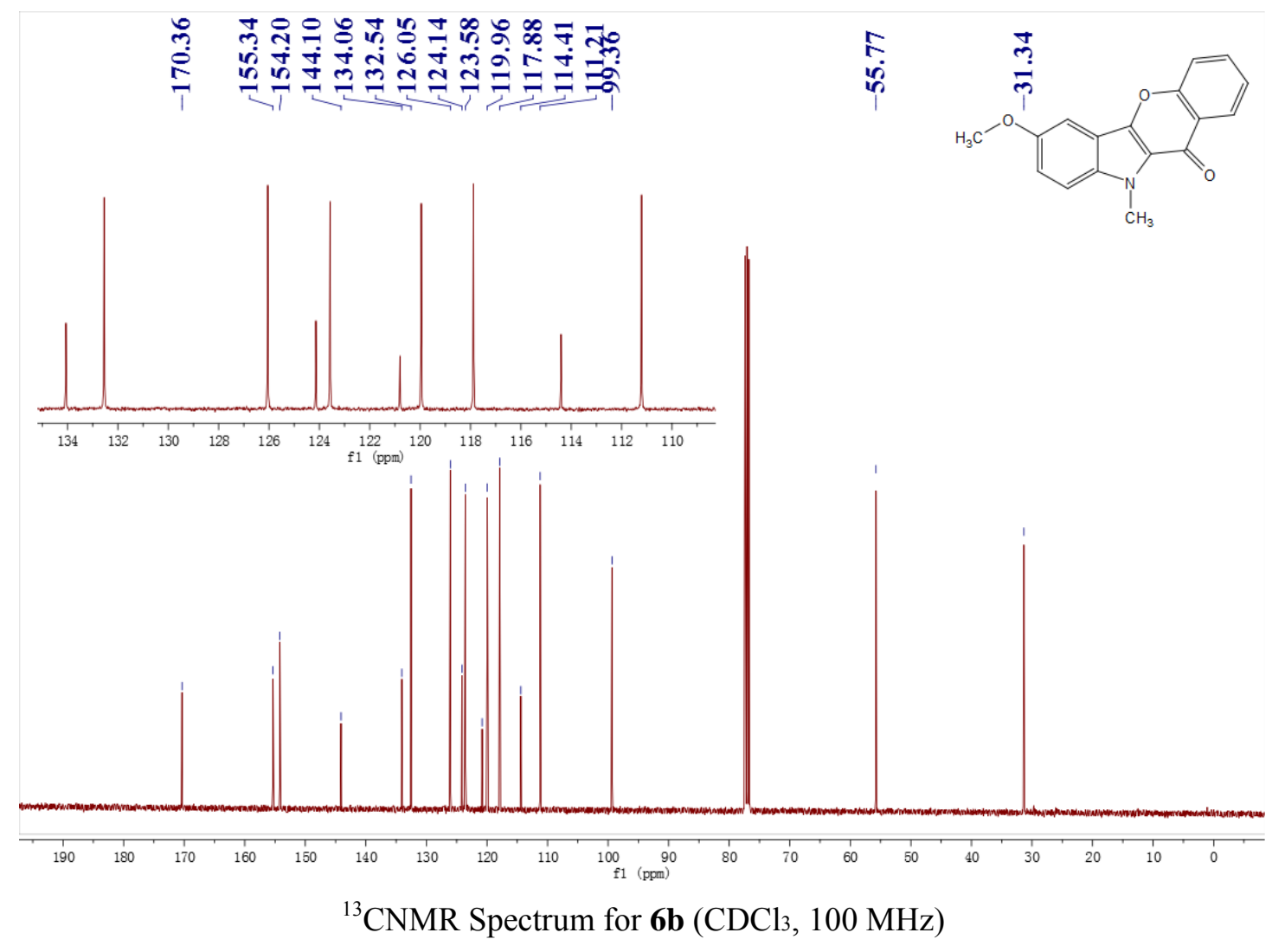




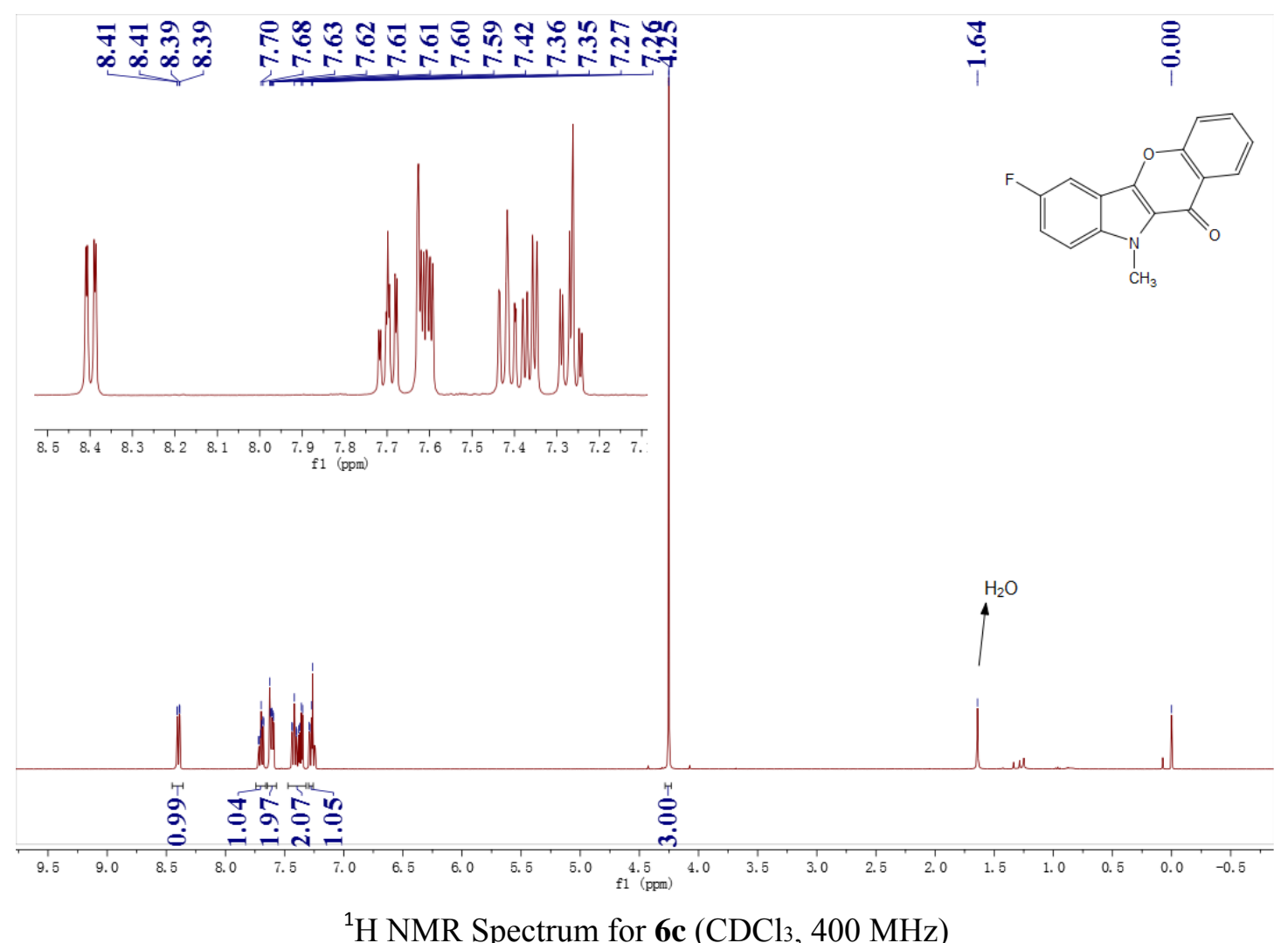

${ }^{1} \mathrm{H}$ NMR Spectrum for $\mathbf{6 c}\left(\mathrm{CDCl}_{3}, 400 \mathrm{MHz}\right)$

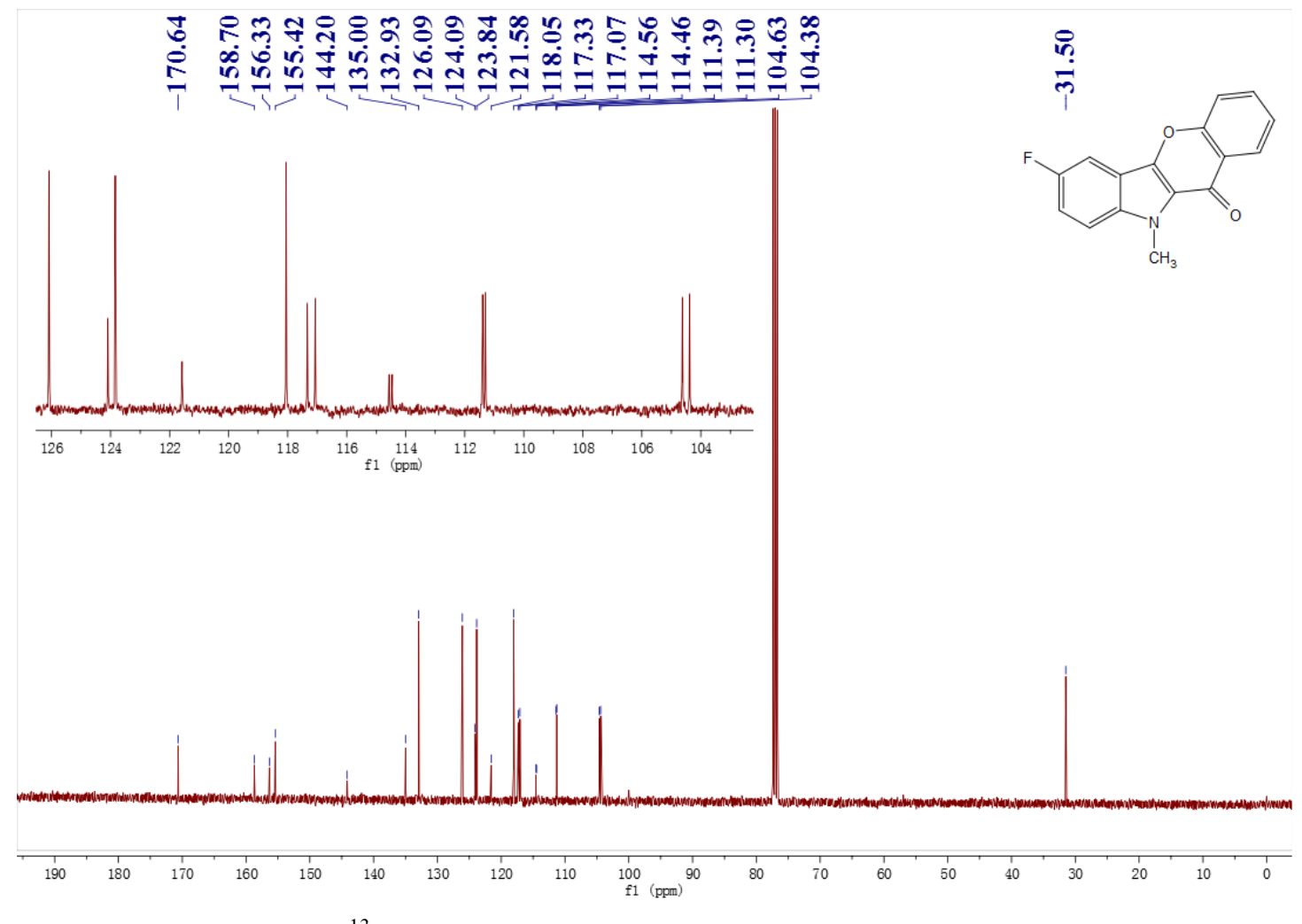

${ }^{13} \mathrm{CNMR}$ Spectrum for $\mathbf{6 c}\left(\mathrm{CDCl}_{3}, 100 \mathrm{MHz}\right)$ 